\title{
Slip Boundary Conditions for the Compressible Navier-Stokes Equations
}

\author{
Kazuo Aoki ${ }^{1,2}$, Céline Baranger ${ }^{3}$, \\ Masanari Hattori ${ }^{4}$, Shingo Kosuge ${ }^{5}$, \\ Giorgio Martalò $^{6,7}$, Julien Mathiaud ${ }^{3}$, \\ Luc Mieussens ${ }^{6,7}$ \\ ${ }^{1}$ Mathematics Division, National Center for Theoretical Sciences, National Taiwan University, \\ Taipei, Taiwan \\ kazuo.aoki.22v@st.kyoto-u.ac.jp \\ 2 Department of Mathematics, National Cheng Kung University, Tainan, Taiwan \\ ${ }^{3}$ CEA-CESTA, 15 avenue des Sablières, CS 60001, 33116 Le Barp Cedex, France \\ ${ }^{4}$ Department of Mechanical Engineering and Science, Graduate School of Engineering, Kyoto \\ University, Kyoto 615-8540, Japan \\ ${ }^{5}$ Center for Global Leadership Engineering Education, Graduate School of Engineering, Kyoto \\ University, Kyoto 615-8540, Japan \\ ${ }^{6}$ Université de Bordeaux, CNRS, Bordeaux INP IMB, UMR 5251, F-33400 Talence, France \\ ${ }^{7}$ Inria Bordeaux-Sud-Ouest, F-33405 Talence, France
}

\begin{abstract}
The slip boundary conditions for the compressible Navier-Stokes equations are derived systematically from the Boltzmann equation on the basis of the ChapmanEnskog solution of the Boltzmann equation and the analysis of the Knudsen layer adjacent to the boundary. The resulting formulas of the slip boundary conditions are summarized with explicit values of the slip coefficients for hard-sphere molecules as well as the Bhatnagar-Gross-Krook (BGK) model. These formulas, which can be applied to specific problems immediately, help to prevent the use of often used slip boundary conditions that are either incorrect or without theoretical basis.
\end{abstract}

Keywords. Boltzmann equation, Knudsen layer, Navier-Stokes equations, Slip boundary conditions, Moving boundary problems

\section{Introduction}

Gases in low-density circumstances and in microscales deviate from the local equilibrium state, so that the behavior of the gases cannot be described by the ordinary macroscopic fluid or gas dynamics. For such gases, one should use kinetic theory of gases [16, 23, 28, 14, 56, 57], whose fundamental equation is the celebrated Boltzmann equation. However, the complexity of the Boltzmann equation had hindered its application to practical gas dynamic problems. This situation was changed by the appearance and development of the direct simulation Monte Carlo (DSMC) method [8, 9]. Nowadays, in addition to this stochastic and particle method, different deterministic methods have been proposed (e.g., [11, 5, 19, 20]). Furthermore, the classical model 
Boltzmann equations, such as the Bhatnagar-Gross-Krook (BGK) [7, 68], ellipsoidalstatistical (ES) [27], and Shakhov models [42], have been continuously used and have shown their usefulness with new sophisticated numerical techniques [34, 66, 41]. Nevertheless, it is still true that the computational load for the Boltzmann equation and its models is much heavier than that for the macroscopic fluid-dynamic equations.

The measure of the deviation from the local equilibrium state is given by the Knudsen number, which is the ratio of the mean free path of the gas molecules to the characteristic length of the system. When the Knudsen number is small, the system is close to the local equilibrium. It is a common understanding that in this case, the Navier-Stokes equations, supplemented by the slip boundary conditions, provide the correct overall solution to the Boltzmann equation.

For time-independent problems, this problem had been investigated by Y. Sone since 1960's by systematic asymptotic analysis of the Boltzmann equation, and the complete slip-flow theory, which may be called the generalized slip-flow theory, has been established [53, 54, 59, 55, 60,61]; the reader is referred to his two books $[56,57]$. The generalized slip-flow theory provides the appropriate combinations of the fluid-dynamic-type equations, their boundary conditions of slip or jump type, and the kinetic corrections near the boundary (i.e., inside the so-called Knudsen layer) depending on the physical situations. It may be classified as (i) the linear theory for small Reynolds numbers $[53,54,55]$, (ii) the weakly nonlinear theory for finite Reynolds numbers $[54,59,55]$, (iii) the nonlinear theory for finite Reynolds numbers but large temperature and density variations [60], and (iv) the fully nonlinear theory [61]. The basic fluid-dynamic-type equations are the Stokes equations in (i), the so-called incompressible Navier-Stokes type in (ii), and the ghost-effect equations in (iii). In (iv), the overall equations are of the Euler type, but its solution needs to be matched with the solution of the equations of the viscous boundary-layer type with appropriate slip boundary conditions. The two books by Sone $[56,57]$ give the summary of all these fluid-dynamic-type systems and recipes for applications according to the physical situations under consideration. Therefore, we do not have to go back to the Boltzmann equation and can solve the problems in the framework of macroscopic gas dynamics. It should be noted that the extension of the generalized slip flow theory to time-dependent problems has been discussed in Sect. 3.7 of [57], and the extension of the linear theory has been completed recently $[65,25,26]$.

In the present study, we focus our attention on the fully nonlinear setting in which the Mach number is finite and density and temperature variations are large. This is the most general setting and corresponds to Sone's fully nonlinear theory [61]. This theory is theoretically rigorous in the sense that it is a consequence of a systematic asymptotic analysis. Since a Hilbert-type expansion is used, the overall fluid-dynamic equations are of the Euler type. Therefore, one needs to introduce the intermediate layer, i.e., the viscous boundary layer, in order to match the overall Euler-type solution with the Knudsen layer, and the slip boundary conditions are derived for the viscous boundarylayer equations. However, this structure gives less flexibility because the theory cannot be applied to the problems in which the viscous boundary layer does not appear, such as flows with boundary layer separation. In addition, since steady flows are considered in [61], the theory naturally cannot be applied to unsteady problems, in particular, those containing moving boundaries. Furthermore, even for the problems in which 
the viscous boundary layer appears on the whole boundary, the numerical solution of the system of fluid-dynamic equations is not straightforward because it requires the matching between the Euler and viscous boundary layer equations. Therefore, it is desirable to have a more flexible system composed of a single set of fluid-dynamic equations and appropriate slip boundary conditions, applicable to more general fully nonlinear and time dependent problems, as an alternative to [61]. This is the motivation of the present study.

As the appropriate single set of fluid-dynamic equations, we consider the NavierStokes equations for compressible fluids, the so-called compressible Navier-Stokes equations, since it can naturally describe the situation in which the flow field is separated into bulk inviscid flow and viscous boundary layers. Therefore, we just need the appropriate slip boundary conditions that are to be combined with the compressible Navier-Stokes equations.

The derivation of the slip boundary conditions for the compressible Navier-Stokes equations is a classical problem, and its outline can be found in many classical textbooks (e.g., [28, 14]). However, it has not been the subject of a separate paper except [18]. In fact, to the best of the authors' knowledge, it is impossible to find, in the literature, the rigorously derived formulas of the slip boundary conditions with the explicit values of the slip coefficients that can be applied immediately to specific problems (the result of [18], which is also restricted to the case of steady flows with stationary boundaries, will be discussed in Sect. 5.3.4). For this reason, slip boundary conditions that are incorrect or derived by crude arguments without rigorous analysis have been widely used. An example is that the slip boundary conditions derived in the linear or weakly nonlinear setting (i.e., for small Mach numbers) are sometimes misused for the compressible Navier-Stokes equations.

Therefore, we revisit this problem, aiming at deriving the slip boundary conditions in a precise way on the basis of the Boltzmann equation and providing the formulas for time-dependent problems with arbitrarily moving boundaries that can be used immediately. This gives a reliable and simpler tool to analyze gas flows at small Knudsen numbers, since the numerical analysis of the Boltzmann and its model equations becomes increasingly difficult as Knudsen number becomes small. We will basically follow the procedure of the analysis of the Knudsen layer developed by Sone in his papers and books cited above and exploit the existing numerical solutions for the Knudsen-layer problems. Therefore, the essence of the analysis is not new. However, it requires a careful and subtle analysis, and moreover the obtained results are new. It should also be remarked that the correct slip boundary conditions for the compressible Navier-Stokes equations have been derived and used in some specific problems [2,3].

The paper is organized as follows. After this introduction, the problem is stated in Sect. 2 and formulated both in dimensional and dimensionless forms in Sect. 3. The Chapman-Enskog solution and the resulting compressible Navier-Stokes equations are summarized in Sect. 4. Section 5, which is the main part of the paper, is devoted to the derivation of the slip boundary conditions by means of the analysis of the Knudsen layer. The comparison of the obtained result with the existing formulas is also contained here (Sect. 5.3.4). The derived formulas of the slip boundary conditions, together with the compressible Navier-Stokes equations, are summarized in the dimensional form in Sect. 6, and concluding remarks are given in Sect. 7. 


\section{Problem and Assumptions}

Let us consider a monatomic ideal gas occupying a domain in contact with solid boundaries of arbitrary but smooth shape. The domain may extend to infinity, and no external force is acting on the gas. We investigate the unsteady behavior of the gas under the following assumptions:

(i) The behavior of the gas is described by the Boltzmann equation (the BGK model is also considered in parallel).

(ii) The boundaries do not deform and undergo a rigid-body motion, and the gas molecules are reflected on the boundaries according to the Maxwell type diffusespecular reflection.

(iii) The mean free path (or the mean free time) of the gas molecules at the reference equilibrium state at rest is sufficiently small compared to the characteristic length (or the characteristic time) of the system.

(iv) At the initial time, the boundary is at rest and has a uniform temperature, and the gas is in the equilibrium state at rest with the same temperature. After the initial time, the boundary can be set into motion smoothly, and the temperature of the boundary can change smoothly in time and position. (For the problems including infinities, the corresponding initial state and slow variations should be assumed at infinities.)

Assumption (iv) is to avoid the occurrence of the initial layer and that of the interaction between the initial layer and the Knudsen layer during the initial stage. It is for the purpose of theoretical rigor. However, we want to apply the resulting system of the compressible Navier-Stokes equations and the slip boundary conditions for more general initial conditions, admitting the inaccuracy during the initial stage with the duration of the order of the mean free time. This point will be commented on at the end of Sect. 5.2.4.

In the present manuscript, although the formulation is given with the Maxwell-type boundary condition [assumption (ii)], the coefficients in the slip boundary conditions will be given only for complete accommodation (diffuse reflection). We will consider the case of the Maxwell-type boundary condition in a separate paper, restricting ourselves to the BGK model and focusing our attention on the numerical method for the Knudsen-layer problems.

\section{Formulation of the Problem}

\subsection{Basic Equations}

Let us denote by $f(t, \boldsymbol{X}, \boldsymbol{\xi})$ the velocity distribution function of the gas molecules, where $t$ is the time variable, $\boldsymbol{X}$ (or $X_{i}$ ) is the position vector in the physical space, and $\boldsymbol{\xi}$ (or $\xi_{i}$ ) is the molecular velocity. Then, the mass density $\rho$, flow velocity $\boldsymbol{v}$ (or $v_{i}$ ), temperature $T$, pressure $p$, stress tensor $p_{i j}$, and heat-flow vector $\boldsymbol{q}$ (or $q_{i}$ ), which are all 
functions of $t$ and $\boldsymbol{X}$, are expressed as appropriate moments of the velocity distribution function, that is,

$$
\begin{aligned}
\rho & =\int f(t, \boldsymbol{X}, \boldsymbol{\xi}) d \boldsymbol{\xi}, \\
v_{i} & =\frac{1}{\rho} \int \xi_{i} f(t, \boldsymbol{X}, \boldsymbol{\xi}) d \boldsymbol{\xi}, \\
T & =\frac{1}{3 \rho R} \int|\boldsymbol{\xi}-\boldsymbol{v}|^{2} f(t, \boldsymbol{X}, \boldsymbol{\xi}) d \boldsymbol{\xi}, \\
p & =\frac{1}{3} \int|\boldsymbol{\xi}-\boldsymbol{v}|^{2} f(t, \boldsymbol{X}, \boldsymbol{\xi}) d \boldsymbol{\xi}=R \rho T, \\
p_{i j} & =\int\left(\xi_{i}-v_{i}\right)\left(\xi_{j}-v_{j}\right) f(t, \boldsymbol{X}, \boldsymbol{\xi}) d \boldsymbol{\xi}, \\
q_{i} & =\frac{1}{2} \int\left(\xi_{i}-v_{i}\right)|\boldsymbol{\xi}-\boldsymbol{v}|^{2} f(t, \boldsymbol{X}, \boldsymbol{\xi}) d \boldsymbol{\xi},
\end{aligned}
$$

where $R$ is the gas constant per unit mass $\left(R=k_{\mathrm{B}} / m\right.$ with the Boltzmann constant $k_{\mathrm{B}}$ and the mass $m$ of a molecule), $d \boldsymbol{\xi}=d \xi_{1} d \xi_{2} d \xi_{3}$, and the domain of integration with respect to $\boldsymbol{\xi}$ is its whole space.

The equation for $f$ is the Boltzmann equation [56, 57]:

$$
\frac{\partial f}{\partial t}+\xi_{i} \frac{\partial f}{\partial X_{i}}=J(f, f)
$$

where $J$ is defined with arbitrary functions $g(\boldsymbol{\xi})$ and $h(\boldsymbol{\xi})$ of $\boldsymbol{\xi}$ as follows:

$$
J(g, h)=\frac{1}{2 m} \int_{\boldsymbol{\alpha} \in \mathbb{S}^{2}, \boldsymbol{\xi}_{*} \in \mathbb{R}^{3}}\left(g^{\prime} h_{*}^{\prime}+g_{*}^{\prime} h^{\prime}-g h_{*}-g_{*} h\right) B d \Omega(\boldsymbol{\alpha}) d \boldsymbol{\xi}_{*} .
$$

Here, the usual convention, $g=g(\boldsymbol{\xi}), g_{*}=g\left(\boldsymbol{\xi}_{*}\right), g^{\prime}=g\left(\boldsymbol{\xi}^{\prime}\right)$, and $g_{*}^{\prime}=g\left(\boldsymbol{\xi}_{*}^{\prime}\right)$ and the same for $h$, is used; when a pair of molecules with velocities $\boldsymbol{\xi}$ and $\boldsymbol{\xi}_{*}$ collide, the velocities of the respective molecules after collision, $\boldsymbol{\xi}^{\prime}$ and $\boldsymbol{\xi}_{*}^{\prime}$, are expressed as

$$
\boldsymbol{\xi}^{\prime}=\boldsymbol{\xi}+\left[\left(\boldsymbol{\xi}_{*}-\boldsymbol{\xi}\right) \cdot \boldsymbol{\alpha}\right] \boldsymbol{\alpha}, \quad \boldsymbol{\xi}_{*}^{\prime}=\boldsymbol{\xi}_{*}-\left[\left(\boldsymbol{\xi}_{*}-\boldsymbol{\xi}\right) \cdot \boldsymbol{\alpha}\right] \boldsymbol{\alpha},
$$

where $\boldsymbol{\alpha}$, which is an integration variable together with $\boldsymbol{\xi}_{*}$, is the unit vector in the direction of $\boldsymbol{\xi}^{\prime}-\boldsymbol{\xi} ; d \boldsymbol{\xi}_{*}=d \boldsymbol{\xi}_{* 1} d \boldsymbol{\xi}_{* 2} d \boldsymbol{\xi}_{* 3}$ and $d \Omega(\boldsymbol{\alpha})$ is the solid-angle element around $\boldsymbol{\alpha} ; B$ is the non-negative function of $\left|\boldsymbol{\alpha} \cdot\left(\boldsymbol{\xi}_{*}-\boldsymbol{\xi}\right)\right| /\left|\boldsymbol{\xi}_{*}-\boldsymbol{\xi}\right|$ and $\left|\boldsymbol{\xi}_{*}-\boldsymbol{\xi}\right|$, i.e.,

$$
B=B\left(\frac{\left|\boldsymbol{\alpha} \cdot\left(\boldsymbol{\xi}_{*}-\boldsymbol{\xi}\right)\right|}{\left|\boldsymbol{\xi}_{*}-\boldsymbol{\xi}\right|},\left|\boldsymbol{\xi}_{*}-\boldsymbol{\xi}\right|\right),
$$

which depends on the intermolecular potential, and for hard-sphere molecules, $B=$ $d_{m}^{2}\left|\boldsymbol{\alpha} \cdot\left(\boldsymbol{\xi}_{*}-\boldsymbol{\xi}\right)\right| / 2$ with $d_{m}$ being the diameter of a molecule.

For the BGK model, the collision operator $J(f, f)$ in (2) is replaced by the following $J_{\mathrm{BGK}}(f)$ :

$$
J_{\mathrm{BGK}}(f)=A_{c}(T) \rho\left(f_{e}-f\right),
$$


where $f_{e}$ is a local Maxwellian

$$
f_{e}=\frac{\rho}{(2 \pi R T)^{3 / 2}} \exp \left(-\frac{|\boldsymbol{\xi}-\boldsymbol{v}|^{2}}{2 R T}\right),
$$

and $\rho, \boldsymbol{v}$, and $T$ are defined by (1a)-(1c). In (6), $A_{c}(T)$ is a function of the temperature $T$ such that $A_{c}(T) \rho$ is the collision frequency of a molecule with velocity $\xi$. Thus, the collision frequency is independent of $\boldsymbol{\xi}$ in this model. When $A_{c}(T)$ is a constant, the model corresponds to the pseudo-Maxwell molecule.

The initial condition [cf. assumption (iv)] is given at time $t=0$ by

$$
f(0, \boldsymbol{X}, \boldsymbol{\xi})=\frac{\rho_{0}}{\left(2 \pi R T_{0}\right)^{3 / 2}} \exp \left(-\frac{|\boldsymbol{\xi}|^{2}}{2 R T_{0}}\right),
$$

where $\rho_{0}$ is the reference density, and $T_{0}$ is the reference temperature.

The boundary condition [cf. assumption (ii)] is written as

$$
\begin{aligned}
& f\left(t, \boldsymbol{X}_{\mathrm{w}}, \boldsymbol{\xi}\right)=(1-\alpha) \operatorname{R} f\left(t, \boldsymbol{X}_{\mathrm{w}}, \boldsymbol{\xi}\right)+\alpha \frac{\rho_{\mathrm{w}}}{\left(2 \pi R T_{\mathrm{w}}\right)^{3 / 2}} \exp \left(-\frac{\left|\boldsymbol{\xi}-\boldsymbol{v}_{\mathrm{w}}\right|^{2}}{2 R T_{\mathrm{W}}}\right), \\
& \text { for }\left(\boldsymbol{\xi}-\boldsymbol{v}_{\mathrm{w}}\right) \cdot \boldsymbol{n}>0 \\
& \rho_{\mathrm{w}}=-\left(\frac{2 \pi}{R T_{\mathrm{w}}}\right)^{1 / 2} \int_{\left(\boldsymbol{\xi}-\boldsymbol{v}_{\mathrm{w}}\right) \cdot \boldsymbol{n}<0}\left(\boldsymbol{\xi}-\boldsymbol{v}_{\mathrm{w}}\right) \cdot \boldsymbol{n} f\left(t, \boldsymbol{X}_{\mathrm{w}}, \boldsymbol{\xi}\right) d \boldsymbol{\xi}
\end{aligned}
$$

where $\boldsymbol{X}_{\mathrm{w}}$ (or $X_{\mathrm{w} i}$ ) is the position of a point on the boundary, $\boldsymbol{v}_{\mathrm{w}}\left(\right.$ or $v_{\mathrm{w} i}$ ) and $T_{\mathrm{w}}$ are the velocity and temperature of the boundary at the point $\boldsymbol{X}_{\mathrm{w}}$, and $\boldsymbol{n}$ is the unit normal vector to the boundary, pointing into the gas, at $\boldsymbol{X}_{\mathrm{w}}$. In general, $\boldsymbol{X}_{\mathrm{w}}$ is a function of $t, \boldsymbol{v}_{\mathrm{w}}$ is the time derivative of $\boldsymbol{X}_{\mathrm{w}}$, and the arguments of $T_{\mathrm{w}}$ and $\boldsymbol{n}$ are $\left(t, \boldsymbol{X}_{\mathrm{w}}\right)$. In accordance with assumption (iv), $\boldsymbol{v}_{\mathrm{w}}=0$ and $T_{\mathrm{w}}=T_{0}$ at $t=0$, and $\boldsymbol{X}_{\mathrm{w}}$ (thus, $\boldsymbol{v}_{\mathrm{w}}$ ), $T_{\mathrm{w}}$, and $\boldsymbol{n}$ are assumed to change smoothly with $t$. In (9a), $\mathrm{R}$ is the reflection operator defined by

$$
\operatorname{Rg}\left(\xi_{i}\right)=g\left(\xi_{i}-2\left(\xi_{j}-v_{\mathrm{w} j}\right) n_{j} n_{i}\right),
$$

with an arbitrary function $g(\boldsymbol{\xi})$ of $\boldsymbol{\xi}$, and $\alpha(0 \leq \alpha \leq 1)$ is the so-called accommodation coefficient, giving the specular reflection when $\alpha=0$ and the diffuse reflection when $\alpha=1$. In the present paper, we assume that $\alpha=O(1)$, so that the case of specular or almost specular reflection is excluded. When $\alpha=0$ or $\alpha=O(\varepsilon)$, we need a Knudsen-layer analysis of different type, and the resulting slip boundary conditions are of different form from those for $\alpha=O(1)$ (see [58, 1, 4]). Note that the boundary condition (9) satisfies the condition that there is no instantaneous mass flow across the boundary, i.e.,

$$
\int\left(\boldsymbol{\xi}-\boldsymbol{v}_{\mathrm{w}}\right) \cdot \boldsymbol{n} f\left(t, \boldsymbol{X}_{\mathrm{w}}, \boldsymbol{\xi}\right) d \boldsymbol{\xi}=0
$$

\subsection{Dimensionless Form}

In this subsection we nondimensionalize our basic system shown in Sect. 3.1. Let $L$ be the reference length, $t_{0}$ the reference time, and $p_{0}=R \rho_{0} T_{0}$ the reference pressure, 
where $\rho_{0}$ and $T_{0}$ are the reference density and temperature appeared in (8). In the present study, we choose $t_{0}$ as

$$
t_{0}=L /\left(2 R T_{0}\right)^{1 / 2} .
$$

Then, we introduce the dimensionless quantities $\left(\hat{t}, x_{i}, \zeta_{i}, \hat{f}, \hat{\rho}, \hat{v}_{i}, \hat{T}, \hat{p}, \hat{p}_{i j}, \hat{q}_{i}, x_{\mathrm{w} i}\right.$, $\left.\hat{v}_{\mathrm{w} i}, \hat{T}_{\mathrm{w}}\right)$, corresponding to the dimensional quantities $\left(t, X_{i}, \xi_{i}, f, \rho, v_{i}, T, p, p_{i j}, q_{i}\right.$, $\left.X_{\mathrm{w} i}, v_{\mathrm{w} i}, T_{\mathrm{w}}\right)$, by the following relations:

$$
\begin{aligned}
& \hat{t}=t / t_{0}, \quad x_{i}=X_{i} / L, \quad \zeta_{i}=\xi_{i} /\left(2 R T_{0}\right)^{1 / 2}, \\
& \hat{f}=f /\left[\rho_{0} /\left(2 R T_{0}\right)^{3 / 2}\right], \quad \hat{\rho}=\rho / \rho_{0}, \quad \hat{v}_{i}=v_{i} /\left(2 R T_{0}\right)^{1 / 2}, \\
& \hat{T}=T / T_{0}, \quad \hat{p}=p / p_{0}, \quad \hat{p}_{i j}=p_{i j} / p_{0}, \quad \hat{q}_{i}=q_{i} / p_{0}\left(2 R T_{0}\right)^{1 / 2}, \\
& x_{\mathrm{w} i}=X_{\mathrm{w} i} / L, \quad \hat{v}_{\mathrm{w} i}=v_{\mathrm{w} i} /\left(2 R T_{0}\right)^{1 / 2}, \quad \hat{T}_{\mathrm{w}}=T_{\mathrm{w}} / T_{0} .
\end{aligned}
$$

We also use $\boldsymbol{x}, \boldsymbol{\zeta}, \hat{\boldsymbol{v}}, \hat{\boldsymbol{q}}, \boldsymbol{x}_{\mathrm{w}}$, and $\hat{\boldsymbol{v}}_{\mathrm{w}}$ for $x_{i}, \zeta_{i}, \hat{v}_{i}, \hat{q}_{i}, x_{\mathrm{w} i}$, and $\hat{v}_{\mathrm{w} i}$, respectively.

Then, corresponding to (1), we have the following relations between the macroscopic quantities and the velocity distribution function:

$$
\begin{aligned}
& \hat{\rho}=\int \hat{f} d \boldsymbol{\zeta}, \\
& \hat{v}_{i}=\frac{1}{\hat{\rho}} \int \zeta_{i} \hat{f} d \boldsymbol{\zeta}, \\
& \hat{T}=\frac{2}{3 \hat{\rho}} \int|\boldsymbol{\zeta}-\hat{\boldsymbol{v}}|^{2} \hat{f} d \boldsymbol{\zeta}, \\
& \hat{p}=\frac{2}{3} \int|\boldsymbol{\zeta}-\hat{\boldsymbol{v}}|^{2} \hat{f} d \boldsymbol{\zeta}=\hat{\rho} \hat{T}, \\
& \hat{p}_{i j}=2 \int\left(\zeta_{i}-\hat{v}_{i}\right)\left(\zeta_{j}-\hat{v}_{j}\right) \hat{f} d \boldsymbol{\zeta}, \\
& \hat{q}_{i}=\int\left(\zeta_{i}-\hat{v}_{i}\right)|\boldsymbol{\zeta}-\hat{\boldsymbol{v}}|^{2} \hat{f} d \boldsymbol{\zeta},
\end{aligned}
$$

where $d \zeta=d \zeta_{1} d \zeta_{2} d \zeta_{3}$, and the domain of integration with respect to $\zeta$ is its whole space. In the following, if the domain of integration is omitted, it means that the integration is over the whole space of the integration variables.

The dimensionless form of the Boltzmann equation $(2)$ reads $[56,57]$

$$
\frac{\partial \hat{f}}{\partial \hat{t}}+\zeta_{i} \frac{\partial \hat{f}}{\partial x_{i}}=\frac{1}{\varepsilon} \hat{J}(\hat{f}, \hat{f})
$$

where $\hat{J}$ is defined with arbitrary functions $\hat{g}(\boldsymbol{\zeta})$ and $\hat{h}(\boldsymbol{\zeta})$ of $\zeta$ by

$$
\hat{J}(\hat{g}, \hat{h})=\frac{1}{2} \int_{\boldsymbol{\alpha} \in \mathbb{S}^{2}, \zeta_{*} \in \mathbb{R}^{3}}\left(\hat{g}^{\prime} \hat{h}_{*}^{\prime}+\hat{g}_{*}^{\prime} \hat{h}^{\prime}-\hat{g} \hat{h}_{*}-\hat{g}_{*} \hat{h}\right) \hat{B} d \Omega(\boldsymbol{\alpha}) d \boldsymbol{\zeta}_{*} .
$$

Here, $d \boldsymbol{\zeta}_{*}=d \zeta_{* 1} d \zeta_{* 2} d \zeta_{* 3}$, and the same convention as in (3) is used, i.e., $\hat{g}=\hat{g}(\boldsymbol{\zeta})$, $\hat{g}_{*}=\hat{g}\left(\boldsymbol{\zeta}_{*}\right), \hat{g}^{\prime}=\hat{g}\left(\boldsymbol{\zeta}^{\prime}\right), \hat{g}_{*}^{\prime}=\hat{g}\left(\boldsymbol{\zeta}_{*}^{\prime}\right)$, etc.; the relation between $\left(\boldsymbol{\zeta}^{\prime}, \boldsymbol{\zeta}_{*}^{\prime}\right)$ and $\left(\boldsymbol{\zeta}, \boldsymbol{\zeta}_{*}\right)$, which corresponds to (4), is given as

$$
\zeta^{\prime}=\zeta+\left[\left(\boldsymbol{\zeta}_{*}-\zeta\right) \cdot \boldsymbol{\alpha}\right] \boldsymbol{\alpha}, \quad \boldsymbol{\zeta}_{*}^{\prime}=\boldsymbol{\zeta}_{*}-\left[\left(\boldsymbol{\zeta}_{*}-\boldsymbol{\zeta}\right) \cdot \boldsymbol{\alpha}\right] \boldsymbol{\alpha}
$$


the non-negative function $\hat{B}$, which is the dimensionless counterpart of $B$, is defined as

$$
\begin{aligned}
& \hat{B}=\hat{B}\left(\frac{\left|\boldsymbol{\alpha} \cdot\left(\boldsymbol{\zeta}_{*}-\boldsymbol{\zeta}\right)\right|}{\left|\boldsymbol{\zeta}_{*}-\boldsymbol{\zeta}\right|},\left|\boldsymbol{\zeta}_{*}-\boldsymbol{\zeta}\right|\right)=\frac{1}{B_{0}} B\left(\frac{\left|\boldsymbol{\alpha} \cdot\left(\boldsymbol{\xi}_{*}-\boldsymbol{\xi}\right)\right|}{\left|\boldsymbol{\xi}_{*}-\boldsymbol{\xi}\right|},\left|\boldsymbol{\xi}_{*}-\boldsymbol{\xi}\right|\right) \\
& B_{0}=\frac{1}{\rho_{0}^{2}} \int f_{0} f_{0 *} B\left(\frac{\left|\boldsymbol{\alpha} \cdot\left(\boldsymbol{\xi}_{*}-\boldsymbol{\xi}\right)\right|}{\left|\boldsymbol{\xi}_{*}-\boldsymbol{\xi}\right|},\left|\boldsymbol{\xi}_{*}-\boldsymbol{\xi}\right|\right) d \Omega(\boldsymbol{\alpha}) d \boldsymbol{\xi} d \boldsymbol{\xi}_{*}
\end{aligned}
$$

where $f_{0}(\boldsymbol{\xi})$ is the Maxwellian for the equilibrium state at rest at density $\rho_{0}$ and temperature $T_{0}$, i.e., $f_{0}(\boldsymbol{\xi})=\rho_{0}\left(2 \pi R T_{0}\right)^{-3 / 2} \exp \left(-|\boldsymbol{\xi}|^{2} / 2 R T_{0}\right)$, and $f_{0 *}=f_{0}\left(\boldsymbol{\xi}_{*}\right) ; \rho_{0} B_{0} / m$ indicates the mean collision frequency $\overline{v_{c}}$ of the gas molecules in the equilibrium state at rest with density $\rho_{0}$ and temperature $T_{0}$; the parameter $\varepsilon$ in (15) is defined by

$$
\varepsilon=(\sqrt{\pi} / 2) \mathrm{Kn}=(\sqrt{\pi} / 2)\left(l_{0} / L\right),
$$

where $\mathrm{Kn}=l_{0} / L$ is the Knudsen number, and $l_{0}$ is the mean free path of the gas molecules defined by

$$
l_{0}=(2 / \sqrt{\pi})\left(2 R T_{0}\right)^{1 / 2} / \overline{v_{c}}=(2 / \sqrt{\pi})\left(2 R T_{0}\right)^{1 / 2}\left(m / \rho_{0} B_{0}\right) .
$$

For hard-sphere molecules, $B_{0}, \hat{B}$, and $l_{0}$ become

$$
B_{0}=4 \sqrt{\pi} d_{m}^{2}\left(R T_{0}\right)^{1 / 2}, \quad \hat{B}=\left|\boldsymbol{\alpha} \cdot\left(\boldsymbol{\zeta}_{*}-\boldsymbol{\zeta}\right)\right| / 4(2 \pi)^{1 / 2}, \quad l_{0}=m / \sqrt{2} \pi d_{m}^{2} \rho_{0} .
$$

For an intermolecular force that extends to infinity, an appropriate cut off should be introduced in the integral in (18b) in order that $B_{0}$ takes a finite value.

For the BGK model, $\hat{J}(\hat{f}, \hat{f})$ in (15) is replaced by the following $\hat{J}_{\mathrm{BGK}}(\hat{f})$, which corresponds to (6):

$$
\hat{J}_{\mathrm{BGK}}(\hat{f})=\hat{A}_{c}(\hat{T}) \hat{\rho}\left(\hat{f}_{e}-\hat{f}\right)
$$

where $\hat{A}_{c}(\hat{T})=A_{c}(T) / A_{c}\left(T_{0}\right)$, so that $\hat{A}_{c}(1)=1$ and $\hat{A}_{c}=1$ when $A_{c}$ is a constant; $\hat{f}_{e}$ is a local Maxwellian

$$
\hat{f}_{e}=\frac{\hat{\rho}}{(\pi \hat{T})^{3 / 2}} \exp \left(-\frac{|\boldsymbol{\zeta}-\hat{\boldsymbol{v}}|^{2}}{\hat{T}}\right),
$$

and $\hat{\rho}, \hat{v}_{i}$, and $\hat{T}$ are defined by (14a)-(14c). In this model, since the collision frequency of a molecule with velocity $\boldsymbol{\xi}$ is $A_{c} \rho$ independent of $\boldsymbol{\xi}$ [cf. (6)], the mean collision frequency $\overline{v_{c}}$ in the equilibrium state at rest at density $\rho_{0}$ and temperature $T_{0}$ is $\overline{v_{c}}=$ $A_{c}\left(T_{0}\right) \rho_{0}$. Therefore, it follows from the first equality in (20) that

$$
l_{0}=(2 / \sqrt{\pi})\left(2 R T_{0}\right)^{1 / 2} / A_{c}\left(T_{0}\right) \rho_{0} .
$$

The dimensionless form of the initial condition (8) is given by

$$
\hat{f}(0, \boldsymbol{x}, \boldsymbol{\zeta})=E(\zeta)
$$


where

$$
\zeta=|\boldsymbol{\zeta}|=\left(\zeta_{i}^{2}\right)^{1 / 2}, \quad E(\zeta)=\pi^{-3 / 2} \exp \left(-\zeta^{2}\right),
$$

and the dimensionless form of the boundary condition (9) reads as follows:

$$
\begin{aligned}
& \hat{f}\left(\hat{t}, \boldsymbol{x}_{\mathrm{w}}, \boldsymbol{\zeta}\right)=(1-\alpha) \hat{\mathrm{R}} \hat{f}\left(\hat{t}, \boldsymbol{x}_{\mathrm{w}}, \boldsymbol{\zeta}\right)+\alpha \frac{\hat{\rho}_{\mathrm{w}}}{\left(\pi \hat{T}_{\mathrm{w}}\right)^{3 / 2}} \exp \left(-\frac{\left|\boldsymbol{\zeta}-\hat{\boldsymbol{v}}_{\mathrm{w}}\right|^{2}}{\hat{T}_{\mathrm{W}}}\right), \\
& \text { for }\left(\boldsymbol{\zeta}-\hat{\boldsymbol{v}}_{\mathrm{w}}\right) \cdot \boldsymbol{n}>0, \\
& \hat{\rho}_{\mathrm{w}}=-2\left(\frac{\pi}{\hat{T}_{\mathrm{w}}}\right)^{1 / 2} \int_{\left(\boldsymbol{\zeta}-\hat{\boldsymbol{v}}_{\mathrm{w}}\right) \cdot \boldsymbol{n}<0}\left(\boldsymbol{\zeta}-\hat{\boldsymbol{v}}_{\mathrm{W}}\right) \cdot \boldsymbol{n} \hat{f}\left(\hat{t}, \boldsymbol{x}_{\mathrm{w}}, \boldsymbol{\zeta}\right) d \boldsymbol{\zeta},
\end{aligned}
$$

where $\hat{R}$ is the dimensionless version of the reflection operator (10) that acts on any function $\hat{g}$ of $\zeta_{i}$, i.e.,

$$
\hat{\mathrm{R}} \hat{g}\left(\zeta_{i}\right)=\hat{g}\left(\zeta_{i}-2\left(\zeta_{j}-\hat{v}_{\mathrm{w} j}\right) n_{j} n_{i}\right) .
$$

We note that $\hat{\boldsymbol{v}}_{\mathrm{w}}=0$ and $\hat{T}_{\mathrm{w}}=1$ at $\hat{t}=0$, and $\boldsymbol{x}_{\mathrm{w}}$ (thus $\hat{\boldsymbol{v}}_{\mathrm{w}}$ ), $\hat{T}_{\mathrm{w}}$, and $\boldsymbol{n}$ are assumed to change smoothly in $\hat{t}$. Corresponding to (11), the following condition holds on the boundary:

$$
\int\left(\boldsymbol{\zeta}-\hat{\boldsymbol{v}}_{\mathrm{w}}\right) \cdot \boldsymbol{n} \hat{f}\left(\hat{t}, \boldsymbol{x}_{\mathrm{w}}, \boldsymbol{\zeta}\right) d \boldsymbol{\zeta}=0
$$

We analyze the Boltzmann equation (15) with conditions (25) and (27) when the Knudsen number $\mathrm{Kn}$ is small, that is, when $\varepsilon \ll 1$. Since our reference time is $t_{0}=$ $L /\left(2 R T_{0}\right)^{1 / 2}=1 / \varepsilon \overline{v_{c}}$, it is much larger than the mean free time $1 / \overline{v_{c}}$.

\section{Chapman-Enskog Solution and the Compressible Navier- Stokes Equations}

The Chapman-Enskog expansion is a well-known technique to derive the Euler and Navier-Stokes equations for a compressible fluid from the Boltzmann equation [16, 23, 14, 57]. In this section, we summarize the Chapman-Enskog solution and the resulting Navier-Stokes equations. The solution is based on the assumption that its length scale of variation is of $O(1)$ [or $O(L)$ in the dimensional space] and the initial and boundary conditions are not taken into account. Therefore, it should be distinguished from the correct solution $f$ for the initial and boundary value problem of the Boltzmann equation by an appropriate subscript, such as $f_{\mathrm{CE}}$. However, in order to avoid complexity of notation, we denote it just by $f$ in this and following sections. We basically follow the notation of Sect. B.4 in [57]. 


\subsection{Linearized Collision Operator}

This subsection is a preparation for the Chapman-Enskog solution. We first introduce the linearized collision operator $\mathscr{L}(\cdot)$ :

$$
\begin{aligned}
\mathscr{L}(\varphi) & =2 \hat{J}(E, E \varphi) / E \\
& =\int E\left(\zeta_{*}\right)\left(\varphi_{*}^{\prime}+\varphi^{\prime}-\varphi_{*}-\varphi\right) \hat{B}\left(\frac{\left|\left(\boldsymbol{\zeta}_{*}-\boldsymbol{\zeta}\right) \cdot \boldsymbol{\alpha}\right|}{\left|\boldsymbol{\zeta}_{*}-\boldsymbol{\zeta}\right|},\left|\boldsymbol{\zeta}_{*}-\boldsymbol{\zeta}\right|\right) d \Omega(\boldsymbol{\alpha}) d \boldsymbol{\zeta}_{*}
\end{aligned}
$$

where $\zeta_{*}=\left|\zeta_{*}\right|=\left(\zeta_{* i}^{2}\right)^{1 / 2}, E$ indicates the function defined by $(26), \varphi(\zeta)$ is an arbitrary function of $\zeta$, and the same convention as in (16) is used, i.e., $\varphi_{*}=\varphi\left(\zeta_{*}\right)$, $\varphi^{\prime}=\varphi\left(\zeta^{\prime}\right)$, etc. Then, we define the following extended linearized collision operator $\mathscr{L}_{a}(\cdot)$ :

$$
\mathscr{L}_{a}(\varphi)=\int E\left(\zeta_{*}\right)\left(\varphi_{*}^{\prime}+\varphi^{\prime}-\varphi_{*}-\varphi\right) \hat{B}_{a}\left(\frac{\left|\left(\boldsymbol{\zeta}_{*}-\boldsymbol{\zeta}\right) \cdot \boldsymbol{\alpha}\right|}{\left|\boldsymbol{\zeta}_{*}-\boldsymbol{\zeta}\right|},\left|\boldsymbol{\zeta}_{*}-\boldsymbol{\zeta}\right|\right) d \Omega(\boldsymbol{\alpha}) d \boldsymbol{\zeta}_{*}
$$

where

$$
\hat{B}_{a}=\hat{B}_{a}\left(\frac{\left|\boldsymbol{\alpha} \cdot\left(\boldsymbol{\zeta}_{*}-\boldsymbol{\zeta}\right)\right|}{\left|\boldsymbol{\zeta}_{*}-\boldsymbol{\zeta}\right|},\left|\boldsymbol{\zeta}_{*}-\boldsymbol{\zeta}\right|\right)=\frac{1}{\sqrt{a}} \hat{B}\left(\frac{\left|\boldsymbol{\alpha} \cdot\left(\boldsymbol{\zeta}_{*}-\boldsymbol{\zeta}\right)\right|}{\left|\boldsymbol{\zeta}_{*}-\boldsymbol{\zeta}\right|}, \sqrt{a}\left|\boldsymbol{\zeta}_{*}-\boldsymbol{\zeta}\right|\right),
$$

and $a$ is a positive quantity independent of $\zeta$. Obviously, $\mathscr{L}_{1}(\varphi)=\mathscr{L}(\varphi)$ holds, and for hard-sphere molecules, $\mathscr{L}_{a}(\varphi)=\mathscr{L}(\varphi)$ for any $a$.

The linearized collision operators $\mathscr{L}_{a \mathrm{BGK}}(\cdot)$ and $\mathscr{L}_{\mathrm{BGK}}(\cdot)$ for the BGK model, which correspond to $\mathscr{L}_{a}(\cdot)$ and $\mathscr{L}(\cdot)$, respectively, take the following forms:

$$
\begin{aligned}
& \mathscr{L}_{a \mathrm{BGK}}(\varphi)=\frac{\hat{A}_{c}(a)}{\sqrt{a}} \mathscr{L}_{\mathrm{BGK}}(\varphi), \\
& \mathscr{L}_{\mathrm{BGK}}(\varphi)=\int\left[1+2 \zeta \cdot \zeta_{*}+\frac{2}{3}\left(\zeta^{2}-\frac{3}{2}\right)\left(\zeta_{*}^{2}-\frac{3}{2}\right)\right] \varphi\left(\boldsymbol{\zeta}_{*}\right) E\left(\zeta_{*}\right) d \zeta_{*}-\varphi(\zeta),
\end{aligned}
$$

where $\hat{A}_{c}(1)=1$ [cf. (22)], so that $\mathscr{L}_{1 \mathrm{BGK}}(\varphi)=\mathscr{L}_{\mathrm{BGK}}(\varphi)$.

In the Chapman-Enskog solution in Sect. 4.2, the following two functions $\mathscr{A}(\zeta, a)$ and $\mathscr{B}^{(0)}(\zeta, a)$ appear: $\mathscr{A}(\zeta, a)$ is the solution of the integral equation

$$
\mathscr{L}_{a}\left[\zeta_{i} \mathscr{A}(\zeta, a)\right]=-\zeta_{i}\left(\zeta^{2}-\frac{5}{2}\right)
$$

with the subsidiary condition

$$
\int_{0}^{\infty} \zeta^{4} \mathscr{A}(\zeta, a) E(\zeta) d \zeta=0
$$

and the function $\mathscr{B}^{(0)}(\zeta, a)$ is the solution of the integral equation

$$
\mathscr{L}_{a}\left[\left(\zeta_{i} \zeta_{j}-\frac{1}{3} \zeta^{2} \delta_{i j}\right) \mathscr{B}^{(0)}(\zeta, a)\right]=-2\left(\zeta_{i} \zeta_{j}-\frac{1}{3} \zeta^{2} \delta_{i j}\right),
$$


where $\delta_{i j}$ is the Kronecker delta.

In [57], $\mathscr{A}(\zeta, 1)$ is denoted by $A(\zeta)$, and $\mathscr{B}^{(0)}(\zeta, 1)$ by $B(\zeta)$ :

$$
\mathscr{A}(\zeta, 1)=A(\zeta), \quad \mathscr{B}^{(0)}(\zeta, 1)=B(\zeta) .
$$

Thus, for hard-sphere molecules, $\mathscr{A}(\zeta, a)=A(\zeta)$ and $\mathscr{B}^{(0)}(\zeta, a)=B(\zeta)$ for any $a$. The numerical values of $A(\zeta)$ and $B(\zeta)$ for hard-sphere molecules are tabulated in Table 3.1 of [57] (see also [40, 35]). For the BGK model, (34) [with (35)] and (36), with $\mathscr{L}_{a}=\mathscr{L}_{a \mathrm{BGK}}$, give the following solutions:

$$
\mathscr{A}(\zeta, a)=\frac{\sqrt{a}}{\hat{A}_{c}(a)}\left(\zeta^{2}-\frac{5}{2}\right), \quad \mathscr{B}^{(0)}(\zeta, a)=2 \frac{\sqrt{a}}{\hat{A}_{c}(a)} .
$$

\subsection{Chapman-Enskog Solution and Navier-Stokes Equations}

The first-order Chapman-Enskog solution can be expressed as

$$
\hat{f}=\hat{f}^{(0)}+\hat{f}^{(1)} \varepsilon+O\left(\varepsilon^{2}\right) .
$$

Here, the leading-order term $\hat{f}^{(0)}$ is a local Maxwellian distribution

$$
\hat{f}^{(0)}=\frac{\hat{\rho}}{(\pi \hat{T})^{3 / 2}} \exp \left(-\frac{|\boldsymbol{\zeta}-\hat{\boldsymbol{v}}|^{2}}{\hat{T}}\right)=\frac{\hat{\rho}}{\hat{T}^{3 / 2}} E(\mathscr{C}),
$$

and the first-order term $\hat{f}^{(1)}$ takes the following form:

$$
\begin{aligned}
\hat{f}^{(1)} & =\hat{f}^{(0)} \Psi \\
\Psi= & -\frac{1}{\hat{\rho} \hat{T}} \mathscr{C}_{j} \mathscr{A}(\mathscr{C}, \hat{T}) \frac{\partial \hat{T}}{\partial x_{j}} \\
& -\frac{1}{2 \hat{\rho} \hat{T}^{1 / 2}}\left(\mathscr{C}_{j} \mathscr{C}_{k}-\frac{1}{3} \mathscr{C}^{2} \delta_{j k}\right) \mathscr{B}^{(0)}(\mathscr{C}, \hat{T})\left(\frac{\partial \hat{v}_{k}}{\partial x_{j}}+\frac{\partial \hat{v}_{j}}{\partial x_{k}}\right),
\end{aligned}
$$

where

$$
\mathscr{C}_{i}=\frac{\zeta_{i}-\hat{v}_{i}}{\hat{T}^{1 / 2}}, \quad \mathscr{C}=|\mathscr{C}|=\left(\mathscr{C}_{j}^{2}\right)^{1 / 2}
$$

and $\mathscr{A}(\mathscr{C}, \hat{T})$ and $\mathscr{B}^{(0)}(\mathscr{C}, \hat{T})$ are the functions appeared in Sect. 4.1.

The expansion (39) is designed in such a way that the first-order term $\hat{f}^{(1)}$, as well as the higher-order terms, satisfies the constraint:

$$
\int\left(1, \zeta_{i}, \zeta^{2}\right)\left[\varepsilon \hat{f}^{(1)}+O\left(\varepsilon^{2}\right)\right] d \boldsymbol{\zeta}=0,
$$

so that $\hat{\rho}, \hat{v}_{i}$, and $\hat{T}$ contained in $\hat{f}^{(0)}$ are nothing but the density, the flow velocity, and the temperature associated with $\hat{f}$ of the expansion (39) [cf. (14)]. 
With (39), the dimensionless stress tensor $\hat{p}_{i j}$ and heat-flow vector $\hat{q}_{i}$ become

$$
\begin{aligned}
& \hat{p}_{i j}=\hat{p} \delta_{i j}-\varepsilon \Gamma_{1}(\hat{T})\left(\frac{\partial \hat{v}_{i}}{\partial x_{j}}+\frac{\partial \hat{v}_{j}}{\partial x_{i}}-\frac{2}{3} \frac{\partial \hat{v}_{k}}{\partial x_{k}} \delta_{i j}\right)+O\left(\varepsilon^{2}\right), \\
& \hat{q}_{i}=-\frac{5}{4} \varepsilon \Gamma_{2}(\hat{T}) \frac{\partial \hat{T}}{\partial x_{i}}+O\left(\varepsilon^{2}\right),
\end{aligned}
$$

where $\Gamma_{1}(\hat{T})$ and $\Gamma_{2}(\hat{T})$ are the functions of $\hat{T}$ given by

$$
\begin{aligned}
& \Gamma_{1}(\hat{T})=\frac{8}{15 \sqrt{\pi}} \hat{T}^{1 / 2} \int_{0}^{\infty} \mathscr{C}^{6} \mathscr{B}^{(0)}(\mathscr{C}, \hat{T}) e^{-\mathscr{C}^{2}} d \mathscr{C}, \\
& \Gamma_{2}(\hat{T})=\frac{16}{15 \sqrt{\pi}} \hat{T}^{1 / 2} \int_{0}^{\infty} \mathscr{C}^{6} \mathscr{A}(\mathscr{C}, \hat{T}) e^{-\mathscr{C}^{2}} d \mathscr{C},
\end{aligned}
$$

and are related to the viscosity $\mu$ and the thermal conductivity $\lambda$ as

$$
\begin{aligned}
& \mu=\frac{p_{0} L}{\left(2 R T_{0}\right)^{1 / 2}} \varepsilon \Gamma_{1}(\hat{T})=\frac{\sqrt{\pi}}{2} \frac{p_{0} l_{0}}{\left(2 R T_{0}\right)^{1 / 2}} \Gamma_{1}\left(\frac{T}{T_{0}}\right), \\
& \lambda=\frac{5}{4} \frac{p_{0}\left(2 R T_{0}\right)^{1 / 2} L}{T_{0}} \varepsilon \Gamma_{2}(\hat{T})=\frac{5 \sqrt{\pi}}{8} \frac{p_{0}\left(2 R T_{0}\right)^{1 / 2} l_{0}}{T_{0}} \Gamma_{2}\left(\frac{T}{T_{0}}\right) .
\end{aligned}
$$

For hard-sphere molecules, the data of the functions $\mathscr{A}(\zeta, a)=A(\zeta)$ and $\mathscr{B}^{(0)}(\zeta, a)$ $=B(\zeta)$ in $[57,35]$ enable us to compute the integrals in (45) numerically [57, 35]. As for the BGK model, we can compute the integrals analytically with the explicit form of $\mathscr{A}(\zeta, a)$ and $\mathscr{B}^{(0)}(\zeta, a)$ given by (38). As the result, we have the following expressions of $\Gamma_{1}(\hat{T})$ and $\Gamma_{2}(\hat{T})$ :

$$
\begin{aligned}
& \Gamma_{1}(\hat{T})=1.270042427 \hat{T}^{1 / 2}, \quad \Gamma_{2}(\hat{T})=1.922284066 \hat{T}^{1 / 2} \quad \text { (hard sphere) } \\
& \Gamma_{1}(\hat{T})=\Gamma_{2}(\hat{T})=\hat{T} / \hat{A}_{c}(\hat{T}) \quad(\mathrm{BGK}) .
\end{aligned}
$$

Using (21) and (47a) in (46), we have, for hard-sphere molecules,

$$
\mu=0.17913618 \times \frac{m R^{1 / 2}}{d_{m}^{2}} T^{1 / 2}, \quad \lambda=0.67783290 \times \frac{m R^{3 / 2}}{d_{m}^{2}} T^{1 / 2},
$$

which are proportional to $T^{1 / 2}$. It follows from (24), (46), and (47b) that, for the BGK model,

$$
\mu=\frac{R}{A_{c}(T)} T, \quad \lambda=\frac{5}{2} \frac{R^{2}}{A_{c}(T)} T,
$$

where $A_{c}(T)=A_{c}\left(T_{0}\right) \hat{A}_{c}(\hat{T})$ is used. When $A_{c}(T)$ does not depend on $T, \mu$ and $\lambda$ are proportional to $T$. Although (46) gives an impression that $\mu$ and $\lambda$ depend on the choice of the reference quantities $\rho_{0}$ and $T_{0}$, they do not, as seen from (48) and (49).

If we use (44) in the Maxwell transport equations, which are derived by integrating (15) times $\left(1, \zeta_{i}, \zeta^{2}\right)$ over the whole space of $\zeta_{i}$, and neglect the terms of $O\left(\varepsilon^{2}\right)$, we obtain the Navier-Stokes equations for compressible fluids, i.e.,

$$
\frac{\partial \hat{\rho}}{\partial \hat{t}}+\frac{\partial \hat{\rho} \hat{v}_{j}}{\partial x_{j}}=0
$$




$$
\begin{gathered}
\frac{\partial \hat{\rho} \hat{v}_{i}}{\partial \hat{t}}+\frac{\partial \hat{\rho} \hat{v}_{i} \hat{v}_{j}}{\partial x_{j}}=-\frac{1}{2} \frac{\partial \hat{p}}{\partial x_{i}}+\frac{\varepsilon}{2} \frac{\partial}{\partial x_{j}}\left[\Gamma_{1}(\hat{T})\left(\frac{\partial \hat{v}_{i}}{\partial x_{j}}+\frac{\partial \hat{v}_{j}}{\partial x_{i}}-\frac{2}{3} \frac{\partial \hat{v}_{k}}{\partial x_{k}} \delta_{i j}\right)\right] \\
\frac{\partial}{\partial \hat{t}}\left[\hat{\rho}\left(\frac{3}{2} \hat{T}+\hat{v}_{j}^{2}\right)\right]+\frac{\partial}{\partial x_{j}}\left[\hat{\rho} \hat{v}_{j}\left(\frac{5}{2} \hat{T}+\hat{v}_{k}^{2}\right)\right] \\
=\frac{5}{4} \varepsilon \frac{\partial}{\partial x_{j}}\left[\Gamma_{2}(\hat{T}) \frac{\partial \hat{T}}{\partial x_{j}}\right]+\varepsilon \frac{\partial}{\partial x_{j}}\left[\Gamma_{1}(\hat{T}) \hat{v}_{i}\left(\frac{\partial \hat{v}_{i}}{\partial x_{j}}+\frac{\partial \hat{v}_{j}}{\partial x_{i}}-\frac{2}{3} \frac{\partial \hat{v}_{k}}{\partial x_{k}} \delta_{i j}\right)\right],
\end{gathered}
$$

where $\hat{p}=\hat{\rho} \hat{T}[(14 \mathrm{~d})]$.

\section{Derivation of the Slip Boundary Conditions}

\subsection{Introduction of the Knudsen Layer}

In the first-order Chapman-Enskog solution (39), which corresponds to the NavierStokes equations (50), the initial and boundary conditions (25) and (27) are not taken into account. To be consistent with the fact that the term up to $O(\varepsilon)$ is considered in (39), we need to satisfy the initial and boundary conditions up to the order of $\varepsilon$.

Concerning the initial condition (25), if we assume

$$
\hat{\rho}=1, \quad \hat{\boldsymbol{v}}=0, \quad \hat{T}=1, \quad \text { at } \hat{t}=0,
$$

then the Chapman-Enskog solution (39) satisfies (25) up to $O(\varepsilon)$, since $\partial \hat{v}_{i} / \partial x_{j}=0$ and $\partial \hat{T} / \partial x_{i}=0$. Therefore, under assumption (iv) in Sect. 2, (51) gives the correct initial condition for (50).

Next, we try to satisfy the boundary conditions with the Chapman-Enskog solution (39). If we recall that the leading-order term $\hat{f}^{(0)}$ is a local Maxwellian [(40)], it can be made to satisfy (27) by assuming that

$$
\hat{\boldsymbol{v}}=\hat{\boldsymbol{v}}_{\mathrm{w}}, \quad \hat{T}=\hat{T}_{\mathrm{w}}, \quad \text { at } \boldsymbol{x}=\boldsymbol{x}_{\mathrm{w}} .
$$

On the other hand, in order that the first-order term $\hat{f}^{(1)}$ satisfies (27) at the order of $\varepsilon$, we need to impose the additional constraints:

$$
\frac{\partial \hat{v}_{i}}{\partial x_{j}}+\frac{\partial \hat{v}_{j}}{\partial x_{i}}=0, \quad \frac{\partial \hat{T}}{\partial x_{i}}=0, \quad \text { at } \boldsymbol{x}=\boldsymbol{x}_{\mathrm{w}} .
$$

However, the constraints on the boundary, (52) and (53), are too many as the boundary conditions for the Navier-Stokes equations (50). Therefore, this approach does not work. But, the fact that the choice (52) works at the zeroth order in $\varepsilon$ suggests that

$$
\hat{\boldsymbol{v}}-\hat{\boldsymbol{v}}_{\mathrm{w}}=O(\varepsilon), \quad \hat{T}-\hat{T}_{\mathrm{w}}=O(\varepsilon), \quad \text { at } \boldsymbol{x}=\boldsymbol{x}_{\mathrm{w}} .
$$

In order to obtain the solution satisfying the boundary condition, we need to introduce the kinetic boundary layer, the so-called Knudsen layer, with thickness of the order of $\varepsilon$ adjacent to the boundary [57]. Let us denote the Chapman-Enskog solution 
(39) by $\hat{f}_{\mathrm{CE}}$, the correction term inside the Knudsen layer by $\hat{f}_{\mathrm{K}}$, and the total solution that satisfies the boundary condition by $\hat{f}_{\text {tot }}$. Then, we write

$$
\hat{f}_{\mathrm{tot}}=\hat{f}_{\mathrm{CE}}+\hat{f}_{\mathrm{K}}
$$

Correspondingly, we let the macroscopic quantities be

$$
\hat{h}_{\mathrm{tot}}=\hat{h}_{\mathrm{CE}}+\hat{h}_{\mathrm{K}}
$$

where $\hat{h}$ indicates any of the dimensionless macroscopic quantities, i.e., $\hat{\rho}, \hat{\boldsymbol{v}}, \hat{T}$, etc., appeared in Sect. 3.2, and $\hat{h}_{\mathrm{CE}}$ indicates those macroscopic quantities associated with the Chapman-Enskog solution. Note that $\hat{\rho}, \hat{v}, \hat{T}$, etc. appeared in Sect. 4 belong to $\hat{h}_{\mathrm{CE}}$ although the subscript "CE" was not used there.

We assume the following properties for the correction term $\hat{f}_{\mathrm{K}}$ :

(a) $\hat{f}_{\mathrm{K}}$ is appreciable only in the Knudsen layer and vanishes rapidly away from the boundary.

(b) $\hat{f}_{\mathrm{K}}$ has a length scale of variation of the order of $\varepsilon$ (i.e., of the order of the mean free path $l_{0}$ in the dimensional physical space) in the direction normal to the boundary, that is, $\varepsilon n_{j} \partial \hat{f}_{\mathrm{K}} / \partial x_{j}=O\left(\hat{f}_{\mathrm{K}}\right)$.

(c) $\hat{f}_{\mathrm{K}}$ has a length scale of variation of the order of 1 (i.e., of the order of the reference length $L$ in the dimensional physical space) in the direction along the boundary.

(d) $\hat{f}_{\mathrm{K}}$ has a time scale of variation of the order of 1 (i.e., of the order of $t_{0}=$ $L /\left(2 R T_{0}\right)^{1 / 2}$ in the dimensional time), i.e., $\partial \hat{f}_{\mathrm{K}} / \partial \hat{t}=O\left(\hat{f}_{\mathrm{K}}\right)$.

These assumptions are validated from the obtained results.

If we substitute (55) and (56) into (14) (with $\hat{f}=\hat{f}_{\text {tot }}$ and $\hat{h}=\hat{h}_{\text {tot }}$ ) and use the fact that $\hat{f}_{\mathrm{CE}}$ and $\hat{h}_{\mathrm{CE}}$ also satisfy the same (14) (cf. Sect. 4.2), we obtain the expressions of the corrections $\hat{h}_{\mathrm{K}}$ inside the Knudsen layer in terms of $\hat{f}_{\mathrm{K}}$ and $\hat{h}_{\mathrm{CE}}$. Further, if we substitute (55) into (15) and take into account the fact that $\hat{f}_{\mathrm{CE}}$ is the solution of the same (15), we obtain the equation for the Knudsen-layer correction $\hat{f}_{\mathrm{K}}$. We also obtain the initial and boundary conditions for $\hat{f}_{\mathrm{K}}$ by inserting (55) in (25) and (27). We will perform these procedures in a concrete way taking into account the properties of $\hat{f}_{\mathrm{CE}}$ and $\hat{f}_{\mathrm{K}}$ in the following subsections.

\subsection{Knudsen-Layer Problem}

\subsubsection{Preliminaries}

Since the boundary condition (27) can be satisfied by the Chapman-Enskog solution (39) at the zeroth order by the choice (52), we assume that $\hat{f}_{\mathrm{K}}$ starts at the order of $\varepsilon$ and let

$$
\hat{f}_{\mathrm{K}}=\hat{f}_{\mathrm{K}}^{(1)} \varepsilon+R_{f} \varepsilon^{2}
$$


where $R_{f} \varepsilon^{2}$ indicates the remainder, and $R_{f}$ is of $O(1)$ and has the properties (a)-(d). Correspondingly, we put

$$
\hat{h}_{\mathrm{K}}=\hat{h}_{\mathrm{K}}^{(1)} \varepsilon+R_{h} \varepsilon^{2},
$$

where $R_{h} \varepsilon^{2}$ is the remainder corresponding to $R_{f} \varepsilon^{2}$. In accordance with (54), we also put

$$
\hat{\boldsymbol{v}}-\hat{\boldsymbol{v}}_{\mathrm{w}}=\check{\boldsymbol{v}} \varepsilon, \quad \hat{T}-\hat{T}_{\mathrm{w}}=\check{T} \varepsilon, \quad \text { at } \boldsymbol{x}=\boldsymbol{x}_{\mathrm{w}},
$$

where $\check{\boldsymbol{v}}$ and $\check{T}$ are the quantities of $O(1)$.

Substituting (55) and (56) with (57) and (58) into (14) and picking up the terms of $O(\varepsilon)$, we have the following expressions of $\hat{h}_{\mathrm{K}}^{(1)}$ :

$$
\begin{aligned}
\hat{\rho}_{\mathrm{K}}^{(1)} & =\int \hat{f}_{\mathrm{K}}^{(1)} d \boldsymbol{\zeta}, \\
\hat{v}_{\mathrm{K} i}^{(1)} & =\frac{1}{\hat{\rho}} \int\left(\zeta_{i}-\hat{v}_{i}\right) \hat{f}_{\mathrm{K}}^{(1)} d \boldsymbol{\zeta}, \\
\hat{T}_{\mathrm{K}}^{(1)} & =\frac{2}{3 \hat{\rho}} \int\left(|\boldsymbol{\zeta}-\hat{v}|^{2}-\frac{3}{2} \hat{T}\right) \hat{f}_{\mathrm{K}}^{(1)} d \boldsymbol{\zeta}, \\
\hat{p}_{\mathrm{K}}^{(1)} & =\hat{\rho} \hat{T}_{\mathrm{K}}^{(1)}+\hat{\rho}_{\mathrm{K}}^{(1)} \hat{T}^{(1)} \\
\hat{p}_{\mathrm{K} i j}^{(1)} & =2 \int\left(\zeta_{i}-\hat{v}_{i}\right)\left(\zeta_{j}-\hat{v}_{j}\right) \hat{f}_{\mathrm{K}}^{(1)} d \boldsymbol{\zeta}, \\
\hat{q}_{\mathrm{K} i}^{(1)} & =\int\left(\zeta_{i}-\hat{v}_{i}\right)\left(|\boldsymbol{\zeta}-\hat{\boldsymbol{v}}|^{2}-\frac{5}{2} \hat{T}\right) \hat{f}_{\mathrm{K}}^{(1)} d \boldsymbol{\zeta} .
\end{aligned}
$$

Note again that $\hat{\rho}, \hat{\boldsymbol{v}}$, and $\hat{T}$ here are the macroscopic quantities associated with the Chapman-Enskog solution.

If we substitute (55) with (57) into (15) and note that $\hat{f}_{\mathrm{CE}}$ is the solution of (15), we obtain the following equation for $\hat{f}_{\mathrm{K}}^{(1)}$ :

$$
\varepsilon \frac{\partial \hat{f}_{\mathrm{K}}^{(1)}}{\partial \hat{t}}+\varepsilon \zeta_{i} \frac{\partial \hat{f}_{\mathrm{K}}^{(1)}}{\partial x_{i}}=2 \hat{J}\left(\hat{f}^{(0)}, \hat{f}_{\mathrm{K}}^{(1)}\right)+O\left(\varepsilon R_{f}\right)
$$

We will elaborate on this equation below.

\subsubsection{Knudsen-Layer Equation}

We first express a point $\boldsymbol{x}_{\mathrm{w}}$ on the boundary as a function of coordinates $\chi_{1}$ and $\chi_{2}$ fixed on the surface of the boundary and of time $\hat{t}$, i.e.,

$$
\boldsymbol{x}_{\mathrm{w}}=\boldsymbol{x}_{\mathrm{w}}\left(\hat{t}, \chi_{1}, \chi_{2}\right)
$$

[See Fig. 1(a).] When $\chi_{1}$ and $\chi_{2}$ are fixed, the function $\boldsymbol{x}_{\mathrm{w}}\left(\hat{t}, \chi_{1}, \chi_{2}\right)$ of $\hat{t}$ gives the trajectory of a fixed point on the boundary, and when $\hat{t}$ is fixed, the function $\boldsymbol{x}_{\mathrm{w}}\left(\hat{t}, \chi_{1}, \chi_{2}\right)$ of $\chi_{1}$ and $\chi_{2}$ gives the parameter representation of the boundary surface at time $\hat{t}$. The 
Figure 1: Coordinate systems. (a) Coordinate system on the boundary, (b) coordinate system for the Knudsen layer.

velocity of the boundary $\hat{\boldsymbol{v}}_{\mathrm{w}}$ and the unit normal vector to the boundary $\boldsymbol{n}$, which are also the functions of $\hat{t}, \chi_{1}$, and $\chi_{2}$, are expressed as

$$
\begin{aligned}
& \hat{\boldsymbol{v}}_{\mathrm{w}}\left(\hat{t}, \chi_{1}, \chi_{2}\right)=\frac{\partial \boldsymbol{x}_{\mathrm{w}}}{\partial \hat{t}}, \\
& \boldsymbol{n}\left(\hat{t}, \chi_{1}, \chi_{2}\right)= \pm\left(\frac{\partial \boldsymbol{x}_{\mathrm{w}}}{\partial \chi_{1}} \times \frac{\partial \boldsymbol{x}_{\mathrm{w}}}{\partial \chi_{2}}\right)\left|\frac{\partial \boldsymbol{x}_{\mathrm{w}}}{\partial \chi_{1}} \times \frac{\partial \boldsymbol{x}_{\mathrm{w}}}{\partial \chi_{2}}\right|^{-1},
\end{aligned}
$$

where $x$ indicates a vector product, and + sign or - sign is chosen in such a way that $\boldsymbol{n}$ points into gas region.

In order to analyze the Knudsen layer, we need to introduce a new coordinate system that is local near the boundary and appropriate to describe the rapid change of the physical quantities in the direction normal to the boundary. Let us introduce the new variables $\tilde{t}, \eta$, and $\zeta_{\mathrm{w}}$ by the following relations [see Fig. 1(b)]:

$$
\begin{aligned}
& \hat{t}=\tilde{t}, \\
& \boldsymbol{x}=\varepsilon \eta \boldsymbol{n}\left(\tilde{t}, \chi_{1}, \chi_{2}\right)+\boldsymbol{x}_{\mathrm{w}}\left(\tilde{t}, \chi_{1}, \chi_{2}\right), \\
& \boldsymbol{\zeta}=\boldsymbol{\zeta}_{\mathrm{w}}+\hat{\boldsymbol{v}}_{\mathrm{w}}\left(\tilde{t}, \chi_{1}, \chi_{2}\right) .
\end{aligned}
$$

Here, $\eta$ is a stretched normal coordinate, and $\zeta_{\mathrm{w}}$ is the molecular velocity relative to the velocity of the boundary. In accordance with the properties (a)-(d) in Sect. 5.1, we assume that $\hat{f}_{\mathrm{K}}$ is a function of $\left(\tilde{t}, \eta, \chi_{1}, \chi_{2}, \zeta_{\mathrm{w}}\right)$ and vanishes rapidly as $\eta \rightarrow \infty$ :

$$
\begin{aligned}
& \hat{f}_{\mathrm{K}}=\hat{f}_{\mathrm{K}}\left(\tilde{t}, \eta, \chi_{1}, \chi_{2}, \zeta_{\mathrm{w}}\right), \\
& \hat{f}_{\mathrm{K}} \rightarrow 0, \quad \text { as } \eta \rightarrow \infty .
\end{aligned}
$$

Therefore, (65) also holds for $\hat{f}_{\mathrm{K}}^{(1)}$ and $R_{f}$ in (57).

We now consider (61) inside the Knudsen layer, i.e., $\eta=O(1)$ or $\left(\boldsymbol{x}-\boldsymbol{x}_{\mathrm{w}}\right) \cdot \boldsymbol{n}=$ $O(\varepsilon)$. The $x$-dependence of $\hat{f}^{(0)}$ is through $\hat{\rho}, \hat{\boldsymbol{v}}$, and $\hat{T}$, the length scale of which is $O(1)$. Therefore, inside the Knudsen layer, they can be Taylor expanded around $\boldsymbol{x}=\boldsymbol{x}_{\mathrm{w}}$, that is,

$$
\hat{\rho}=\hat{\rho}_{\mathrm{B}}+O(\varepsilon \eta), \quad \hat{\boldsymbol{v}}=\hat{\boldsymbol{v}}_{\mathrm{B}}+O(\varepsilon \eta), \quad \hat{T}=\hat{T}_{\mathrm{B}}+O(\varepsilon \eta),
$$


where the subscript B indicates the value on the boundary $\boldsymbol{x}=\boldsymbol{x}_{\mathrm{w}}$ or $\eta=0$. Because $\hat{\boldsymbol{v}}_{\mathrm{B}}=\hat{\boldsymbol{v}}_{\mathrm{w}}+O(\varepsilon)$ and $\hat{T}_{\mathrm{B}}=\hat{T}_{\mathrm{w}}+O(\varepsilon)[(59)]$, we can write

$$
\hat{\rho}=\hat{\rho}_{\mathrm{B}}+O(\varepsilon \eta), \quad \hat{\boldsymbol{v}}=\hat{\boldsymbol{v}}_{\mathrm{w}}+O(\varepsilon(\eta+1)), \quad \hat{T}=\hat{T}_{\mathrm{w}}+O(\varepsilon(\eta+1)) .
$$

With these expressions, $\hat{f}^{(0)}$ inside the Knudsen layer is expanded as

$$
\hat{f}^{(0)}=\hat{f}_{\mathrm{w}}[1+O(\varepsilon(\eta+1))],
$$

where

$$
\hat{f}_{\mathrm{w}}=\frac{\hat{\rho}_{\mathrm{B}}}{\left(\pi \hat{T}_{\mathrm{w}}\right)^{3 / 2}} \exp \left(-\frac{\left|\boldsymbol{\zeta}-\hat{\boldsymbol{v}}_{\mathrm{w}}\right|^{2}}{\hat{T}_{\mathrm{w}}}\right)
$$

Therefore, the term $2 \hat{J}\left(\hat{f}^{(0)}, \hat{f}_{\mathrm{K}}^{(1)}\right)$ in (61) can be written as follows:

$$
2 \hat{J}\left(\hat{f}^{(0)}, \hat{f}_{\mathrm{K}}^{(1)}\right)=2 \hat{J}\left(\hat{f}_{\mathrm{w}}, \hat{f}_{\mathrm{K}}^{(1)}\right)+O\left(\varepsilon(\eta+1) \hat{f}_{\mathrm{K}}^{(1)}\right) .
$$

Note that $\eta \hat{f}_{\mathrm{K}}^{(1)} \rightarrow 0$ as $\eta \rightarrow \infty$ because of the rapid decay of $\hat{f}_{\mathrm{K}}^{(1)}$. Next we need to express the left-hand side of (61) in terms of the new variables $\left(\tilde{t}, \eta, \chi_{1}, \chi_{2}, \zeta_{\mathrm{w}}\right)$. From (64), we have

$$
\begin{aligned}
& \frac{\partial}{\partial \hat{t}}=\frac{\partial \tilde{t}}{\partial \hat{t}} \frac{\partial}{\partial \tilde{t}}+\frac{\partial \eta}{\partial \hat{t}} \frac{\partial}{\partial \eta}+\frac{\partial \chi_{1}}{\partial \hat{t}} \frac{\partial}{\partial \chi_{1}}+\frac{\partial \chi_{2}}{\partial \hat{t}} \frac{\partial}{\partial \chi_{2}}+\frac{\partial \zeta_{\mathrm{w} i}}{\partial \hat{t}} \frac{\partial}{\partial \zeta_{\mathrm{w} i}}, \\
& \frac{\partial}{\partial x_{i}}=\frac{\partial \tilde{t}}{\partial x_{i}} \frac{\partial}{\partial \tilde{t}}+\frac{\partial \eta}{\partial x_{i}} \frac{\partial}{\partial \eta}+\frac{\partial \chi_{1}}{\partial x_{i}} \frac{\partial}{\partial \chi_{1}}+\frac{\partial \chi_{2}}{\partial x_{i}} \frac{\partial}{\partial \chi_{2}}+\frac{\partial \zeta_{\mathrm{w} j}}{\partial x_{i}} \frac{\partial}{\partial \zeta_{\mathrm{w} j}} .
\end{aligned}
$$

The formula (64) gives the following expressions for the coefficients in (71) [see Appendix A]:

$$
\begin{aligned}
& \frac{\partial \tilde{t}}{\partial \hat{t}}=1, \quad \frac{\partial \eta}{\partial \hat{t}}=-\frac{1}{\varepsilon} \hat{v}_{\mathrm{w} i} n_{i}+O(1), \quad \frac{\partial \chi_{1,2}}{\partial \hat{t}}=O(1), \quad \frac{\partial \zeta_{\mathrm{w} i}}{\partial \hat{t}}=O(1) \\
& \frac{\partial \tilde{t}}{\partial x_{i}}=0, \quad \frac{\partial \eta}{\partial x_{i}}=\frac{1}{\varepsilon} n_{i}+O(1), \quad \frac{\partial \chi_{1,2}}{\partial x_{i}}=O(1), \quad \frac{\partial \zeta_{\mathrm{w} j}}{\partial x_{i}}=O(1) .
\end{aligned}
$$

If we substitute (70) and use (71) with (72) in (61), we obtain the following equation:

$$
\zeta_{\mathrm{w} i} n_{i} \frac{\partial \hat{f}_{\mathrm{K}}^{(1)}}{\partial \eta}=2 \hat{J}\left(\hat{f}_{\mathrm{w}}, \hat{f}_{\mathrm{K}}^{(1)}\right)+O(\varepsilon \mathscr{R})
$$

where $\mathscr{R}$ is a quantity of $O(1)$ that vanishes rapidly as $\eta \rightarrow \infty$ as well as $\left|\zeta_{\mathrm{w}}\right| \rightarrow \infty$.

Here, we should make the following comment. In the process of deriving (73), we have put the derivative term $\partial \hat{f}_{\mathrm{K}}^{(1)} / \partial \zeta_{\mathrm{w} i}$ in $O(\varepsilon \mathscr{R})$ term, assuming that it is of $O(1)$. We will eventually neglect the $O(\varepsilon \mathscr{R})$ term in (73) and obtain $\hat{f}_{\mathrm{K}}^{(1)}$ in the following sections. However, $\hat{f}_{\mathrm{K}}^{(1)}$ thus obtained has a singularity at $\zeta_{\mathrm{w} i} n_{i}=0$ on the boundary $\eta=0$, 
which originates from the discontinuity between the velocity distribution function for incident molecules and that for reflected molecules on the boundary, and the derivative $\partial \hat{f}_{\mathrm{K}}^{(1)} / \partial \zeta_{\mathrm{w} i}$ diverges there $[17,25,26]$. This means that we cannot neglect this term in $O(\varepsilon \mathscr{R})$ in (73) at the point $\zeta_{\mathrm{w} i} n_{i}=0, \eta=0$. However, it happens only locally at this point, and the rate of divergence is weak $\left(\approx \ln \left|\zeta_{\mathrm{w} i} n_{i}\right|\right.$ for $\left.\zeta_{\mathrm{w} i} n_{i} \rightarrow 0-\right)$. In fact, if we take any moment of (73) with respect to $\zeta_{\mathrm{w}}$, the corresponding moment of the $O(\varepsilon \mathscr{R})$ term remains of $O(\varepsilon)$. Therefore, this singularity is not likely to affect the slip boundary conditions derived through the analysis of $\hat{f}_{\mathrm{K}}^{(1)}$. The rigorous proof of this statement, which is a very hard mathematical problem, has been addressed in a recent paper [29] for a transport equation much simpler than the linearized Boltzmann equation.

Now, let us introduce the following $\mathscr{C}_{\mathrm{w}}$, its normal component $\mathscr{C}_{\mathrm{wn}}$, and its magnitude $\mathscr{C}_{\mathrm{w}}$ :

$$
\mathscr{C}_{\mathrm{w}}=\frac{\boldsymbol{\zeta}_{\mathrm{w}}}{\hat{T}_{\mathrm{w}}^{1 / 2}}=\frac{\boldsymbol{\zeta}-\hat{\boldsymbol{v}}_{\mathrm{w}}}{\hat{T}_{\mathrm{w}}^{1 / 2}}, \quad \mathscr{C}_{\mathrm{wn}}=\mathscr{C}_{\mathrm{w}} \cdot \boldsymbol{n}, \quad \mathscr{C}_{\mathrm{w}}=\left(\mathscr{C}_{\mathrm{w} j}^{2}\right)^{1 / 2}=\left|\mathscr{C}_{\mathrm{w}}\right|
$$

Then, $\hat{f}_{\mathrm{w}}$ can be expressed, using the function $E$ defined in (26), as

$$
\hat{f}_{\mathrm{w}}=\frac{\hat{\rho}_{\mathrm{B}}}{\hat{T}_{\mathrm{w}}^{3 / 2}} E\left(\mathscr{C}_{\mathrm{w}}\right)
$$

Note that $\hat{\rho}_{\mathrm{B}}$ and $\hat{T}_{\mathrm{w}}$ are functions of $\left(\tilde{t}, \chi_{1}, \chi_{2}\right)$. If we let

$$
\hat{f}_{\mathrm{K}}^{(1)}\left(\tilde{t}, \eta, \chi_{1}, \chi_{2}, \hat{T}_{\mathrm{w}}^{1 / 2} \mathscr{C}_{\mathrm{w}}\right)=\hat{f}_{\mathrm{w}} \Phi\left(\tilde{t}, \eta, \chi_{1}, \chi_{2}, \mathscr{C}_{\mathrm{w}}\right),
$$

change the velocity variable from $\zeta_{\mathrm{w}}$ to $\mathscr{C}_{\mathrm{w}}$, and recall (31), then $2 \hat{J}\left(\hat{f}_{\mathrm{w}}, \hat{f}_{\mathrm{K}}^{(1)}\right)$ in (73) can be transformed as

$$
2 \hat{J}\left(\hat{f}_{\mathrm{w}}, \hat{f}_{\mathrm{K}}^{(1)}\right)=2 \hat{J}\left(\hat{f}_{\mathrm{w}}, \hat{f}_{\mathrm{w}} \Phi\right)=\frac{\hat{\rho}_{\mathrm{B}}^{2}}{\hat{T}_{\mathrm{w}}} E\left(\mathscr{C}_{\mathrm{w}}\right) \mathscr{L}_{\hat{T}_{\mathrm{w}}}(\Phi)\left(\tilde{t}, \eta, \chi_{1}, \chi_{2}, \mathscr{C}_{\mathrm{w}}\right),
$$

where the last parentheses indicate the independent variables of $\mathscr{L}_{\hat{T}_{\mathrm{w}}}(\Phi)$ explicitly. In consequence, we have the following expression of (73):

$$
\mathscr{C}_{\mathrm{wn}} \frac{\partial \Phi}{\partial \eta}=\hat{\rho}_{\mathrm{B}} \mathscr{L}_{\hat{T}_{\mathrm{w}}}(\Phi)+O\left(\varepsilon \mathscr{R} / E\left(\mathscr{C}_{\mathrm{w}}\right)\right) .
$$

In order to get rid of $\hat{\rho}_{\mathrm{B}}$ in (78), we further introduce the new normal coordinate $y$ in place of $\eta$,

$$
y=\hat{\rho}_{\mathrm{B}} \eta,
$$

and let

$$
\Phi\left(\tilde{t}, \hat{\rho}_{\mathrm{B}}^{-1} y, \chi_{1}, \chi_{2}, \mathscr{C}_{\mathrm{w}}\right)=\phi\left(\tilde{t}, y, \chi_{1}, \chi_{2}, \mathscr{C}_{\mathrm{w}}\right)
$$


Then, (78) reduces to

$$
\mathscr{C}_{\mathrm{wn}} \frac{\partial \phi}{\partial y}=\mathscr{L}_{\hat{T}_{\mathrm{w}}}(\phi)+O\left(\varepsilon \mathscr{R} / E\left(\mathscr{C}_{\mathrm{w}}\right)\right)
$$

If we neglect the terms of $O\left(\varepsilon \mathscr{R} / E\left(\mathscr{C}_{\mathrm{w}}\right)\right)$, we obtain the equation for $\phi$, i.e., that for $\hat{f}_{\mathrm{K}}^{(1)}$.

If we use (75), (76), and (80) and the expansion of the Chapman-Enskog macroscopic quantities near the boundary (67) in (60), we can obtain the following expression of the Knudsen-layer corrections of the macroscopic quantities:

$$
\begin{aligned}
& \frac{\hat{\rho}_{\mathrm{K}}^{(1)}}{\hat{\rho}_{\mathrm{B}}}=\int \phi E\left(\mathscr{C}_{\mathrm{w}}\right) d \mathscr{C}_{\mathrm{w}}, \\
& \frac{\hat{v}_{\mathrm{K} i}^{(1)}}{\hat{T}_{\mathrm{w}}^{1 / 2}}=\int \mathscr{C}_{\mathrm{w} i} \phi E\left(\mathscr{C}_{\mathrm{w}}\right) d \mathscr{C}_{\mathrm{w}}+O(\varepsilon(y+1)), \\
& \frac{\hat{T}_{\mathrm{K}}^{(1)}}{\hat{T}_{\mathrm{w}}}=\frac{2}{3} \int\left(\mathscr{C}_{\mathrm{w}}^{2}-\frac{3}{2}\right) \phi E\left(\mathscr{C}_{\mathrm{w}}\right) d \mathscr{C}_{\mathrm{w}}+O(\varepsilon(y+1)), \\
& \hat{p}_{\mathrm{K}}^{(1)}=\hat{\rho}_{\mathrm{B}} \hat{T}_{\mathrm{K}}^{(1)}+\hat{\rho}_{\mathrm{K}}^{(1)} \hat{T}_{\mathrm{w}}+O(\varepsilon(y+1)), \\
& \frac{\hat{p}_{\mathrm{K} i j}^{(1)}}{\hat{\rho}_{\mathrm{B}} \hat{T}_{\mathrm{w}}}=2 \int \mathscr{C}_{\mathrm{w} i} \mathscr{C}_{\mathrm{w} j} \phi E\left(\mathscr{C}_{\mathrm{w}}\right) d \mathscr{C}_{\mathrm{w}}+O(\varepsilon(y+1)), \\
& \frac{\hat{q}_{\mathrm{K} i}^{(1)}}{\hat{\rho}_{\mathrm{B}} \hat{T}_{\mathrm{w}}^{3 / 2}}=\int \mathscr{C}_{\mathrm{w} i}\left(\mathscr{C}_{\mathrm{w}}^{2}-\frac{5}{2}\right) \phi E\left(\mathscr{C}_{\mathrm{w}}\right) d \mathscr{C}_{\mathrm{w}}+O(\varepsilon(y+1)) .
\end{aligned}
$$

For the BGK model, we take the same procedure as that led to (61). In this process, we note that $\hat{f}_{e}$ with $\hat{f}=\hat{f}_{\text {tot }}$, which we denote by $\left(\hat{f}_{e}\right)_{\text {tot }}$, is the $\hat{f}_{e}$ with $\hat{\rho}=\hat{\rho}_{\text {tot }}, \hat{\boldsymbol{v}}=\hat{\boldsymbol{v}}_{\text {tot }}$, and $\hat{T}=\hat{T}_{\text {tot }}$. Therefore, by using (56) and (58), we can expand $\left(\hat{f}_{e}\right)_{\text {tot }}$ as follows:

$$
\begin{aligned}
\left(\hat{f}_{e}\right)_{\text {tot }}=\left(\hat{f}_{e}\right)_{\mathrm{CE}}\{1+ & {\left[\frac{\hat{\rho}_{\mathrm{K}}^{(1)}}{\hat{\rho}}+2 \frac{(\boldsymbol{\zeta}-\hat{\boldsymbol{v}}) \cdot \hat{\boldsymbol{v}}_{\mathrm{K}}^{(1)}}{\hat{T}}\right.} \\
& \left.\left.+\frac{1}{\hat{T}}\left(\frac{|\boldsymbol{\zeta}-\hat{\boldsymbol{v}}|^{2}}{\hat{T}}-\frac{3}{2}\right) \hat{T}_{\mathrm{K}}^{(1)}\right] \varepsilon+O\left(\varepsilon^{2} R_{h}\right)\right\},
\end{aligned}
$$

where $\left(\hat{f}_{e}\right)_{\text {CE }}$ denotes $\hat{f}_{e}$ with $\hat{f}=\hat{f}_{\text {CE }}$ and is nothing but $\hat{f}^{(0)}$. Using this relation, we obtain (61) with $2 \hat{J}\left(\hat{f}^{(0)}, \hat{f}_{\mathrm{K}}^{(1)}\right)$ replaced by

$$
\hat{A}_{c}(\hat{T}) \hat{\rho}\left\{\hat{f}^{(0)}\left[\frac{\hat{\rho}_{\mathrm{K}}^{(1)}}{\hat{\rho}}+2 \frac{(\boldsymbol{\zeta}-\hat{\boldsymbol{v}}) \cdot \hat{\boldsymbol{v}}_{\mathrm{K}}^{(1)}}{\hat{T}}+\frac{1}{\hat{T}}\left(\frac{|\boldsymbol{\zeta}-\hat{\boldsymbol{v}}|^{2}}{\hat{T}}-\frac{3}{2}\right) \hat{T}_{\mathrm{K}}^{(1)}\right]-\hat{f}_{\mathrm{K}}^{(1)}\right\} .
$$

Then, we take the same procedure as that led to (81). That is, we use the expansions (67) and (68) and (75), (76), and (80) as well as the necessary change of variables. As the result, we obtain (81) with $\mathscr{L}_{\hat{T}_{\mathrm{w}}}(\phi)$ replaced by $\mathscr{L}_{\hat{T}_{\mathrm{w}} \mathrm{BGK}}(\phi)$, where $\mathscr{L}_{a \mathrm{BGK}}(\cdot)$ is defined in (33). However, because $\hat{A}_{c}(a) / \sqrt{a}$ is factored out in the form of $\mathscr{L}_{a \mathrm{BGK}}(\cdot)$ 
[cf. (33a)], we can get rid of $\hat{T}_{\mathrm{w}}$ from the equation by defining $y$ and $\phi$ as follows, in place of (79) and (80):

$$
\begin{aligned}
& y=\left[\hat{\rho}_{\mathrm{B}} \hat{A}_{c}\left(\hat{T}_{\mathrm{w}}\right) / \hat{T}_{\mathrm{w}}^{1 / 2}\right] \eta, \\
& \Phi\left(\tilde{t},\left[\hat{T}_{\mathrm{w}}^{1 / 2} / \hat{\rho}_{\mathrm{B}} \hat{A}_{c}\left(\hat{T}_{\mathrm{w}}\right)\right] y, \chi_{1}, \chi_{2}, \mathscr{C}_{\mathrm{w}}\right)=\phi\left(\tilde{t}, y, \chi_{1}, \chi_{2}, \mathscr{C}_{\mathrm{w}}\right) .
\end{aligned}
$$

In consequence, we obtain the equation corresponding to (81), i.e.,

$$
\mathscr{C}_{\mathrm{wn}} \frac{\partial \phi}{\partial y}=\mathscr{L}_{\mathrm{BGK}}(\phi)+O\left(\varepsilon \mathscr{R} / E\left(\mathscr{C}_{\mathrm{w}}\right)\right)
$$

where $\mathscr{L}_{\mathrm{BGK}}(\cdot)$ is defined in (33).

\subsubsection{Knudsen-Layer Boundary Condition}

Now we consider the boundary condition. The total solution $\hat{f}_{\text {tot }}[(55)]$ should satisfy the boundary condition (27). If we insert (55) with $\hat{f}_{\mathrm{CE}}$ given by (39) and with $\hat{f}_{\mathrm{K}}$ given by (57) in (27), we obtain, at $\eta=0$ (or $\boldsymbol{x}=\boldsymbol{x}_{\mathrm{w}}$ ),

$$
\begin{aligned}
& \varepsilon \hat{f}_{\mathrm{K}}^{(1)}=(1-\alpha) \varepsilon \hat{\mathrm{R}} \hat{f}_{\mathrm{K}}^{(1)}-\hat{f}^{(0)}-\varepsilon \hat{f}^{(1)} \\
&+(1-\alpha) \hat{\mathrm{R}}\left(\hat{f}^{(0)}+\varepsilon \hat{f}^{(1)}\right)+\alpha \frac{\hat{\rho}_{\mathrm{W}}}{\hat{\rho}_{\mathrm{B}}} \hat{f}_{\mathrm{w}}+O\left(\varepsilon^{2}\right), \text { for } \quad\left(\boldsymbol{\zeta}-\hat{\boldsymbol{v}}_{\mathrm{w}}\right) \cdot \boldsymbol{n}>0, \\
& \hat{\rho}_{\mathrm{w}}=-2\left(\frac{\pi}{\hat{T}_{\mathrm{w}}}\right)^{1 / 2} \int_{\left(\boldsymbol{\zeta}-\hat{\boldsymbol{v}}_{\mathrm{W}}\right) \cdot \boldsymbol{n}<0}\left(\boldsymbol{\zeta}-\hat{\boldsymbol{v}}_{\mathrm{w}}\right) \cdot \boldsymbol{n}\left(\hat{f}^{(0)}+\varepsilon \hat{f}^{(1)}+\varepsilon \hat{f}_{\mathrm{K}}^{(1)}\right) d \boldsymbol{\zeta}+O\left(\varepsilon^{2}\right) .
\end{aligned}
$$

As shown in Appendix B, $\hat{f}^{(0)}, \hat{f}^{(1)}$, and $\hat{\rho}_{\mathrm{w}}$ in (87) can be expressed in the following form:

$$
\begin{aligned}
\hat{f}^{(0)}=\hat{f}_{\mathrm{w}}\left\{1+\varepsilon\left[2 \mathscr{C}_{\mathrm{w} j} \frac{\check{v}_{j}}{\hat{T}_{\mathrm{w}}^{1 / 2}}+\left(\mathscr{C}_{\mathrm{w}}^{2}-\frac{3}{2}\right) \frac{\check{T}}{\hat{T}_{\mathrm{w}}}\right]+O\left(\varepsilon^{2}\right)\right\} \\
\hat{f}^{(1)}=\hat{f}_{\mathrm{w}}\left[\Psi_{\mathrm{w}}+O(\varepsilon)\right] \\
\frac{\hat{\rho}_{\mathrm{w}}}{\hat{\rho}_{\mathrm{B}}}=1+\varepsilon\left\{-\sqrt{\pi} \frac{\check{v}_{i}}{\hat{T}_{\mathrm{w}}^{1 / 2}} n_{i}+\frac{1}{2} \frac{\check{T}}{\hat{T}_{\mathrm{w}}}\right. \\
\quad-\frac{1}{6} \mathscr{I}_{\mathscr{B}} \frac{1}{\hat{\rho}_{\mathrm{B}} \hat{T}_{\mathrm{w}}^{1 / 2}}\left[\left(\frac{\partial \hat{v}_{j}}{\partial x_{i}}\right)_{\mathrm{B}}+\left(\frac{\partial \hat{v}_{i}}{\partial x_{j}}\right)_{\mathrm{B}}\right]\left[n_{i} n_{j}-\frac{1}{2}\left(\delta_{i j}-n_{i} n_{j}\right)\right] \\
\left.\quad-\frac{2}{\hat{\rho}_{\mathrm{B}}}\left(\frac{\pi}{\hat{T}_{\mathrm{w}}}\right)^{1 / 2} \int_{\left(\zeta-\hat{\boldsymbol{v}}_{\mathrm{w}}\right) \cdot n<0}\left(\zeta-\hat{\boldsymbol{v}}_{\mathrm{w}}\right) \cdot \boldsymbol{n} \hat{f}_{\mathrm{K}}^{(1)} d \zeta\right\}+O\left(\varepsilon^{2}\right)
\end{aligned}
$$

where

$$
\begin{aligned}
\Psi_{\mathrm{w}}= & -\frac{1}{\hat{\rho}_{\mathrm{B}} \hat{T}_{\mathrm{w}}} \mathscr{C}_{\mathrm{w} i} \mathscr{A}\left(\mathscr{C}_{\mathrm{w}}, \hat{T}_{\mathrm{w}}\right)\left(\frac{\partial \hat{T}}{\partial x_{i}}\right)_{\mathrm{B}} \\
& -\frac{1}{2 \hat{\rho}_{\mathrm{B}} \hat{T}_{\mathrm{w}}^{1 / 2}}\left(\mathscr{C}_{\mathrm{w} i} \mathscr{C}_{\mathrm{w} j}-\frac{1}{3} \mathscr{C}_{\mathrm{w}}^{2} \delta_{i j}\right) \mathscr{B}^{(0)}\left(\mathscr{C}_{\mathrm{w}}, \hat{T}_{\mathrm{w}}\right)\left[\left(\frac{\partial \hat{v}_{j}}{\partial x_{i}}\right)_{\mathrm{B}}+\left(\frac{\partial \hat{v}_{i}}{\partial x_{j}}\right)_{\mathrm{B}}\right],
\end{aligned}
$$




$$
\mathscr{I}_{\mathscr{B}}=\int_{0}^{\infty} \mathscr{C}_{\mathrm{w}}^{5} \mathscr{B}^{(0)}\left(\mathscr{C}_{\mathrm{w}}, \hat{T}_{\mathrm{w}}\right) \exp \left(-\mathscr{C}_{\mathrm{w}}^{2}\right) d \mathscr{C}_{\mathrm{w}}
$$

For the new velocity variable $\mathscr{C}_{\mathrm{w}}$, the reflection operator (28) should be replaced with the following $\widetilde{R}$ :

$$
\widetilde{\mathrm{R}} \hat{g}\left(\mathscr{C}_{\mathrm{w} i}\right)=\hat{g}\left(\mathscr{C}_{\mathrm{w} i}-2 \mathscr{C}_{\mathrm{w} j} n_{j} n_{i}\right)
$$

where $\hat{g}\left(\mathscr{C}_{\mathrm{w} i}\right)$ is a function of $\mathscr{C}_{\mathrm{w} i}$. Note that $\widetilde{\mathrm{R}} \hat{\mathrm{w}}_{\mathrm{w}}\left(\mathscr{C}_{\mathrm{w}}\right)=\hat{f}_{\mathrm{w}}\left(\mathscr{C}_{\mathrm{w}}\right)$. Then, we can write (87) in the following intermediate form:

$$
\begin{aligned}
\hat{f}_{\mathrm{K}}^{(1)}= & (1-\alpha) \widetilde{\mathrm{R}} \hat{\mathrm{K}}_{\mathrm{K}}^{(1)}+\hat{f}_{\mathrm{w}}[\hat{\mathscr{F}}+O(\varepsilon)], \quad \text { at } \eta=0, \text { for }\left(\boldsymbol{\zeta}-\hat{\boldsymbol{v}}_{\mathrm{w}}\right) \cdot \boldsymbol{n}>0, \\
\hat{\mathscr{F}}= & -2\left[\mathscr{C}_{\mathrm{w} j}-(1-\alpha) \widetilde{\mathrm{R}} \mathscr{C}_{\mathrm{w} j}\right] \frac{\check{v}_{j}}{\hat{T}_{\mathrm{w}}^{1 / 2}}-\alpha \sqrt{\pi} \frac{\check{v}_{j}}{\hat{T}_{\mathrm{w}}^{1 / 2}} n_{j}-\alpha\left(\mathscr{C}_{\mathrm{w}}^{2}-2\right) \frac{\check{T}_{\mathrm{w}}}{\hat{T}_{\mathrm{w}}} \\
& -\Psi_{\mathrm{w}}+(1-\alpha) \widetilde{\mathrm{R}} \Psi_{\mathrm{w}} \\
& -\alpha\left\{\frac{1}{6} \mathscr{I}_{\mathscr{B}} \frac{1}{\hat{\rho}_{\mathrm{B}} \hat{T}_{\mathrm{w}}^{1 / 2}}\left[\left(\frac{\partial \hat{v}_{j}}{\partial x_{i}}\right)_{\mathrm{B}}+\left(\frac{\partial \hat{v}_{i}}{\partial x_{j}}\right)_{\mathrm{B}}\right]\left[n_{i} n_{j}-\frac{1}{2}\left(\delta_{i j}-n_{i} n_{j}\right)\right]\right. \\
& \left.+\frac{2}{\hat{\rho}_{\mathrm{B}}}\left(\frac{\pi}{\hat{T}_{\mathrm{w}}}\right)^{1 / 2} \int_{\left(\boldsymbol{\zeta}-\hat{\boldsymbol{v}}_{\mathrm{w}}\right) \cdot \boldsymbol{n}<0}\left(\boldsymbol{\zeta}-\hat{\boldsymbol{v}}_{\mathrm{w}}\right) \cdot \boldsymbol{n} \hat{f}_{\mathrm{K}}^{(1)} d \zeta\right\} .
\end{aligned}
$$

We can simplify (91) a little more, as shown below.

If we integrate (73) over the whole space of $\zeta_{\mathrm{w}}$ noting that the both sides are the functions of $\left(\tilde{t}, \eta, \chi_{1}, \chi_{2}, \zeta_{\mathrm{w}}\right)$, we have

$$
\frac{\partial}{\partial \eta} \int \zeta_{\mathrm{w} i} n_{i} \hat{f}_{\mathrm{K}}^{(1)} d \zeta_{\mathrm{w}}=O\left(\varepsilon \int \mathscr{R} d \zeta_{\mathrm{w}}\right)
$$

because of the property of $\hat{J}(\cdot, \cdot)$. Since $\hat{f}_{\mathrm{K}}^{(1)}$ and $\mathscr{R}$ vanish rapidly as $\eta \rightarrow \infty$, the integration of the above equation with respect to $\eta$ from 0 to $\infty$ leads to $\int \zeta_{\mathrm{w} i} n_{i} \hat{f}_{\mathrm{K}}^{(1)} d \zeta_{\mathrm{w}}=$ $O(\varepsilon)$ at $\eta=0$. This implies that

$$
\int \zeta_{\mathrm{w} i} n_{i} \hat{f}_{\mathrm{K}}^{(1)} d \zeta_{\mathrm{w}}=O(\varepsilon), \text { for any } \eta
$$

On the other hand, the condition of no net mass flow on the boundary, (29), should be satisfied by $\hat{f}_{\text {tot }}$ of (55). Substituting (55) into (29) and taking the properties of $\hat{f}_{\mathrm{CE}}$ into account, we have $\hat{\rho}_{\mathrm{B}}\left(\hat{\boldsymbol{v}}_{\mathrm{B}}-\hat{\boldsymbol{v}}_{\mathrm{w}}\right) \cdot \boldsymbol{n}+\int\left(\boldsymbol{\zeta}-\hat{\boldsymbol{v}}_{\mathrm{w}}\right) \cdot \boldsymbol{n} \hat{f}_{\mathrm{K}}(\boldsymbol{\eta}=0) d \boldsymbol{\zeta}=0$. Making use of (57), (59), (64c), and (93), we can show that

$$
\hat{\rho}_{\mathrm{B}} \varepsilon \check{\boldsymbol{v}} \cdot \boldsymbol{n}=-\int \boldsymbol{\zeta}_{\mathrm{w}} \cdot \boldsymbol{n}\left[\varepsilon \hat{f}_{\mathrm{K}}^{(1)}+O\left(\varepsilon^{2} R_{f}\right)\right](\eta=0) d \boldsymbol{\zeta}_{\mathrm{w}}=O\left(\varepsilon^{2}\right),
$$

that is,

$$
\check{\boldsymbol{v}} \cdot \boldsymbol{n}=O(\varepsilon)
$$


Next, we consider $\left[\left(\partial \hat{v}_{i} / \partial x_{j}\right)_{\mathrm{B}}+\left(\partial \hat{v}_{j} / \partial x_{i}\right)_{\mathrm{B}}\right]\left(\delta_{i j}-n_{i} n_{j}\right)$ and $\left[\left(\partial \hat{v}_{i} / \partial x_{j}\right)_{\mathrm{B}}+\left(\partial \hat{v}_{j} / \partial x_{i}\right)_{\mathrm{B}}\right]\left(\delta_{i k}-\right.$ $\left.n_{i} n_{k}\right)\left(\delta_{j l}-n_{j} n_{l}\right)$. Since the derivatives in these expressions are all in the tangential directions to the boundary [note that $a_{i}\left(\delta_{i j}-n_{i} n_{j}\right)=a_{j}-\left(a_{i} n_{i}\right) n_{j}$ is a tangential vector], we can replace $\hat{\boldsymbol{v}}$ inside the derivatives with $\hat{\boldsymbol{v}}_{\mathrm{B}}$, which is equal to $\hat{\boldsymbol{v}}_{\mathrm{W}}+\varepsilon \check{\boldsymbol{v}}$ [(59)]. On the other hand, $\hat{\boldsymbol{v}}_{\mathrm{w}}$ is the velocity of rigid-body motion, so that the relation $\partial \hat{v}_{\mathrm{w} i} / \partial x_{j}+\partial \hat{v}_{\mathrm{w} j} / \partial x_{i}=0$ holds for any $i$ and $j$. Therefore, we have the following relations:

$$
\begin{gathered}
{\left[\left(\frac{\partial \hat{v}_{i}}{\partial x_{j}}\right)_{\mathrm{B}}+\left(\frac{\partial \hat{v}_{j}}{\partial x_{i}}\right)_{\mathrm{B}}\right]\left(\delta_{i j}-n_{i} n_{j}\right)=\varepsilon\left(\frac{\partial \check{v}_{i}}{\partial x_{j}}+\frac{\partial \check{v}_{j}}{\partial x_{i}}\right)\left(\delta_{i j}-n_{i} n_{j}\right)=O(\varepsilon),} \\
{\left[\left(\frac{\partial \hat{v}_{i}}{\partial x_{j}}\right)_{\mathrm{B}}+\left(\frac{\partial \hat{v}_{j}}{\partial x_{i}}\right)_{\mathrm{B}}\right]\left(\delta_{i k}-n_{i} n_{k}\right)\left(\delta_{j l}-n_{j} n_{l}\right)} \\
=\varepsilon\left(\frac{\partial \check{v}_{i}}{\partial x_{j}}+\frac{\partial \check{v}_{j}}{\partial x_{i}}\right)\left(\delta_{i k}-n_{i} n_{k}\right)\left(\delta_{j l}-n_{j} n_{l}\right)=O(\varepsilon) .
\end{gathered}
$$

In consequence of (96), the following relation holds:

$$
\begin{aligned}
\left(\mathscr{C}_{\mathrm{w} i} \mathscr{C}_{\mathrm{w} j}-\frac{1}{3} \mathscr{C}_{\mathrm{w}}^{2} \delta_{i j}\right)\left[\left(\frac{\partial \hat{v}_{j}}{\partial x_{i}}\right)_{\mathrm{B}}+\left(\frac{\partial \hat{v}_{i}}{\partial x_{j}}\right)_{\mathrm{B}}\right] \\
=2\left(\mathscr{C}_{\mathrm{wn}}^{2}-\frac{1}{3} \mathscr{C}_{\mathrm{w}}^{2}\right)\left(\frac{\partial \hat{v}_{i}}{\partial x_{j}}\right)_{\mathrm{B}} n_{i} n_{j} \\
\quad+2 \mathscr{C}_{\mathrm{wn}} \mathscr{C}_{\mathrm{w} l} n_{i}\left(\delta_{j l}-n_{j} n_{l}\right)\left[\left(\frac{\partial \hat{v}_{j}}{\partial x_{i}}\right)_{\mathrm{B}}+\left(\frac{\partial \hat{v}_{i}}{\partial x_{j}}\right)_{\mathrm{B}}\right]+O(\varepsilon) .
\end{aligned}
$$

Now we use (76) and (80) in (91) to derive the boundary condition for $\phi$. More specifically, we change the variable of integration from $\zeta$ to $\mathscr{C}_{\mathrm{w}}$, using (74) and (75), in the integral in (91b) and take into account (95) and (97) and the relations $\mathscr{C}_{\mathrm{w} i}=$ $\mathscr{C}_{\mathrm{wn}} n_{i}+\mathscr{C}_{\mathrm{w} j}\left(\delta_{i j}-n_{i} n_{j}\right)$ and $\widetilde{\mathrm{R}} \mathscr{C}_{\mathrm{w} i}=-\mathscr{C}_{\mathrm{wn}} n_{i}+\mathscr{C}_{\mathrm{w} j}\left(\delta_{i j}-n_{i} n_{j}\right)$. Then, (91) reduces to the following form:

$$
\begin{aligned}
\phi= & (1-\alpha) \widetilde{\mathrm{R}} \phi-\alpha\left(\mathscr{C}_{\mathrm{w}}^{2}-2\right) \frac{\check{T}}{\hat{T}_{\mathrm{w}}}-2 \alpha \mathscr{C}_{\mathrm{w} i}\left(\delta_{i j}-n_{i} n_{j}\right) \frac{\check{v}_{j}}{\hat{T}_{\mathrm{w}}^{1 / 2}} \\
& -2 \alpha \sqrt{\pi} \int_{\mathscr{C}_{\mathrm{wn}}<0} \mathscr{C}_{\mathrm{wn}} \phi E\left(\mathscr{C}_{\mathrm{w}}\right) d \mathscr{C}_{\mathrm{w}}+(2-\alpha) \frac{1}{\hat{\rho}_{\mathrm{B}} \hat{T}_{\mathrm{w}}} \mathscr{C}_{\mathrm{wn}} \mathscr{A}\left(\mathscr{C}_{\mathrm{w}}, \hat{T}_{\mathrm{w}}\right)\left(\frac{\partial \hat{T}}{\partial x_{i}}\right)_{\mathrm{B}} n_{i} \\
& +\alpha\left[\left(\mathscr{C}_{\mathrm{wn}}^{2}-\frac{1}{3} \mathscr{C}_{\mathrm{w}}^{2}\right) \mathscr{B}^{(0)}\left(\mathscr{C}_{\mathrm{w}}, \hat{T}_{\mathrm{w}}\right)-\frac{1}{3} \mathscr{I}_{\mathscr{B}}\right] \frac{1}{\hat{\rho}_{\mathrm{B}} \hat{T}_{\mathrm{w}}^{1 / 2}}\left(\frac{\partial \hat{v}_{i}}{\partial x_{j}}\right)_{\mathrm{B}} n_{i} n_{j} \\
& +(2-\alpha) \frac{1}{\hat{\rho}_{\mathrm{B}} \hat{T}_{\mathrm{w}}^{1 / 2}} \mathscr{C}_{\mathrm{wn}} \mathscr{C}_{\mathrm{w} l} n_{i}\left(\delta_{j l}-n_{j} n_{l}\right) \mathscr{B}^{(0)}\left(\mathscr{C}_{\mathrm{w}}, \hat{T}_{\mathrm{w}}\right)\left[\left(\frac{\partial \hat{v}_{j}}{\partial x_{i}}\right)_{\mathrm{B}}+\left(\frac{\partial \hat{v}_{i}}{\partial x_{j}}\right)_{\mathrm{B}}\right] \\
& +\alpha \frac{1}{\hat{\rho}_{\mathrm{B}} \hat{T}_{\mathrm{w}}} \mathscr{C}_{\mathrm{w} i}\left(\delta_{i j}-n_{i} n_{j}\right) \mathscr{A}\left(\mathscr{C}_{\mathrm{w}}, \hat{T}_{\mathrm{w}}\right)\left(\frac{\partial \hat{T}}{\partial x_{j}}\right)_{\mathrm{B}}+O(\varepsilon), \quad\left(y=0, \mathscr{C}_{\mathrm{wn}}>0\right) .
\end{aligned}
$$




\subsubsection{Summary and Remarks}

Now we omit the terms of $O(\varepsilon)$ in (81) and (98) and take into account (65b). Then, we obtain the following boundary-value problem in the half space $(y>0)$ for $\phi$. In order to avoid cumbersome notations and to match the variables with those in the definition of the linearized collision operators in (30), (31), and (33), we just change the name of the variable from $\mathscr{C}_{\mathrm{w}}$ to $\zeta$ and denote $\phi$ as a function of $\left(\tilde{t}, y, \chi_{1}, \chi_{2}, \zeta\right)$, i.e.,

$$
\mathscr{C}_{\mathrm{w}} \rightarrow \boldsymbol{\zeta}, \quad \phi\left(\tilde{t}, y, \chi_{1}, \chi_{2}, \mathscr{C}_{\mathrm{w}}\right) \rightarrow \phi\left(\tilde{t}, y, \chi_{1}, \chi_{2}, \boldsymbol{\zeta}\right)
$$

Then, the problem for $\phi$ becomes as follows:

$$
\begin{aligned}
\zeta_{\mathrm{n}} & \frac{\partial \phi}{\partial y}=\mathscr{L}_{\hat{T}_{\mathrm{w}}}(\phi), \quad(y>0), \\
\phi= & (1-\alpha) \widetilde{\mathrm{R}} \phi-\alpha\left(\zeta^{2}-2\right) \frac{\check{T}}{\hat{T}_{\mathrm{w}}}-2 \alpha \zeta_{i}\left(\delta_{i j}-n_{i} n_{j}\right) \frac{\check{v}_{j}}{\hat{T}_{\mathrm{w}}^{1 / 2}} \\
& -2 \alpha \sqrt{\pi} \int_{\zeta_{\mathrm{n}}<0} \zeta_{\mathrm{n}} \phi E(\zeta) d \zeta+(2-\alpha) \frac{1}{\hat{\rho}_{\mathrm{B}} \hat{T}_{\mathrm{w}}} \zeta_{\mathrm{n}} \mathscr{A}\left(\zeta, \hat{T}_{\mathrm{w}}\right)\left(\frac{\partial \hat{T}}{\partial x_{i}}\right)_{\mathrm{B}} n_{i} \\
& +\alpha\left[\left(\zeta_{\mathrm{n}}^{2}-\frac{1}{3} \zeta^{2}\right) \mathscr{B}^{(0)}\left(\zeta, \hat{T}_{\mathrm{w}}\right)-\frac{1}{3} \mathscr{I}_{\mathscr{B}}\right] \frac{1}{\hat{\rho}_{\mathrm{B}} \hat{T}_{\mathrm{w}}^{1 / 2}}\left(\frac{\partial \hat{v}_{i}}{\partial x_{j}}\right)_{\mathrm{B}} n_{i} n_{j} \\
& +(2-\alpha) \frac{1}{\hat{\rho}_{\mathrm{B}} \hat{T}_{\mathrm{w}}^{1 / 2}} \zeta_{\mathrm{n}} \zeta_{l} n_{i}\left(\delta_{j l}-n_{j} n_{l}\right) \mathscr{B}^{(0)}\left(\zeta, \hat{T}_{\mathrm{w}}\right)\left[\left(\frac{\partial \hat{v}_{j}}{\partial x_{i}}\right)_{\mathrm{B}}+\left(\frac{\partial \hat{v}_{i}}{\partial x_{j}}\right)_{\mathrm{B}}\right] \\
& +\alpha \frac{1}{\hat{\rho}_{\mathrm{B}} \hat{T}_{\mathrm{w}}} \zeta_{i}\left(\delta_{i j}-n_{i} n_{j}\right) \mathscr{A}\left(\zeta, \hat{T}_{\mathrm{w}}\right)\left(\frac{\partial \hat{T}}{\partial x_{j}}\right)_{\mathrm{B}}, \quad\left(y=0, \zeta_{\mathrm{n}}>0\right), \\
\phi \rightarrow & 0, \quad(y \rightarrow \infty) .
\end{aligned}
$$

For the BGK model, (100a) is replaced by [cf. (86)]

$$
\zeta_{\mathrm{n}} \frac{\partial \phi}{\partial y}=\mathscr{L}_{\mathrm{BGK}}(\phi), \quad(y>0)
$$

and (79) and (80) are replaced by (85).

The Knudsen-layer problem, (100), is equivalent to the classical half-space problem of the linearized Boltzmann equation for which the mathematical structure (including the existence and uniqueness of the solution) was conjectured in [24] and relevant theorems have been proved in $[6,13,22]$. In [56, 57], it is explained in detail how to apply the theorem in [6] to the problem (100). According to [56, 57], the problem has a unique solution only when $\breve{T}$ and $\check{v}$ are related to $\partial \hat{T} / \partial x_{j}$ and $\partial \hat{v}_{i} / \partial x_{j}$ appropriately. This relation gives the desired boundary conditions for the compressible Navier-Stokes equations (50), as we will see below.

So far no mention has been made of the initial condition for the Knudsen layer. As mentioned in Sect. 5.1, the Chapman-Enskog solution can be made to satisfy the kinetic initial condition (25) with the choice (51). From the form of (55) and (57), it follows that $\hat{f}_{\mathrm{K}}^{(1)}=0$ or $\phi=0$ should hold at $\tilde{t}=0$. However, since (100a) does not contain the time-derivative term, the initial condition cannot be imposed on $\phi$. In other 
words, the time variable $\tilde{t}$ is just a parameter in the system (100). At $\tilde{t}=0$ (i.e., $\hat{t}=0$ ), because of the uniform initial state (51), $\breve{T}$ and $\breve{v}_{i}$ as well as the terms with subscript B in (100b) vanish. Therefore, the system (100) gives the trivial solution $\phi=0$. In this way, one can see that the requirement imposed on $\phi$ at $\tilde{t}=0$ is fulfilled under assumption (iv) in Sect. 2.

If the initial state does not satisfy assumption (iv), we have to change the initial condition (8) or (25), that is,

$$
f(0, \boldsymbol{X}, \boldsymbol{\xi})=f_{\text {ini }}(\boldsymbol{X}, \boldsymbol{\xi}), \quad \hat{f}(0, \boldsymbol{x}, \boldsymbol{\zeta})=\hat{f}_{\text {ini }}(\boldsymbol{x}, \boldsymbol{\zeta}),
$$

where $f_{\text {ini }}$ is an arbitrary function of $\boldsymbol{X}$ and $\boldsymbol{\xi}$, and $\hat{f}_{\text {ini }}$ is its dimensionless version. In this case, the Chapman-Enskog solution, in general, cannot be made to satisfy this condition, and the solution of the dimensionless Boltzmann equation (15) undergoes a rapid change described by the fast time variable, say $t^{\prime}=\hat{t} / \varepsilon=\overline{v_{c}} t$, in the time interval $0<t^{\prime}<O(1)$ (the so-called initial layer). Moreover, in the vicinity of the boundary, the initial layer interacts with the Knudsen layer. This interaction is an open problem, which is beyond the scope of the present paper. However, with the density $\hat{\rho}_{\text {ini }}$, flow velocity $\hat{v}_{\text {ini }}$, and temperature $\hat{T}_{\text {ini }}$ calculated from $\hat{f}_{\text {ini }}$ with the help of (14a)-(14c), we can set the initial condition

$$
\hat{\rho}=\hat{\rho}_{\text {ini }}(\boldsymbol{x}), \quad \hat{\boldsymbol{v}}=\hat{\boldsymbol{v}}_{\text {ini }}(\boldsymbol{x}), \quad \hat{T}=\hat{T}_{\text {ini }}(\boldsymbol{x}), \quad \text { at } \hat{t}=0,
$$

for the compressible Navier-Stokes equations (50) in place of (51). Then, the system composed of (50), the initial condition (103), and the slip boundary conditions (118) derived later seems to be formally well posed. Of course, this system cannot describe the initial rapid change contained in the solution of the original Boltzmann system, and this initial discrepancy may propagate at later times. However, in the problems in which the flow field is controlled by the slow motion of the boundary or slow variation of the boundary temperature in the time variable $\hat{t}$ that takes place continuously after $\hat{t}=0$, the initial discrepancy may not propagate at later time. Therefore, the solution of the system consisting of (50), (103), and (118) should give the correct approximate solution [with the error of $O\left(\varepsilon^{2}\right)$ ] of the original Boltzmann system except in the initial stage and except in the Knudsen layer.

\subsection{Slip Boundary Conditions}

\subsubsection{Decomposition of the Knudsen-Layer Problem}

If we let the four terms containing the boundary values of the derivatives $\left(\partial \hat{T} / \partial x_{i}\right)_{\mathrm{B}}$ and $\left(\partial \hat{v}_{i} / \partial x_{j}\right)_{\mathrm{B}}$ in (100b) be zero, then the problem (100) has a trivial solution $\phi=0$, $\breve{T}=0$, and $\check{v}_{i}=0$, which is unique according to [6]. In this sense, it is a homogeneous problem. When the boundary condition at $y=0$ has inhomogeneous terms as in the case of (100b), the solution $\phi$ as well as $\check{T}$ and $\check{v}_{i}$ is determined depending on the inhomogeneous terms. Since the problem is linear, we can decompose the problem (100) in accordance with the form of the inhomogeneous terms.

Let us denote by $\boldsymbol{t}$ and $\boldsymbol{s}$ two unit vectors on the plane tangent to the boundary, which are orthogonal to each other and fixed to the boundary, i.e., $\boldsymbol{n} \cdot \boldsymbol{t}=0, \boldsymbol{n} \cdot \boldsymbol{s}=0$, 
and $\boldsymbol{t} \cdot \boldsymbol{s}=0$. Then, $\delta_{i j}-n_{i} n_{j}=t_{i} t_{j}+s_{i} s_{j}$ holds. From the form of the inhomogeneous terms in (100b), we seek the solution in the following form:

$$
\begin{aligned}
\phi\left(\tilde{t}, y, \chi_{1}, \chi_{2}, \zeta\right)= & \frac{1}{\hat{\rho}_{\mathrm{B}} \hat{T}_{\mathrm{w}}^{1 / 2}}\left[\left(\frac{\partial \hat{v}_{j}}{\partial x_{i}}\right)_{\mathrm{B}}+\left(\frac{\partial \hat{v}_{i}}{\partial x_{j}}\right)_{\mathrm{B}}\right] \zeta_{l} n_{i}\left(\delta_{j l}-n_{j} n_{l}\right) \phi_{v}^{I}\left(y, \zeta_{\mathrm{n}}, \zeta\right) \\
& +\frac{1}{\hat{\rho}_{\mathrm{B}} \hat{T}_{\mathrm{w}}}\left(\frac{\partial \hat{T}}{\partial x_{j}}\right)_{\mathrm{B}} \zeta_{i}\left(\delta_{i j}-n_{i} n_{j}\right) \phi_{T}^{I}\left(y, \zeta_{\mathrm{n}}, \zeta\right) \\
& +\frac{1}{\hat{\rho}_{\mathrm{B}} \hat{T}_{\mathrm{w}}^{1 / 2}}\left(\frac{\partial \hat{v}_{i}}{\partial x_{j}}\right)_{\mathrm{B}} n_{i} n_{j} \phi_{v}^{I I}\left(y, \zeta_{\mathrm{n}}, \zeta\right) \\
& +\frac{1}{\hat{\rho}_{\mathrm{B}} \hat{T}_{\mathrm{w}}}\left(\frac{\partial \hat{T}}{\partial x_{i}}\right)_{\mathrm{B}} n_{i} \phi_{T}^{I I}\left(y, \zeta_{\mathrm{n}}, \zeta\right),
\end{aligned}
$$

and

$$
\begin{aligned}
& \frac{\check{v}_{i}}{\hat{T}_{\mathrm{W}}^{1 / 2}} t_{i}=c_{v}^{I} \frac{1}{\hat{\rho}_{\mathrm{B}} \hat{T}_{\mathrm{W}}^{1 / 2}}\left[\left(\frac{\partial \hat{v}_{i}}{\partial x_{j}}\right)_{\mathrm{B}}+\left(\frac{\partial \hat{v}_{j}}{\partial x_{i}}\right)_{\mathrm{B}}\right] n_{i} t_{j}+c_{T}^{I} \frac{1}{\hat{\rho}_{\mathrm{B}} \hat{T}_{\mathrm{W}}}\left(\frac{\partial \hat{T}}{\partial x_{i}}\right)_{\mathrm{B}} t_{i} \\
& \frac{\check{T}}{\hat{T}_{\mathrm{W}}}=c_{v}^{I I} \frac{1}{\hat{\rho}_{\mathrm{B}} \hat{T}_{\mathrm{w}}^{1 / 2}}\left(\frac{\partial \hat{v}_{i}}{\partial x_{j}}\right)_{\mathrm{B}} n_{i} n_{j}+c_{T}^{I I} \frac{1}{\hat{\rho}_{\mathrm{B}} \hat{T}_{\mathrm{w}}}\left(\frac{\partial \hat{T}}{\partial x_{i}}\right)_{\mathrm{B}} n_{i}
\end{aligned}
$$

where $c_{v}^{I}, c_{T}^{I}, c_{v}^{I I}$, and $c_{T}^{I I}$ are undetermined quantities, which are determined together with the solutions $\phi_{v}^{I}, \phi_{T}^{I}, \phi_{v}^{I I}$, and $\phi_{T}^{I I}$. Since the direction of $\boldsymbol{t}$ is arbitrary, we do not need the expression for $\boldsymbol{s}$ corresponding to (105a). As we will see below, $c_{v}^{I}, c_{T}^{I}, c_{v}^{I I}$, and $c_{T}^{I I}$ as well as $\phi_{v}^{I}, \phi_{T}^{I}, \phi_{v}^{I I}$, and $\phi_{T}^{I I}$, in general, depend on $\hat{T}_{\mathrm{w}}$. The assumption that $\phi_{v}^{I}, \phi_{T}^{I}, \phi_{v}^{I I}$, and $\phi_{T}^{I I}$ are all functions of $y, \zeta_{\mathrm{n}}$, and $\zeta$ only will turn out to be consistent.

The substitution of (104) and (105) into (100) leads to the four problems for $\phi_{v}^{I}, \phi_{T}^{I}$, $\phi_{v}^{I I}$, and $\phi_{T}^{I I}$. That is,

- Problem for $\phi_{v}^{I}$ :

$$
\begin{aligned}
& \zeta_{\mathrm{n}} \frac{\partial \phi_{v}^{I}}{\partial y}=\mathscr{L}_{\hat{T}_{\mathrm{w}}}^{S}\left(\phi_{v}^{I}\right), \quad(y>0), \\
& \phi_{v}^{I}=(1-\alpha) \widetilde{\mathrm{R}} \phi_{v}^{I}-2 \alpha c_{v}^{I}+(2-\alpha) \zeta_{\mathrm{n}} \mathscr{B}^{(0)}\left(\zeta, \hat{T}_{\mathrm{w}}\right), \quad\left(y=0, \zeta_{\mathrm{n}}>0\right) \\
& \phi_{v}^{I} \rightarrow 0, \quad(y \rightarrow \infty) .
\end{aligned}
$$

- Problem for $\phi_{T}^{I}$ :

$$
\begin{aligned}
& \zeta_{\mathrm{n}} \frac{\partial \phi_{T}^{I}}{\partial y}=\mathscr{L}_{\hat{T}_{\mathrm{w}}}^{S}\left(\phi_{T}^{I}\right), \quad(y>0), \\
& \phi_{T}^{I}=(1-\alpha) \widetilde{\mathrm{R}} \phi_{T}^{I}-2 \alpha c_{T}^{I}+\alpha \mathscr{A}\left(\zeta, \hat{T}_{\mathrm{w}}\right), \quad\left(y=0, \zeta_{\mathrm{n}}>0\right), \\
& \phi_{T}^{I} \rightarrow 0, \quad(y \rightarrow \infty) .
\end{aligned}
$$


- Problem for $\phi_{v}^{I I}$ :

$$
\begin{aligned}
& \zeta_{\mathrm{n}} \frac{\partial \phi_{v}^{I I}}{\partial y}=\mathscr{L}_{\hat{T}_{\mathrm{w}}}\left(\phi_{v}^{I I}\right), \quad(y>0), \\
& \phi_{v}^{I I}=(1-\alpha) \widetilde{\mathrm{R}} \phi_{v}^{I I}-2 \alpha \sqrt{\pi} \int_{\zeta_{\mathrm{n}}<0} \zeta_{\mathrm{n}} \phi_{v}^{I I} E(\zeta) d \zeta-\alpha\left(\zeta^{2}-2\right) c_{v}^{I I} \\
& \quad+\alpha\left[\left(\zeta_{\mathrm{n}}^{2}-\frac{1}{3} \zeta^{2}\right) \mathscr{B}^{(0)}\left(\zeta, \hat{T}_{\mathrm{w}}\right)-\frac{1}{3} \mathscr{I}_{\mathscr{B}}\right], \quad\left(y=0, \zeta_{\mathrm{n}}>0\right), \\
& \phi_{v}^{I I} \rightarrow 0, \quad(y \rightarrow \infty) .
\end{aligned}
$$

- Problem for $\phi_{T}^{I I}$ :

$$
\begin{aligned}
& \begin{array}{l}
\zeta_{\mathrm{n}} \frac{\partial \phi_{T}^{I I}}{\partial y}=\mathscr{L}_{\hat{T}_{\mathrm{w}}}\left(\phi_{T}^{I I}\right), \quad(y>0), \\
\phi_{T}^{I I}=(1-\alpha) \widetilde{\mathrm{R}} \phi_{T}^{I I}-2 \alpha \sqrt{\pi} \int_{\zeta_{\mathrm{n}}<0} \zeta_{\mathrm{n}} \phi_{T}^{I I} E(\zeta) d \zeta-\alpha\left(\zeta^{2}-2\right) c_{T}^{I I} \\
\quad+(2-\alpha) \zeta_{\mathrm{n}} \mathscr{A}\left(\zeta, \hat{T}_{\mathrm{w}}\right), \quad\left(y=0, \zeta_{\mathrm{n}}>0\right),
\end{array} \\
& \phi_{T}^{I I} \rightarrow 0, \quad(y \rightarrow \infty) .
\end{aligned}
$$

In (106a) and (107a), $\mathscr{L}_{\hat{T}_{\mathrm{w}}}^{S}(\cdot)$ is a linear operator defined as follows: It is well known [57] that for any function $\varphi\left(\zeta_{\mathrm{n}}, \zeta\right)$ of $\zeta_{\mathrm{n}}$ and $\zeta$, the relation $\mathscr{L}_{\hat{T}_{\mathrm{w}}}\left[\zeta_{j} t_{j} \varphi\left(\zeta_{\mathrm{n}}, \zeta\right)\right]=\zeta_{j} t_{j} \times$ (a function of $\zeta_{\mathrm{n}}$ and $\zeta$ ) holds; $\mathscr{L}_{\hat{T}_{\mathrm{w}}}^{S}(\cdot)$ is defined by this resulting function of $\zeta_{\mathrm{n}}$ and $\zeta$, namely,

$$
\mathscr{L}_{\hat{T}_{\mathrm{w}}}\left[\zeta_{j} t_{j} \varphi\left(\zeta_{\mathrm{n}}, \zeta\right)\right]=\zeta_{j} t_{j} \mathscr{L}_{\hat{T}_{\mathrm{w}}}^{S}\left[\varphi\left(\zeta_{\mathrm{n}}, \zeta\right)\right]\left(\zeta_{\mathrm{n}}, \zeta\right)
$$

where the last parentheses show the independent variables of $\mathscr{L}_{\hat{T}_{\mathrm{w}}}^{S}(\varphi)$.

For the BGK model, the right-hand side of (106a) and that of (107a) are replaced with $\mathscr{L}_{\mathrm{BGK}}^{S}\left(\phi_{v}^{I}\right)$ and $\mathscr{L}_{\mathrm{BGK}}^{S}\left(\phi_{T}^{I}\right)$, respectively, and the right-hand side of (108a) and that of (109a) are replaced with $\mathscr{L}_{\mathrm{BGK}}\left(\phi_{v}^{I I}\right)$ and $\mathscr{L}_{\mathrm{BGK}}\left(\phi_{T}^{I I}\right)$, respectively. Here, $\mathscr{L}_{\mathrm{BGK}}^{S}(\cdot)$ is defined in the same way as (110), that is, it is given by

$$
\mathscr{L}_{\mathrm{BGK}}^{S}\left[\varphi\left(\zeta_{\mathrm{n}}, \zeta\right)\right]=\int\left(\zeta_{*}^{2}-\zeta_{* \mathrm{n}}^{2}\right) \varphi\left(\zeta_{* \mathrm{n}}, \zeta_{*}\right) E\left(\zeta_{*}\right) d \zeta_{*}-\varphi\left(\zeta_{\mathrm{n}}, \zeta\right)
$$

\subsubsection{Solutions to Decomposed Half-Space Problems}

Since $\mathscr{L}_{\hat{T}_{\mathrm{w}}}, \mathscr{L}_{\hat{T}_{\mathrm{w}}}^{S}, \mathscr{A}$, and $\mathscr{B}^{(0)}$ generally depend on $\hat{T}_{\mathrm{w}}$, the solutions $\phi_{v}^{I}, \phi_{T}^{I}, \phi_{v}^{I I}$, and $\phi_{T}^{I I}$ as well as the undetermined quantities $c_{v}^{I}, c_{T}^{I}, c_{v}^{I I}$, and $c_{T}^{I I}$, in general, depend on $\hat{T}_{\mathrm{w}}$. As we have remarked in Sects. 4.1 and 4.2, for hard-sphere molecules, $\mathscr{L}_{\hat{T}_{\mathrm{w}}}, \mathscr{A}$, and $\mathscr{B}^{(0)}$ are independent of $\hat{T}_{\mathrm{w}}$, i.e.,

$$
\mathscr{L}_{\hat{T}_{\mathrm{w}}}(\cdot)=\mathscr{L}(\cdot), \quad \mathscr{A}\left(\zeta, \hat{T}_{\mathrm{w}}\right)=A(\zeta), \quad \mathscr{B}^{(0)}\left(\zeta, \hat{T}_{\mathrm{w}}\right)=B(\zeta),
$$


hold, so that $\mathscr{L}_{\hat{T}_{\mathrm{w}}}^{S}(\cdot)$ is also independent of $\hat{T}_{\mathrm{w}}$ and may be denoted by $\mathscr{L}^{S}(\cdot)$. Therefore, the solutions $\phi_{v}^{I}, \phi_{T}^{I}, \phi_{v}^{I I}$, and $\phi_{T}^{I I}$ as well as the undetermined quantities $c_{v}^{I}, c_{T}^{I}, c_{v}^{I I}$, and $c_{T}^{I I}$ are independent of $\hat{T}_{\mathrm{w}}$. On the other hand, for the BGK model, though $\mathscr{L}_{\mathrm{BGK}}$ and $\mathscr{L}_{\mathrm{BGK}}^{S}$ are independent of $\hat{T}_{\mathrm{w}}, \mathscr{A}$ and $\mathscr{B}^{(0)}$ depend on it. More specifically, from (38) and (89b), we have

$$
\mathscr{A}\left(\zeta, \hat{T}_{\mathrm{w}}\right)=\frac{\hat{T}_{\mathrm{w}}^{1 / 2}}{\hat{A}_{c}\left(\hat{T}_{\mathrm{w}}\right)}\left(\zeta^{2}-\frac{5}{2}\right), \quad \mathscr{B}^{(0)}\left(\zeta, \hat{T}_{\mathrm{w}}\right)=\frac{2 \hat{T}_{\mathrm{w}}^{1 / 2}}{\hat{A}_{c}\left(\hat{T}_{\mathrm{w}}\right)}, \quad \mathscr{I}_{\mathscr{B}}=\frac{2 \hat{T}_{\mathrm{w}}^{1 / 2}}{\hat{A}_{c}\left(\hat{T}_{\mathrm{w}}\right)} .
$$

In the following, we discuss each of these problems, putting the problem for $\phi_{v}^{I I}$ at the end.

\section{- Problem for $\phi_{v}^{I}$}

If $\hat{T}_{\mathrm{w}}=1$, the problem for $\phi_{v}^{I},(106)$, is the same as the classical shear-slip problem (the so-called Kramers problem; see, e.g., [12, 50, 36, 32]). Let us first restrict ourselves to the case of diffuse reflection $(\alpha=1)$. For hard-sphere molecules, the latter problem was solved numerically in [36]. Since the problem (106) does not depend on $\hat{T}_{\mathrm{w}}$ for hard-sphere molecules, its solution is immediately given by the result in [36], that is, $\left(y, \zeta_{i} n_{i}, \zeta_{i} t_{i}, \zeta_{i} s_{i}, \phi_{v}^{I}, c_{v}^{I}\right)$ (here) $=$ $\left[x_{1}, \zeta_{1}, \zeta_{2}, \zeta_{3},\left(\zeta_{2}^{2}+\zeta_{3}^{2}\right)^{-1 / 2} \Phi_{A}, \beta_{A}\right]$ (in [36]) and $c_{v}^{I}$ (here) $=-k_{0}$ (in [57]; see Sect. 3.1.5 in [57]). It should be noted that a more accurate value of $c_{v}^{I}$ has been obtained in a recent paper [25]: $c_{v}^{I}$ (here) $=b_{1}^{(1)}$ (in [25]; see also the supplementary note on the Kyoto University Research Information Repository, http://hdl.handle.net/2433/199811). For the BGK model, an accurate numerical solution for the shear-slip problem is contained in [64]. Because of the form (113), if we let $\left(\phi_{v}^{I}, c_{v}^{I}\right)=\left[\hat{T}_{\mathrm{w}}^{1 / 2} / \hat{A}_{c}\left(\hat{T}_{\mathrm{w}}\right)\right]\left(\hat{\phi}_{v}^{I}, \hat{c}_{v}^{I}\right)$, then $\left(\hat{\phi}_{v}^{I}, \hat{c}_{v}^{I}\right)$ are given by the latter reference. More specifically, we have $c_{v}^{I}$ (here) $=\left[\hat{T}_{\mathrm{w}}^{1 / 2} / \hat{A}_{c}\left(\hat{T}_{\mathrm{w}}\right)\right] \times\left[-k_{0}\right.$ (in $[64,57])]$. In summary, we have the following result for the diffuse reflection:

$$
c_{v}^{I}=1.25395 \quad \text { (hard sphere) [25], } \quad c_{v}^{I}=1.01619 \frac{\hat{T}_{\mathrm{w}}^{1 / 2}}{\hat{A}_{c}\left(\hat{T}_{\mathrm{w}}\right)} \text { (BGK) [57] }
$$

The shear-slip problem has also been solved numerically for the Maxwell-type condition with $0<\alpha \leq 1$. The reader is referred to, e.g., [33,67, 45] for hardsphere molecules and [38] for the BGK model (see also [44]).

\section{- Problem for $\phi_{T}^{I}$}

When $\hat{T}_{\mathrm{W}}=1$, the problem for $\phi_{T}^{I},(107)$, is the same as the classical thermalcreep problem (see, e.g., [52, 36, 30]). We consider the case of diffuse reflection $(\alpha=1)$. For hard-sphere molecules, the problem was solved numerically in [36]. Since the problem (107) does not depend on $\hat{T}_{\mathrm{w}}$ in this case, its solution is immediately given by the result in [36], that is, $\left(y, \zeta_{i} n_{i}, \zeta_{i} t_{i}, \zeta_{i} s_{i}, \phi_{T}^{I}, c_{T}^{I}\right)$ (here) $=$ 
$\left[x_{1}, \zeta_{1}, \zeta_{2}, \zeta_{3},\left(\zeta_{2}^{2}+\zeta_{3}^{2}\right)^{-1 / 2} \Phi_{B}, \beta_{B}\right]$ (in [36]) and $c_{T}^{I}$ (here) $=-K_{1}$ (in [57]; see Sect. 3.1.5 in [57]). We note that a more accurate value of $c_{T}^{I}$ has been obtained in [25]: $c_{T}^{I}$ (here) $=b_{2}^{(1)}$ (in [25]). For the BGK model, an accurate numerical solution for the thermal-creep problem is contained in [64], and for the same reason as in the problem for $\phi_{v}^{I}$, if we let $\left(\phi_{T}^{I}, c_{T}^{I}\right)=\left[\hat{T}_{\mathrm{w}}^{1 / 2} / \hat{A}_{c}\left(\hat{T}_{\mathrm{w}}\right)\right]\left(\hat{\phi}_{T}^{I}, \hat{c}_{T}^{I}\right)$, then $\left(\hat{\phi}_{T}^{I}, \hat{c}_{T}^{I}\right)$ are given by the result in [64]. More specifically, $c_{T}^{I}$ (here) $=$ $\left[\hat{T}_{\mathrm{w}}^{1 / 2} / \hat{A}_{c}\left(\hat{T}_{\mathrm{w}}\right)\right] \times\left[-K_{1}\right.$ (in $\left.\left.[64,57]\right)\right]$. In summary, we have the following result for the diffuse reflection:

$$
c_{T}^{I}=0.64642 \text { (hard sphere) [25], } \quad c_{T}^{I}=0.38316 \frac{\hat{T}_{\mathrm{w}}^{1 / 2}}{\hat{A}_{c}\left(\hat{T}_{\mathrm{w}}\right)} \quad \text { (BGK) [57]. }
$$

The thermal-creep problem has also been solved numerically for the Maxwelltype condition with $0<\alpha \leq 1$. The reader is referred to, e.g., [45] for hardsphere molecules and [37] for the BGK model (see also [44]).

- Problem for $\phi_{T}^{I I}$

If $\hat{T}_{\mathrm{w}}=1$, the problem for $\phi_{T}^{I I},(109)$, is identical to the classical temperaturejump problem (see, e.g., [68, 51, 31, 63, 49, 62, 30]). We consider the case of diffuse reflection $(\alpha=1)$. For hard-sphere molecules, the problem was solved numerically in [62] (see also [35]). Since the problem (109) does not depend on $\hat{T}_{\mathrm{w}}$ in this case, its solution is immediately obtained from the result in [62], that is, $\left(y, \zeta_{i} n_{i}, \zeta_{i} t_{i}, \zeta_{i} s_{i}, \phi_{T}^{I I}, c_{T}^{I I}\right)$ (here) $=\left(x_{1}, \zeta_{1}, \zeta_{2}, \zeta_{3}, \Phi_{\mathrm{K}}, \beta\right)$ (in [62]) $=\left(\eta, \zeta_{1}, \zeta_{2}, \zeta_{3}, \Phi_{1}, b\right)$ (in [35]) and $c_{T}^{I I}$ (here) $=d_{1}$ (in [57]; see Sect. 3.1 .5 in [57]). We should note that a more accurate value of $c_{T}^{I I}$ has been obtained in [25]: $c_{T}^{I I}$ (here) $=c_{1}^{(0)}$ (in [25]). As for the BGK model, an accurate numerical solution for the temperaturejump problem is contained in $[63,64]$. As in the problems for $\phi_{v}^{I}$ and $\phi_{T}^{I}$, if we let $\left(\phi_{T}^{I I}, c_{T}^{I I}\right)=\left[\hat{T}_{\mathrm{w}}^{1 / 2} / \hat{A}_{c}\left(\hat{T}_{\mathrm{w}}\right)\right]\left(\hat{\phi}_{T}^{I I}, \hat{c}_{T}^{I I}\right)$, then $\left(\hat{\phi}_{T}^{I I}, \hat{c}_{T}^{I I}\right)$ are given by the latter references. Concerning the coefficient $c_{T}^{I I}$, therefore, we have $c_{T}^{I I}$ (here) $=$ $\left[\hat{T}_{\mathrm{w}}^{1 / 2} / \hat{A}_{c}\left(\hat{T}_{\mathrm{w}}\right)\right] \times\left[d_{1}\right.$ (in $\left.\left.[63,64,57]\right)\right]$. In summary, we have the following result for the diffuse reflection:

$$
c_{T}^{I I}=2.40014 \quad \text { (hard sphere) [25], } \quad c_{T}^{I I}=1.30272 \frac{\hat{T}_{\mathrm{w}}^{1 / 2}}{\hat{A}_{c}\left(\hat{T}_{\mathrm{w}}\right)} \quad \text { (BGK) [57]. }
$$

The temperature-jump problem has also been solved numerically for the Maxwelltype condition with $0<\alpha \leq 1$. The reader is referred to, e.g., [46] for hardsphere molecules and [39] for the BGK model (see also [44]).

\section{- Problem for $\phi_{v}^{I I}$}

Unlike the other three problems, the problem for $\phi_{v}^{I I},(108)$, has been studied rarely because it does not correspond to a specific half-space problem of physical 
interest. This problem with $\hat{T}_{\mathrm{w}}=1$ has appeared only in the generalized slip flow theory (linear theory) with evaporation and condensation on the boundary [64, 57] (BGK model) and that with solid boundary for time-dependent problems [65, $25,26]$ (hard-sphere molecules). The comparison of (108) with these references gives the following relationship: $\left(y, \zeta_{\mathrm{n}}, \zeta, \phi_{v}^{I I}, c_{v}^{I I}\right)$ (here) $=\left(\eta, \mu \zeta, \zeta, \phi_{5}, c_{5}^{(0)}\right)$ (in [25]) for hard-sphere molecules, and $c_{v}^{I I}$ (here) $=(4 / 3)\left[\hat{T}_{\mathrm{w}}^{1 / 2} / \hat{A}_{c}\left(\hat{T}_{\mathrm{w}}\right)\right] \times\left[d_{6}\right.$ (in $[64,57])]$ for the BGK model. To summarize, we have the following result of $c_{v}^{I I}$ for the diffuse reflection:

$$
c_{v}^{I I}=0.45957 \quad \text { (hard sphere) [25], } \quad c_{v}^{I I}=0.44045 \frac{\hat{T}_{\mathrm{w}}^{1 / 2}}{\hat{A}_{c}\left(\hat{T}_{\mathrm{w}}\right)} \quad(\mathrm{BGK})[57]
$$

\subsubsection{Summary of the Slip Boundary Conditions}

From (59), (95), and (105), we can immediately write down the slip boundary conditions for the compressible Navier-Stokes equations, (50) with (47), in the following form:

$$
\begin{aligned}
& \left(\hat{v}_{i}-\hat{v}_{\mathrm{w} i}\right) n_{i}=0 \\
& \left(\hat{v}_{i}-\hat{v}_{\mathrm{w} i}\right) t_{i}=\varepsilon c_{v}^{I} \frac{1}{\hat{\rho}}\left(\frac{\partial \hat{v}_{i}}{\partial x_{j}}+\frac{\partial \hat{v}_{j}}{\partial x_{i}}\right) n_{i} t_{j}+\varepsilon c_{T}^{I} \frac{1}{\hat{\rho} \hat{T}_{\mathrm{w}}^{1 / 2}} \frac{\partial \hat{T}}{\partial x_{i}} t_{i} \\
& \hat{T}-\hat{T}_{\mathrm{w}}=\varepsilon c_{v}^{I I} \frac{\hat{T}_{\mathrm{w}}^{1 / 2}}{\hat{\rho}} \frac{\partial \hat{v}_{i}}{\partial x_{j}} n_{i} n_{j}+\varepsilon c_{T}^{I I} \frac{1}{\hat{\rho}} \frac{\partial \hat{T}}{\partial x_{i}} n_{i},
\end{aligned}
$$

where the quantities $\hat{\rho}, \hat{v}_{i}$, and $\hat{T}$, which belong to the Chapman-Enskog solution, as well as their derivatives are all evaluated on the boundary. Recall that the so-called slip coefficients $c_{v}^{I}, c_{T}^{I}, c_{v}^{I I}$, and $c_{T}^{I I}$, in general, depend on $\hat{T}_{\mathrm{w}}$. Their explicit expressions for the hard-sphere molecules as well as for the BGK model under the diffuse-reflection condition are summarized in (114), (115), (117), and (116).

At a glance, the slip boundary conditions (118) seem to be the same as the ones derived in Sone's generalized slip flow theory (linear theory), e.g., the uppermost lines of (3.60a)-(3.60c) in [56]. However, there are important differences. Unlike (3.60) in [56], the slip conditions (118) are nonlinear because of the factor $1 / \hat{\rho}$, which does not occur in (3.60) in [56], and the slip coefficients $c_{v}^{I}, c_{T}^{I}, c_{v}^{I I}$, and $c_{T}^{I I}$ are in general not

constant but dependent on $\hat{T}_{\mathrm{w}}$. In addition, the term containing $c_{v}^{I I}$ does not appear in (3.60) in [56] because it becomes higher order in $\varepsilon$ for flows with small Mach numbers. For the compressible Navier-Stokes equations, these effects are important and should be taken into account precisely. The relation between (118) and the slip boundary conditions appearing in Sone's generalized slip flow theory, including (3.60) in [56], will be discussed in Sect. 5.3.4.

From the process of its derivation, one might think that the slip boundary condition (118) should be applied to points fixed on the boundary, following their trajectories. However, in the Knudsen-layer problem (100), the time variable $\tilde{t}$ (or $\hat{t}$ ) is contained 
only as a parameter, that is, the time-derivative term is not contained. Therefore, the solution $\phi$ as well as the resulting slip-boundary conditions do not depend on the trajectory of the boundary point $\boldsymbol{x}_{\mathrm{w}}\left(\hat{t}, \chi_{1}, \chi_{2}\right)$ in the past. In other words, we do not need to follow the trajectories of the boundary points. The conditions (118) form twodimensional fields on the boundary at each time. For example, when we consider a time-independent problem in which the boundary is moving in its surface (e.g., cylindrical Couette flow between two coaxial circular cylinders rotating at different angular speeds), we can impose the conditions (118) at each point on the boundary that is fixed in the coordinate system, not at the point moving with the boundary, as is usually done.

Incidentally, the initial condition for (50) is given by (51) or (103) (see the discussion in the last two paragraphs of Sect. 5.2.4).

As we have seen in Sect. 5.1, if we assume the no-slip condition (52), the firstorder Chapman-Enskog solution $\hat{f}^{(0)}+\hat{f}^{(1)} \varepsilon$ satisfies the kinetic boundary condition (27) only at the zeroth-order in $\varepsilon$. This is not consistent with the first-order ChapmanEnskog solution, in which the terms of $O(\varepsilon)$ are taken into account. In other words, the no-slip boundary condition is not consistent with the compressible Navier-Stokes equations that correspond to the first-order Chapman-Enskog solution. In order to be consistent, we have to combine the slip boundary conditions (118), not the no-slip condition (52), with the compressible Navier-Stokes equations (50) for any small $\varepsilon$.

As shown in Sects. 5.3.1 and 5.3.2, the problem of deriving the slip boundary conditions in the fully nonlinear setting is reduced to the fundamental half-space problems of the linearized Boltzmann equation. In particular, the problems of obtaining $c_{v}^{I}, c_{T}^{I}$, and $c_{T}^{I I}$ are the classical problems, and nowadays their numerical solutions under other types of kinetic boundary conditions, such as the Cercignani-Lampis condition [15], are also available (e.g., [48, 43, 47, 21]). Since the same reduction should hold for the Cercignani-Lampis condition, one can in principle extend (118) to this condition by exploiting the available results for the problems in which the term containing $c_{v}^{I I}$ becomes higher order in $\varepsilon$, e.g., Taylor-Couette problem [2].

\subsubsection{Additional Remarks on the Slip Boundary Conditions}

First, we briefly discuss the relation of the slip boundary conditions (118) with those appearing in Sone's generalized slip flow theory $[56,57]$. For clearness, we restrict ourselves to steady problems and refer to [56] for comparison below. In the ChapmanEnskog expansion, on which our analysis is based, the macroscopic quantities are not expanded in $\varepsilon$, whereas in the analysis in [56, 57], in which Hilbelt-type expansions are used, they are expansed in $\varepsilon$. Therefore, in order to compare the results, we need to re-expand the macroscopic quantities. However, the appropriate re-expansion depends on the physical situation, so that we make the comparison separately below.

Let us start with the case where the deviation from the reference equilibrium state at rest is small. Then, we can express $\hat{v}_{\mathrm{w} i}$ and $\hat{T}_{\mathrm{w}}$ as $\hat{v}_{\mathrm{w} i}=0+u_{\mathrm{w} i}$ and $\hat{T}_{\mathrm{w}}=1+\tau_{\mathrm{w}}$ with small deviations $u_{\mathrm{w} i}$ and $\tau_{\mathrm{w}}$. Accordingly, $\hat{\rho}, \hat{v}_{i}, \hat{T}$, and $\hat{p}$ can be expressed as $\hat{\rho}=1+\omega, \hat{v}_{i}=0+u_{i}, \hat{T}=1+\tau$, and $\hat{p}=1+P$ with small deviations $\omega, u_{i}, \tau$, and $P$, which will be represented by $\tilde{h}$ below $\left(\tilde{h}=\omega, u_{i}, \tau\right.$, and $\left.P\right)$.

(i) Linear problems: When the deviations $u_{\mathrm{w} i}, \tau_{\mathrm{w}}$, and thus $\tilde{h}$ are much smaller than 
$\varepsilon$ (we assume that $u_{\mathrm{w} i}$ and $\tau_{\mathrm{w}}$ are independent of $\varepsilon$ ), we can first linearize the Navier-Stokes equations (50) and the slip boundary conditions (118) by neglecting the product terms of the deviations. Then, we expand $\tilde{h}$ as $\tilde{h}=\tilde{h}_{0}+\tilde{h}_{1} \varepsilon+\cdots$ (note that $\tilde{h}_{0}$ and $\tilde{h}_{1}$ are much smaller than $\varepsilon$ ). If we insert these expansions in the linearized Navier-Stokes equations, we obtain (3.18) and (3.19) (with $m=0$ and 1) in [56], which are the Stokes system of equations (note that $\varepsilon$ here is the same as $k$ in [56] and ignore the subscript $G$ in the result of [56]). If we use the expansions in the linearized slip boundary conditions, we obtain (3.59a) with $u_{w i 0}=u_{\mathrm{w} i}$ and (3.59b) with $\tau_{w 0}=\tau_{\mathrm{w}}$ (the no-slip conditions) and the uppermost lines of (3.60a)-(3.60c) with $u_{w i 1}=\tau_{w 1}=0$ (the linearized slip boundary conditions) in [56]. Here, one should note that the term $\left(\partial u_{i 0} / \partial x_{j}\right) n_{i} n_{j}$, originating from the term $\left(\hat{T}_{\mathrm{w}}^{1 / 2} / \hat{\rho}\right)\left(\partial \hat{v}_{i} / \partial x_{j}\right) n_{i} n_{j}$ in (118c), vanishes because of the relation $n_{i} n_{j}=\delta_{i j}-\left(t_{i} t_{j}+s_{i} s_{j}\right)$, the continuity equations (3.19a) (with $m=0$ ) in [56], and the fact that $\left(\partial u_{i 0} / \partial x_{j}\right)\left(t_{i} t_{j}+s_{i} s_{j}\right)=\left(\partial u_{\mathrm{wi} 0} / \partial x_{j}\right)\left(t_{i} t_{j}+s_{i} s_{j}\right)=$ $(1 / 2)\left(\partial u_{\mathrm{w} i 0} / \partial x_{j}+\partial u_{\mathrm{w} j 0} / \partial x_{i}\right)\left(t_{i} t_{j}+s_{i} s_{j}\right)=0$ (rigid-body motion). In this way, the Stokes set of equations and its first-order slip boundary conditions of the linear theory of [56] is recovered from (50) and (118) by the linearization and an appropriate re-expansion.

(ii) Weakly nonlinear problems: When the deviations $u_{\mathrm{w} i}, \tau_{\mathrm{w}}$, and thus $\tilde{h}$ are of the order of $\varepsilon$, we should set $u_{\mathrm{w} i}=u_{\mathrm{w} i 1} \varepsilon$ and $\tau_{\mathrm{w}}=\tau_{\mathrm{w} 1} \varepsilon$ (we assume that $u_{\mathrm{w} i 1}$ and $\tau_{\mathrm{w} 1}$ are independent of $\varepsilon$ ) and expand $\tilde{h}$ as $\tilde{h}=\tilde{h}_{1} \varepsilon+\tilde{h}_{2} \varepsilon^{2}+\cdots$ [note that the expansions start from $O(\varepsilon)$ and that $\tilde{h}_{1}$ and $\tilde{h}_{2}$ are of $\left.O(1)\right]$. If we substitute these expansions into the Navier-Stokes equations (50), we obtain (4.30), (4.31), (4.32a), (4.32c), and (4.32b) with $\gamma_{3}=0$ in [56] (ignore the subscript $S$ in the result of [56]). Equations (4.31a) and (4.31b) are the so-called incompressible Navier-Stokes equations. On the other hand, the slip boundary conditions (118) with the above expansions give (4.61a) and (4.61b) (the no-slip conditions) and the uppermost lines of (4.62a)-(4.62c) with $u_{w i 2}=\tau_{w 2}=0$ (the slip boundary conditions) in [56]. The term originating from the $c_{v}^{I I}$ term in (118c) becomes of higher order in $\varepsilon$ for the same reason as in (i). In this way, the weakly nonlinear theory of [56] is recovered from (50) and (118) by a suitable expansion except the fact that the term containing $\gamma_{3}$ in (4.32b) in [56] does not appear. This term, which originates from the thermal stress, only modifies the $\varepsilon^{3}$-order pressure field $P_{3}\left(P_{S 3}\right.$ in [56]).

Next we consider fully nonlinear problems in which $\hat{v}_{\mathrm{w} i}, \hat{T}_{\mathrm{w}}, \hat{\rho}, \hat{v}_{i}, \hat{T}$, and $\hat{p}$ are all of $O(1)$.

(iii) Fully nonlinear problems: Let us consider the situation in which the viscous boundary layer appears on the whole boundary. According to [61, 56, 57], the appropriate expansion parameter is $\sqrt{\varepsilon}$. Therefore, we expand $\hat{h}$ as $\hat{h}=$ $\hat{h}_{0}+\hat{h}_{1} \sqrt{\varepsilon}+\cdots$, where $\hat{h}$ stands for $\hat{\rho}, \hat{v}_{i}, \hat{T}$, and $\hat{p}$ (cf. Sect. 5.1) [note that $\varepsilon($ here $)=k([56])$ and $\sqrt{\varepsilon}$ (here) $=\varepsilon([56])]$. If we substitute these expansions into (50), we obtain (6.21)-(6.26) in [56] (ignore the subscript $h$ in the result of [56]), which are the (compressible) Euler set of equations re-expanded in $\sqrt{\varepsilon}$. 
The fact that the expansion parameter should be $\sqrt{\varepsilon}$ is closely related to the appearance of the viscous boundary layer whose thickness (in the dimensionless $x$ space) is of the order of $\sqrt{\varepsilon}$. Once the viscous boundary layer with thickness of the order of $\sqrt{\varepsilon}$ appears, we have to introduce a normal coordinate, say $z$, with the length scale of $\sqrt{\varepsilon}$, i.e., $x_{i}=\sqrt{\varepsilon} z n_{i}+x_{\mathrm{w} i}$ in place of (64b), in order to describe the behavior inside the layer. Then, similar to (71) and (72), we have $\partial / \partial x_{i}=(1 / \sqrt{\varepsilon}) n_{i} \partial / \partial z+O(1)$. With the re-expansion of (50), we should obtain (6.52), (6.53), (6.58), and (6.60) with (6.57) for $\hat{\rho}_{0}, \hat{v}_{i 0}, \hat{T}_{0}$, and $\hat{p}_{0}$ and (6.57), (6.59), (6.69), and (6.71) with (6.68) for $\hat{\rho}_{1}, \hat{v}_{i 1}, \hat{T}_{1}$, and $\hat{p}_{1}$ in [56] (ignore the subscript $V$ there) because it is proved in [61] that the non-Navier-Stokes terms do not appear up to this order. [Because of the general curvilinear coordinate system used in $[61,56,57]$, the re-expansion is extremely complicated. We must say that we have confirmed only the leading-order equations $(6.52),(6.53)$, (6.58), and (6.60) with (6.57) in [56] by the direct re-expansion]. These are the (compressible) viscous boundary-layer equations re-expanded in $\sqrt{\varepsilon}$ that should be matched with the Euler set of equations in the bulk of the gas (note that $z$ here is the same as $y$ in [56]). If we use the expansions in the slip boundary conditions (118) and take the terms of $O(1)$ and $O(\sqrt{\varepsilon})$, we obtain the no-slip condition (6.50) and the slip boundary condition (6.99) in [56], which are for the viscous boundary-layer equations. Here, we should note that a part of the term containing $c_{v}^{I}$ in $(118 \mathrm{~b})$ and the term containing $c_{T}^{I I}$ in $(118 \mathrm{c})$ are upgraded to the terms of $O(\sqrt{\varepsilon})$ because of the relation $\partial / \partial x_{i}=(1 / \sqrt{\varepsilon}) n_{i} \partial / \partial z+O(1)$; more specifically, $\varepsilon\left(\partial \hat{v}_{j 0} / \partial x_{i}\right) n_{i} t_{j}=\varepsilon\left[\partial\left(\hat{v}_{j 0} t_{j}\right) / \partial x_{i}\right] n_{i}=\sqrt{\varepsilon} \partial\left(\hat{v}_{j 0} t_{j}\right) / \partial z+O(\varepsilon)$ and $\varepsilon\left(\partial \hat{T}_{0} / \partial x_{i}\right) n_{i}=\sqrt{\varepsilon}\left(\partial \hat{T}_{0} / \partial z\right)+O(\varepsilon)$. The term containing $c_{v}^{I I}$, which also contains the derivative $\left(\partial / \partial x_{i}\right) n_{i}$, remains to be of $O(\varepsilon)$ because $\hat{v}_{i 0} n_{i}=0$ [or $\left.\hat{v}_{i} n_{i}=O(\sqrt{\varepsilon})\right]$ inside the viscous boundary layer. In this way, these upgraded terms in the boundary conditions give rise to the velocity and temperature fields of $O(\sqrt{\varepsilon})$. This is the reason why the expansion parameter is $\sqrt{\varepsilon}$, not $\varepsilon$ itself. On the other hand, the Navier-Stokes equations (50) automatically transform into the boundary-layer equations near the boundary in the situation where the viscous boundary layer appears. In summary, the present system composed of the Navier-Stokes equations (50) and the slip boundary conditions (118) should cover the situation considered in $[61,56,57]$ correctly.

Finally we make short remarks on the slip boundary conditions for the compressible Navier-Stokes equations derived by Coron [18]. In this reference, the slip boundary conditions are first derived for a gas between two plates (steady plane Couette flow with a temperature difference) by the correct and precise analysis, and then the mathematical properties of the solution of the compressible Navier-Stokes equations with the slip boundary conditions are discussed. As for the boundary of arbitrary shape, only a brief outline of the analysis is shown. In addition, the analysis is restricted to the time-independent case with stationary boundaries. Since the boundary is at rest, $\left(\partial \hat{v}_{i} / \partial x_{j}\right) n_{i} t_{j}$ in $(118 \mathrm{~b})$ becomes of $O(\varepsilon)$, and the term containing $c_{v}^{I}$ reduces to $\varepsilon c_{v}^{I}(1 / \hat{\rho})\left(\partial \hat{v}_{j} t_{j} / \partial x_{i}\right) n_{i}$. In fact, the slip boundary condition in [18] [(3.22)] contains this term. Therefore, if one carelessly apply the slip boundary conditions in [18] to the problems where the boundary has a tangential velocity, such as cylindrical Couette 
flow, the result is incorrect. Compared with (118c), the formula (3.23) in [18] contains an additional term proportional to $\varepsilon \partial\left(\hat{u}_{i}-\hat{u}_{j} n_{j} n_{i}\right) / \partial x_{i}$ in the notation of the present paper. However, this term can be shown to be of $O\left(\varepsilon^{2}\right)$, so that there is no need to include this term. We should also emphasize that the numerical values of the slip coefficients, i.e., $c_{v}^{I}, c_{T}^{I}, c_{v}^{I I}$, and $c_{T}^{I I}$, are not included in [18]. This means that it is not possible to apply the result in [18] to the practical problems immediately.

\subsubsection{Macroscopic Quantities Inside the Knudsen Layer}

From (93) and (75), (76), and (80), we have

$$
\int \mathscr{C}_{\mathrm{wn}} \phi E\left(\mathscr{C}_{\mathrm{w}}\right) d \mathscr{C}_{\mathrm{w}}=O(\varepsilon)
$$

By the similar procedure, or by integrating (81) multiplied by $\mathscr{C}_{\mathrm{w} i} E\left(\mathscr{C}_{\mathrm{w}}\right)$ and $\mathscr{C}_{\mathrm{w}}^{2} E\left(\mathscr{C}_{\mathrm{w}}\right)$ over the whole space of $\mathscr{C}_{\mathrm{w}}$ and taking into account the fact that $\phi \rightarrow 0$ as $y \rightarrow \infty$, we obtain

$$
\int \mathscr{C}_{\mathrm{w} i} \mathscr{C}_{\mathrm{wn}} \phi E\left(\mathscr{C}_{\mathrm{w}}\right) d \mathscr{C}_{\mathrm{w}}=O(\varepsilon), \quad \int \mathscr{C}_{\mathrm{w}}^{2} \mathscr{C}_{\mathrm{wn}} \phi E\left(\mathscr{C}_{\mathrm{w}}\right) d \mathscr{C}_{\mathrm{w}}=O(\varepsilon)
$$

From (82b), (82e), and (82f), the relations (119) and (120) indicate that $\hat{v}_{\mathrm{Ki}}^{(1)} n_{i}, \hat{p}_{\mathrm{K} i j}^{(1)} n_{j}$, and $\hat{q}_{\mathrm{K} i}^{(1)} n_{i}$ are all of $O(\varepsilon)$. Other components of $\hat{h}_{\mathrm{K}}^{(1)}$ are obtained by substituting (104) into (82). We summarize the result of the Knudsen-layer correction of the macroscopic quantities $\hat{h}_{\mathrm{K}}=\hat{h}_{\mathrm{K}}^{(1)} \varepsilon+O\left(\varepsilon^{2}\right)[(58)]$ neglecting the terms of $O\left(\varepsilon^{2}\right)$, that is,

$$
\begin{aligned}
& \hat{v}_{\mathrm{K} i} n_{i}=0 \\
& \hat{v}_{\mathrm{K} i} t_{i}=\varepsilon Y_{v}(y) \frac{1}{\hat{\rho}}\left(\frac{\partial \hat{v}_{i}}{\partial x_{j}}+\frac{\partial \hat{v}_{j}}{\partial x_{i}}\right) n_{i} t_{j}+\varepsilon Y_{T}(y) \frac{1}{\hat{\rho} \hat{T}_{\mathrm{w}}^{1 / 2}} \frac{\partial \hat{T}}{\partial x_{i}} t_{i} \\
& \hat{\rho}_{\mathrm{K}}=\varepsilon \Omega_{v}(y) \frac{1}{\hat{T}_{\mathrm{w}}^{1 / 2}} \frac{\partial \hat{v}_{i}}{\partial x_{j}} n_{i} n_{j}+\varepsilon \Omega_{T}(y) \frac{1}{\hat{T}_{\mathrm{w}}} \frac{\partial \hat{T}}{\partial x_{i}} n_{i}, \\
& \hat{T}_{\mathrm{K}}=\varepsilon \Theta_{v}(y) \frac{\hat{T}_{\mathrm{w}}^{1 / 2}}{\hat{\rho}} \frac{\partial \hat{v}_{i}}{\partial x_{j}} n_{i} n_{j}+\varepsilon \Theta_{T}(y) \frac{1}{\hat{\rho}} \frac{\partial \hat{T}}{\partial x_{i}} n_{i}, \\
& \hat{p}_{\mathrm{K} i j} n_{j}=0, \\
& \hat{p}_{\mathrm{K} i j} t_{j}=\varepsilon \Pi_{v}(y) \hat{T}_{\mathrm{w}}^{1 / 2} \frac{\partial \hat{v}_{j}}{\partial x_{k}} n_{j} n_{k} t_{i}+\varepsilon \Pi_{T}(y) \frac{\partial \hat{T}}{\partial x_{j}} n_{j} t_{i}, \\
& \hat{q}_{\mathrm{K} i} n_{i}=0, \\
& \hat{q}_{\mathrm{K} i} t_{i}=\varepsilon H_{v}(y) \hat{T}_{\mathrm{w}}\left(\frac{\partial \hat{v}_{i}}{\partial x_{j}}+\frac{\partial \hat{v}_{j}}{\partial x_{i}}\right) n_{i} t_{j}+\varepsilon H_{T}(y) \hat{T}_{\mathrm{w}}^{1 / 2} \frac{\partial \hat{T}}{\partial x_{i}} t_{i},
\end{aligned}
$$

where

$$
Y_{\gamma}(y)=\frac{1}{2} \int\left(\mathscr{C}_{\mathrm{w}}^{2}-\mathscr{C}_{\mathrm{wn}}^{2}\right) \phi_{\gamma}^{I} E\left(\mathscr{C}_{\mathrm{w}}\right) d \mathscr{C}_{\mathrm{w}}, \quad(\gamma=v, T),
$$




$$
\begin{aligned}
& \Omega_{\gamma}(y)=\int \phi_{\gamma}^{I I} E\left(\mathscr{C}_{\mathrm{w}}\right) d \mathscr{C}_{\mathrm{w}}, \quad(\gamma=v, T), \\
& \Theta_{\gamma}(y)=\frac{2}{3} \int\left(\mathscr{C}_{\mathrm{w}}^{2}-\frac{3}{2}\right) \phi_{\gamma}^{I I} E\left(\mathscr{C}_{\mathrm{w}}\right) d \mathscr{C}_{\mathrm{w}}, \quad(\gamma=v, T), \\
& \Pi_{\gamma}(y)=\frac{3}{2}\left[\Omega_{\gamma}(y)+\Theta_{\gamma}(y)\right], \quad(\gamma=v, T), \\
& H_{\gamma}(y)=\frac{1}{2} \int\left(\mathscr{C}_{\mathrm{w}}^{2}-\mathscr{C}_{\mathrm{wn}}^{2}\right)\left(\mathscr{C}_{\mathrm{w}}^{2}-\frac{5}{2}\right) \phi_{\gamma}^{I} E\left(\mathscr{C}_{\mathrm{w}}\right) d \mathscr{C}_{\mathrm{w}}, \quad(\gamma=v, T) .
\end{aligned}
$$

In (121), $\hat{\rho}, \hat{v}_{i}$, and $\hat{T}$, which belong to the Chapman-Enskog solution, and their derivatives are all evaluated on the boundary.

For hard-sphere molecules and the diffuse reflection, $\left[y, Y_{v}(y), Y_{T}(y), H_{v}(y)\right.$, $\left.H_{T}(y)\right]($ here $)=\left[x_{1}, S\left(x_{1}\right), C\left(x_{1}\right), H_{A}\left(x_{1}\right), H_{B}\left(x_{1}\right)\right]($ in $[36])=\left[\eta,-Y_{0}(\eta),-(1 / 2) Y_{1}(\eta)\right.$,

$\left.H_{A}(\eta), H_{B}(\eta)\right]$ (in [57]; see Table 3.2 in [57]); [y, $\left.\Omega_{v}(y), \Theta_{v}(y)\right]$ (here) $=\left[\eta, \Omega_{5}^{(0)}(\eta)\right.$, $\left.\Theta_{5}^{(0)}(\eta)\right]$ (in [25]); [y, $\left.\Omega_{T}(y), \Theta_{T}(y)\right]$ (here) $=\left[x_{1}, \Omega\left(x_{1}\right), \Theta\left(x_{1}\right)\right]$ (in [62]) $=[\eta$, $\Omega_{1}(\eta), \Theta_{1}(\eta)$ ] (in [57]; see Table 3.2 in [57]).

For the BGK model and the diffuse reflection, $y$ (here) $=\eta$ (in [57]) and $\left[Y_{v}(y), Y_{T}(y), \Omega_{v}(y), \Theta_{v}(y), \Omega_{T}(y), \Theta_{T}(y), H\right.$ (here) $=\left[\hat{T}_{\mathrm{w}}^{1 / 2} / \hat{A}_{c}\left(\hat{T}_{\mathrm{w}}\right)\right] \times\left[-Y_{0}(\eta),-(1 / 2) Y_{1}(\eta),(4 / 3) \Omega_{6}(\eta),(4 / 3) \Theta_{6}(\eta), \Omega_{1}(\eta), \Theta_{1}(\eta), H_{A}(\eta), H_{B}(\eta)\right]$ (in [57]; see Table 3.3 in [57]).

\section{Navier-Stokes Equations and Slip Boundary Condi- tions in Dimensional Form}

In this section, we rewrite the compressible Navier-Stokes equations (50) and the slip boundary conditions (118) in the original dimensional form.

With the help of (12), (13), and (46), (50) can be transformed to the following dimensional Navier-Stokes equations:

$$
\begin{aligned}
& \frac{\partial \rho}{\partial t}+\frac{\partial \rho v_{j}}{\partial X_{j}}=0 \\
& \frac{\partial \rho v_{i}}{\partial t}+\frac{\partial \rho v_{i} v_{j}}{\partial X_{j}}=-\frac{\partial p}{\partial X_{i}}+\frac{\partial}{\partial X_{j}}\left[\mu(T)\left(\frac{\partial v_{i}}{\partial X_{j}}+\frac{\partial v_{j}}{\partial X_{i}}-\frac{2}{3} \frac{\partial v_{k}}{\partial X_{k}} \delta_{i j}\right)\right] \\
& \frac{\partial}{\partial t}\left[\rho\left(\frac{3}{2} R T+\frac{1}{2} v_{j}^{2}\right)\right]+\frac{\partial}{\partial X_{j}}\left[\rho v_{j}\left(\frac{5}{2} R T+\frac{1}{2} v_{k}^{2}\right)\right] \\
& \quad=\frac{\partial}{\partial X_{j}}\left[\lambda(T) \frac{\partial T}{\partial X_{j}}\right]+\frac{\partial}{\partial X_{j}}\left[\mu(T) v_{i}\left(\frac{\partial v_{i}}{\partial X_{j}}+\frac{\partial v_{j}}{\partial X_{i}}-\frac{2}{3} \frac{\partial v_{k}}{\partial X_{k}} \delta_{i j}\right)\right]
\end{aligned}
$$

where $p=R \rho T$ [(1d)].

Using (12) and (13) and eliminating $\varepsilon$ with the help of (46), we can transform (118) to the following dimensional slip boundary conditions:

$$
\begin{aligned}
& \left(v_{i}-v_{\mathrm{w} i}\right) n_{i}=0 \\
& \left(v_{i}-v_{\mathrm{w} i}\right) t_{i}=\frac{\sqrt{2}}{R^{1 / 2}} a_{v}^{I} \frac{\mu\left(T_{\mathrm{w}}\right)}{\rho T_{\mathrm{w}}^{1 / 2}}\left(\frac{\partial v_{i}}{\partial X_{j}}+\frac{\partial v_{j}}{\partial X_{i}}\right) n_{i} t_{j}+\frac{4}{5 R} a_{T}^{I} \frac{\lambda\left(T_{\mathrm{w}}\right)}{\rho T_{\mathrm{w}}} \frac{\partial T}{\partial X_{i}} t_{i}
\end{aligned}
$$




$$
T-T_{\mathrm{w}}=\frac{1}{R} a_{v}^{I I} \frac{\mu\left(T_{\mathrm{w}}\right)}{\rho} \frac{\partial v_{i}}{\partial X_{j}} n_{i} n_{j}+\frac{2 \sqrt{2}}{5 R^{3 / 2}} a_{T}^{I I} \frac{\lambda\left(T_{\mathrm{w}}\right)}{\rho T_{\mathrm{w}}^{1 / 2}} \frac{\partial T}{\partial X_{i}} n_{i},
$$

where $\mu\left(T_{\mathrm{w}}\right)$ and $\lambda\left(T_{\mathrm{w}}\right)$ are the viscosity and thermal conductivity at temperature $T_{\mathrm{w}}$, respectively [cf. (46), (48), and (49)], and $a_{v}^{I}, a_{T}^{I}, a_{v}^{I I}$, and $a_{T}^{I I}$ are defined by

$$
\begin{array}{ll}
a_{v}^{I}=\left(\frac{T_{\mathrm{w}}}{T_{0}}\right)^{1 / 2} \frac{c_{v}^{I}}{\Gamma_{1}\left(T_{\mathrm{w}} / T_{0}\right)}, & a_{T}^{I}=\left(\frac{T_{\mathrm{w}}}{T_{0}}\right)^{1 / 2} \frac{c_{T}^{I}}{\Gamma_{2}\left(T_{\mathrm{w}} / T_{0}\right)}, \\
a_{v}^{I I}=\left(\frac{T_{\mathrm{w}}}{T_{0}}\right)^{1 / 2} \frac{c_{v}^{I I}}{\Gamma_{1}\left(T_{\mathrm{w}} / T_{0}\right)}, & a_{T}^{I I}=\left(\frac{T_{\mathrm{w}}}{T_{0}}\right)^{1 / 2} \frac{c_{T}^{I I}}{\Gamma_{2}\left(T_{\mathrm{w}} / T_{0}\right)} .
\end{array}
$$

In (124), the quantities $\rho, v_{i}, T$, and their derivatives are all evaluated on the boundary. The coefficients $a_{v}^{I}, a_{T}^{I}, a_{v}^{I I}$, and $a_{T}^{I I}$, which seemingly depend on $T_{\mathrm{w}}$ and $T_{0}$, should be constants. In fact, from (47) and (114)-(117), we have the following expressions of these coefficients in the case of diffuse reflection:

- For hard-sphere molecules

$$
a_{v}^{I}=0.98733, \quad a_{T}^{I}=0.33628, \quad a_{v}^{I I}=0.36185, \quad a_{T}^{I I}=1.24859 .
$$

- For the BGK model

$$
a_{v}^{I}=1.01619, \quad a_{T}^{I}=0.38316, \quad a_{v}^{I I}=0.44045, \quad a_{T}^{I I}=1.30272 .
$$

As we can see from (126) and (127), the coefficients do not differ much between these completely different collision models.

The initial condition for (123) is given by

$$
\rho=\rho_{0}, \quad \boldsymbol{v}=0, \quad T=T_{0}, \quad \text { at } t=0,
$$

corresponding to (51), under assumption (iv) in Sect. 2, or

$$
\rho=\rho_{\text {ini }}(\boldsymbol{X}), \quad \boldsymbol{v}=\boldsymbol{v}_{\text {ini }}(\boldsymbol{X}), \quad T=T_{\text {ini }}(\boldsymbol{X}), \quad \text { at } t=0,
$$

corresponding to (103), in more general case without assumption (iv), where $\rho_{\mathrm{ini}}, \boldsymbol{v}_{\mathrm{ini}}$, and $T_{\text {ini }}$ are the density, flow velocity, and temperature obtained from the initial distribution $f_{\text {ini }}$ with the help of (1a)-(1c). (See the comments in the last two paragraphs of Sect. 5.2.4.)

\section{Concluding Remarks}

In the present study, we considered a fully nonlinear setting, which corresponds to the case of finite Mach numbers. We started with the first-order Chapman-Enskog solution as an ansatz for the compressible Navier-Stokes equations and derived their slip 
boundary conditions by the analysis of the Knudsen layer. This setting is the same as that in the nonlinear asymptotic theory by Sone et al. [61] though the analysis there is restricted to the time-independent problems. In this reference, a systematic asymptotic expansion, based on the Hilbert-type expansion with $\sqrt{\varepsilon}$ (not $\varepsilon$ ) as the expansion parameter, is carried out, and the fluid-dynamic equations and their slip boundary conditions, together with the corrections inside the Knudsen layer, are obtained. The fluid-dynamic equations are split into two types: The Euler equations in the bulk of the gas, and the viscous boundary-layer equations near the boundary. As we have seen in Sect. 5.3.4, the fact that the expansion parameter should be $\sqrt{\varepsilon}$ is closely related to the appearance of the viscous boundary layer whose thickness (in the dimensionless $\boldsymbol{x}$ space) is of the order of $\sqrt{\varepsilon}$.

The asymptotic theory in [61] is perfect theoretically. However, in addition to its restriction to the time-independent flows, its application to numerical computation of practical problems is not straightforward because of the matching process between the Euler and viscous boundary layer equations. In addition, the theory is not applicable to problems in which the viscous boundary layer is not formed on the whole boundary. On the other hand, the theory guarantees that the Navier-Stokes equations with the correct slip boundary conditions provide correct overall solution up to the order of $\sqrt{\varepsilon}$. From these facts, we were motivated to use the single set of the Navier-Stokes equations with the slip boundary conditions in order to establish a more flexible system.

The analysis of the present paper is based on the method of Knudsen layers established by Sone $[53,54,59,55,60,61,56,57]$, and its contents are the revisit to the work by Coron [18] for the same problem. The difference between [18] and the present paper, which has been discussed in Sect. 5.3.4, is summarized as follows: (i) In [18], steady flows are considered and the boundaries are assumed to be at rest, whereas timedependent problems with arbitrarily moving boundaries are considered in the present study. The formulas of the slip boundary conditions presented in [18] are seemingly different from (118). However, taking into account the fact that the boundary is at rest in [18] and that some terms in the slip boundary conditions in [18] can be estimated as of the order higher than $\varepsilon$, we can show that the slip condition in [18] can be recovered from (118). (ii) The reference [18] does not give the explicit values of the coefficients (the so-called slip coefficients) in the formulas of the slip boundary conditions, so that it is hard to apply the formulas immediately to numerical computation of practical problems.

In the present paper, we derived the slip boundary conditions for the Navier-Stokes equations in a clear way and give their formulas with explicit numerical values for hardsphere molecules and for the BGK model under the diffuse reflection boundary conditions. These formulas should facilitate the correct application of the Navier-Stokes equations to the practical problems with moving boundaries of arbitrary shape in the slip-flow regime. Since the process of analysis, in particular, the reduction of the originally nonlinear problem to the linearized Knudsen-layer problems, has been shown explicitly, its extension to different intermolecular potentials and boundary conditions is a straightforward task.

Finally, we comment on the order of the slip boundary conditions. The first-order slip boundary conditions (118) are consistent with the compressible Navier-Stokes equations (50) because they are constructed in such a way that the Boltzmann equa- 
tion and its boundary condition are satisfied up to the order of $\varepsilon$. If we try to derive the second-order slip boundary conditions, we have to satisfy the Boltzmann equation as well as its boundary condition up to the order of $\varepsilon^{2}$. For this purpose, we first need the higher-order correction to the first-order Chapman-Enskog solution. If we take the second-order Chapman-Enskog solution, however, it leads to the Burnett equations [16], which have unfavorable properties [10] and contain higher-order derivatives. This is the reason why we considered in this paper the overall solution as the sum of the firstorder Chapman-Enskog solution and the remainder without touching the higher order solution. One of the potential approaches to proceed to the $\varepsilon^{2}$-order would be to construct the overall solution as the sum of the first-order Chapman-Enskog solution and its correction of the order of $\varepsilon^{2}$ and derive the fluid-dynamic equations and the secondorder slip boundary conditions for the correction part by a similar analysis as in the present paper.

A cknowledgements.

The present work was supported in part by the grant-in-aid for scientific research No. 26630051 from JSPS. A part of the work was performed while one of the authors (K.A.) was visiting at Institut de Mathématiques de Bordeaux. He wishes to thank it for its kind invitation and hospitality. He also thanks National Center for Theoretical Sciences, National Taiwan University and Department of Mathematics, National Cheng Kung University for their support and hospitality.

\section{A Derivation of (72): Outline}

Let us define the following column vectors $\boldsymbol{a}$ and $\boldsymbol{b}$ of seven components:

$$
\boldsymbol{a}={ }^{\mathrm{t}}\left(\hat{t}, x_{1}, x_{2}, x_{3}, \zeta_{1}, \zeta_{2}, \zeta_{3}\right), \quad \boldsymbol{b}={ }^{\mathrm{t}}\left(\tilde{t}, \eta, \chi_{1}, \chi_{2}, \zeta_{\mathrm{w} 1}, \zeta_{\mathrm{w} 2}, \zeta_{\mathrm{w} 3}\right),
$$

where ${ }^{\mathrm{t}}$ indicates the transpose operation. Then, the Jacobian matrix of the transformation from $(\hat{t}, \boldsymbol{x}, \boldsymbol{\zeta})$ to $\left(\tilde{t}, \eta, \chi_{1}, \chi_{2}, \boldsymbol{\zeta}_{\mathrm{w}}\right)$, which we denote by $\mathrm{A}=\left[A_{i j}\right]$, can be expressed as

$$
\mathrm{A}=\frac{\partial\left(\tilde{t}, \eta, \chi_{1}, \chi_{2}, \zeta_{\mathrm{w} 1}, \zeta_{\mathrm{w} 2}, \zeta_{\mathrm{w} 3}\right)}{\partial\left(\hat{t}, x_{1}, x_{2}, x_{3}, \zeta_{1}, \zeta_{2}, \zeta_{3}\right)}=\left[\frac{\partial \boldsymbol{b}}{\partial \hat{t}}, \frac{\partial \boldsymbol{b}}{\partial x_{1}}, \frac{\partial \boldsymbol{b}}{\partial x_{2}}, \frac{\partial \boldsymbol{b}}{\partial x_{3}}, \frac{\partial \boldsymbol{b}}{\partial \zeta_{1}}, \frac{\partial \boldsymbol{b}}{\partial \zeta_{2}}, \frac{\partial \boldsymbol{b}}{\partial \zeta_{3}}\right],
$$

and that of the inverse transformation, which we denote by $\mathrm{B}=\left[B_{i j}\right]$, as

$$
\mathrm{B}=\frac{\partial\left(\hat{t}, x_{1}, x_{2}, x_{3}, \zeta_{1}, \zeta_{2}, \zeta_{3}\right)}{\partial\left(\tilde{t}, \eta, \chi_{1}, \chi_{2}, \zeta_{\mathrm{w} 1}, \zeta_{\mathrm{w} 2}, \zeta_{\mathrm{w} 3}\right)}=\left[\frac{\partial \boldsymbol{a}}{\partial \tilde{t}}, \frac{\partial \boldsymbol{a}}{\partial \eta}, \frac{\partial \boldsymbol{a}}{\partial \chi_{1}}, \frac{\partial \boldsymbol{a}}{\partial \chi_{2}}, \frac{\partial \boldsymbol{a}}{\partial \zeta_{\mathrm{w} 1}}, \frac{\partial \boldsymbol{a}}{\partial \zeta_{\mathrm{w} 2}}, \frac{\partial \boldsymbol{a}}{\partial \zeta_{\mathrm{w} 3}}\right]
$$

In order to obtain (72), we need to calculate some components of $A$, which is not straightforward from (64). Therefore, we calculate them indirectly using the fact that $A=B^{-1}$. Because each component of the matrix $B$ can be calculated immediately 
from (64):

$$
\begin{aligned}
& \frac{\partial \hat{t}}{\partial \tilde{t}}=1, \quad \frac{\partial \hat{t}}{\partial \eta}=\frac{\partial \hat{t}}{\partial \chi_{1}}=\frac{\partial \hat{t}}{\partial \chi_{2}}=\frac{\partial \hat{t}}{\partial \zeta_{\mathrm{w} i}}=0, \\
& \frac{\partial x_{i}}{\partial \tilde{t}}=\hat{v}_{\mathrm{w} i}+\varepsilon \eta \frac{\partial n_{i}}{\partial \tilde{t}}, \quad \frac{\partial x_{i}}{\partial \eta}=\varepsilon n_{i}, \\
& \frac{\partial x_{i}}{\partial \chi_{1}}=\frac{\partial x_{\mathrm{w} i}}{\partial \chi_{1}}+\varepsilon \eta \frac{\partial n_{i}}{\partial \chi_{1}}, \quad \frac{\partial x_{i}}{\partial \chi_{2}}=\frac{\partial x_{\mathrm{w} i}}{\partial \chi_{2}}+\varepsilon \eta \frac{\partial n_{i}}{\partial \chi_{2}}, \quad \frac{\partial x_{i}}{\partial \zeta_{\mathrm{w} j}}=0, \\
& \frac{\partial \zeta_{i}}{\partial \tilde{t}}=\frac{\partial \hat{v}_{\mathrm{w} i}}{\partial \tilde{t}}, \quad \frac{\partial \zeta_{i}}{\partial \eta}=0, \quad \frac{\partial \zeta_{i}}{\partial \chi_{1}}=\frac{\partial \hat{v}_{\mathrm{w} i}}{\partial \chi_{1}}, \quad \frac{\partial \zeta_{i}}{\partial \chi_{2}}=\frac{\partial \hat{v}_{\mathrm{w} i}}{\partial \chi_{2}}, \quad \frac{\partial \zeta_{i}}{\partial \zeta_{\mathrm{w} j}}=\delta_{i j},
\end{aligned}
$$

each component of the matrix A can be calculated, in principle, by the usual formula of the inverse matrix. Here, we should note that, because of (133), many components of $B$ vanishes, so that the determinant of $B$, denoted by $\operatorname{det} B$, can be obtained in a simple form. That is, if we interpret $\boldsymbol{x}, \boldsymbol{x}_{\mathrm{w}}$, and $\boldsymbol{n}$ in the matrix representation as the column vectors $\boldsymbol{x}={ }^{\mathrm{t}}\left(x_{1}, x_{2}, x_{3}\right), \boldsymbol{x}_{\mathrm{w}}={ }^{\mathrm{t}}\left(x_{\mathrm{w} 1}, x_{\mathrm{w} 2}, x_{\mathrm{w} 3}\right)$, and $\boldsymbol{n}={ }^{t}\left(n_{1}, n_{2}, n_{3}\right)$, then we have

$$
\begin{aligned}
\operatorname{det} \mathrm{B} & =\operatorname{det}\left[\frac{\partial \boldsymbol{x}}{\partial \eta}, \frac{\partial \boldsymbol{x}}{\partial \chi_{1}}, \frac{\partial \boldsymbol{x}}{\partial \chi_{2}}\right] \\
& =\operatorname{det}\left[\varepsilon \boldsymbol{n}, \frac{\partial \boldsymbol{x}_{\mathrm{w}}}{\partial \chi_{1}}+\varepsilon \eta \frac{\partial \boldsymbol{n}}{\partial \chi_{1}}, \frac{\partial \boldsymbol{x}_{\mathrm{w}}}{\partial \chi_{2}}+\varepsilon \eta \frac{\partial \boldsymbol{n}}{\partial \chi_{2}}\right] \\
& =\varepsilon \operatorname{det}\left[\boldsymbol{n}, \frac{\partial \boldsymbol{x}_{\mathrm{w}}}{\partial \chi_{1}}, \frac{\partial \boldsymbol{x}_{\mathrm{w}}}{\partial \chi_{2}}\right]+O\left(\varepsilon^{2}\right) \\
& =\boldsymbol{\varepsilon} \boldsymbol{n} \cdot\left(\frac{\partial \boldsymbol{x}_{\mathrm{w}}}{\partial \chi_{1}} \times \frac{\partial \boldsymbol{x}_{\mathrm{w}}}{\partial \chi_{2}}\right)+O\left(\varepsilon^{2}\right) .
\end{aligned}
$$

Here, we note from (63) that

$$
\frac{\partial \boldsymbol{x}_{\mathrm{w}}}{\partial \chi_{1}} \times \frac{\partial \boldsymbol{x}_{\mathrm{w}}}{\partial \chi_{2}}=\delta\left|\frac{\partial \boldsymbol{x}_{\mathrm{w}}}{\partial \chi_{1}} \times \frac{\partial \boldsymbol{x}_{\mathrm{W}}}{\partial \chi_{2}}\right| \boldsymbol{n}
$$

where $\delta=1$ when $\left(\partial \boldsymbol{x}_{\mathrm{w}} / \partial \chi_{1}\right) \times\left(\partial \boldsymbol{x}_{\mathrm{w}} / \partial \chi_{2}\right)$ and $\boldsymbol{n}$ are in the same direction, and $\boldsymbol{\delta}=$ -1 when they are in the opposite direction.

Let us calculate $\partial \eta / \partial \hat{t}$, which is the $(2,1)$ component of A. If we denote by $C_{m, n}$ the $(m, n)$ cofactor of $\mathrm{B}$ and interpret $\hat{\boldsymbol{v}}_{\mathrm{w}}$ in the matrix representation as the column 
vector $\hat{\boldsymbol{v}}_{\mathrm{w}}={ }^{\mathrm{t}}\left(\hat{v}_{\mathrm{w} 1}, \hat{v}_{\mathrm{w} 2}, \hat{v}_{\mathrm{w} 3}\right)$, then we obtain, with the help of (134) and (135),

$$
\begin{aligned}
\frac{\partial \eta}{\partial \hat{t}} & =C_{1,2}(\operatorname{det} \mathrm{B})^{-1} \\
& =-\operatorname{det}\left[\frac{\partial \boldsymbol{x}}{\partial \tilde{t}}, \frac{\partial \boldsymbol{x}}{\partial \chi_{1}}, \frac{\partial \boldsymbol{x}}{\partial \chi_{2}}\right](\operatorname{det} \mathrm{B})^{-1} \\
& =-\operatorname{det}\left[\hat{\boldsymbol{v}}_{\mathrm{w}}+\varepsilon \eta \frac{\partial \boldsymbol{n}}{\partial \tilde{t}}, \frac{\partial \boldsymbol{x}_{\mathrm{w}}}{\partial \chi_{1}}+\varepsilon \eta \frac{\partial \boldsymbol{n}}{\partial \chi_{1}}, \frac{\partial \boldsymbol{x}_{\mathrm{w}}}{\partial \chi_{2}}+\varepsilon \eta \frac{\partial \boldsymbol{n}}{\partial \chi_{2}}\right](\operatorname{det} \mathrm{B})^{-1} \\
& =\left\{-\operatorname{det}\left[\hat{\boldsymbol{v}}_{\mathrm{w}}, \frac{\partial \boldsymbol{x}_{\mathrm{w}}}{\partial \chi_{1}}, \frac{\partial \boldsymbol{x}_{\mathrm{w}}}{\partial \chi_{2}}\right]+O(\varepsilon)\right\}(\operatorname{det} \mathrm{B})^{-1} \\
& =\left[-\hat{\boldsymbol{v}}_{\mathrm{w}} \cdot\left(\frac{\partial \boldsymbol{x}_{\mathrm{w}}}{\partial \chi_{1}} \times \frac{\partial \boldsymbol{x}_{\mathrm{w}}}{\partial \chi_{2}}\right)+O(\varepsilon)\right](\operatorname{det} \mathrm{B})^{-1} \\
& =-\frac{1}{\varepsilon} \hat{\boldsymbol{v}}_{\mathrm{w}} \cdot \boldsymbol{n}+O(1) .
\end{aligned}
$$

Similarly, $\partial \eta / \partial x_{i}$, which is the $(2, i+1)$ component of $\mathrm{A}$, can be calculated as follows:

$$
\begin{aligned}
\frac{\partial \eta}{\partial x_{i}} & =C_{i+1,2}(\operatorname{det} \mathrm{B})^{-1} \\
& =\left(\frac{\partial \boldsymbol{x}}{\partial \chi_{1}} \times \frac{\partial \boldsymbol{x}}{\partial \chi_{2}}\right)_{i}(\operatorname{det} \mathrm{B})^{-1} \\
& =\left[\left(\frac{\partial \boldsymbol{x}_{\mathrm{w}}}{\partial \chi_{1}} \times \frac{\partial \boldsymbol{x}_{\mathrm{w}}}{\partial \chi_{2}}\right)_{i}+O(\varepsilon)\right](\operatorname{det} \mathrm{B})^{-1} \\
& =\frac{1}{\varepsilon} n_{i}+O(1) .
\end{aligned}
$$

We omit the calculation for the other quantities in (72) that turn out to be of $O(1)$.

\section{B Derivation of (88): Outline}

Since $\hat{\boldsymbol{v}}_{\mathrm{B}}=\hat{\boldsymbol{v}}_{\mathrm{w}}+\varepsilon \check{\boldsymbol{v}}$ and $\hat{T}_{\mathrm{B}}=\hat{T}_{\mathrm{w}}+\varepsilon \check{T}[(59)], \hat{f}^{(0)}$ on the boundary is expressed as

$$
\hat{f}_{\mathrm{B}}^{(0)}=\frac{\hat{\rho}_{\mathrm{B}}}{\left[\pi\left(\hat{T}_{\mathrm{w}}+\varepsilon \check{T}\right)\right]^{3 / 2}} \exp \left(-\frac{\left|\boldsymbol{\zeta}-\hat{\boldsymbol{v}}_{\mathrm{w}}-\varepsilon \check{\boldsymbol{v}}\right|^{2}}{\hat{T}_{\mathrm{w}}+\varepsilon \check{T}}\right) .
$$

Expanding this expression in $\varepsilon$, retaining the terms of $O(\varepsilon)$, and using $\mathscr{C}_{\text {w }}$ of (74), we obtain (88a).

If we take into account that $\hat{f}_{\mathrm{B}}^{(0)}=\hat{f}_{\mathrm{w}}[1+O(\varepsilon)]$ and $\Psi_{\mathrm{B}}=\Psi_{\mathrm{w}}+O(\varepsilon)$ for $\hat{f}^{(1)}=$ $\hat{f}^{(0)} \Psi$ on the boundary, we immediately get (88b).

By using (88a) with (75), the term containing $\hat{f}^{(0)}$ in (87b) can be calculated as follows:

$$
-2\left(\frac{\pi}{\hat{T}_{\mathrm{W}}}\right)^{1 / 2} \int_{\left(\boldsymbol{\zeta}-\hat{\boldsymbol{v}}_{\mathrm{W}}\right) \cdot \boldsymbol{n}<0}\left(\boldsymbol{\zeta}-\hat{\boldsymbol{v}}_{\mathrm{W}}\right) \cdot \boldsymbol{n} \hat{f}^{(0)} d \boldsymbol{\zeta}
$$




$$
\begin{aligned}
& =-2 \sqrt{\pi} \hat{\rho}_{\mathrm{B}} \int_{\mathscr{C}_{\mathrm{wn}}<0} \mathscr{C}_{\mathrm{wn}}\left\{1+\varepsilon\left[2 \mathscr{C}_{\mathrm{w} i} \frac{\check{v}_{i}}{\hat{T}_{\mathrm{w}}^{1 / 2}}+\left(\mathscr{C}_{\mathrm{w}}^{2}-\frac{3}{2}\right) \frac{\check{T}}{\hat{T}_{\mathrm{w}}}\right]\right\} E\left(\mathscr{C}_{\mathrm{w}}\right) d \mathscr{C}_{\mathrm{w}}+O\left(\varepsilon^{2}\right) \\
& =\hat{\rho}_{\mathrm{B}}\left[1+\varepsilon\left(-\sqrt{\pi} \frac{\check{v}_{i}}{\hat{T}_{\mathrm{w}}^{1 / 2}} n_{i}+\frac{1}{2} \frac{\check{T}}{\hat{T}_{\mathrm{w}}}\right)\right]+O\left(\varepsilon^{2}\right)
\end{aligned}
$$

Making use of (88b) with (75) and (89a) and taking (35) into account, we can transform the term containing $\hat{f}^{(1)}$ in (87b) as

$$
\begin{aligned}
-2\left(\frac{\pi}{\hat{T}_{\mathrm{w}}}\right)^{1 / 2} \int_{\left(\boldsymbol{\zeta}-\hat{v}_{\mathrm{w}}\right) \cdot \boldsymbol{n}<0}\left(\boldsymbol{\zeta}-\hat{\boldsymbol{v}}_{\mathrm{w}}\right) \cdot \boldsymbol{n} \hat{f}^{(1)} d \boldsymbol{\zeta} \\
=\sqrt{\pi} \frac{1}{\hat{T}_{\mathrm{w}}^{1 / 2}}\left[\left(\frac{\partial \hat{v}_{j}}{\partial x_{i}}\right)_{\mathrm{B}}+\left(\frac{\partial \hat{v}_{i}}{\partial x_{j}}\right)_{\mathrm{B}}\right] \\
\quad \times \int_{\mathscr{C}_{\mathrm{wn}}<0} \mathscr{C}_{\mathrm{wn}}\left(\mathscr{C}_{\mathrm{w} i} \mathscr{C}_{\mathrm{w} j}-\frac{1}{3} \mathscr{C}_{\mathrm{w}}^{2} \delta_{i j}\right) \mathscr{B}^{(0)}\left(\mathscr{C}_{\mathrm{w}}, \hat{T}_{\mathrm{w}}\right) E\left(\mathscr{C}_{\mathrm{w}}\right) d \mathscr{C}_{\mathrm{w}}+O(\varepsilon) .
\end{aligned}
$$

Here, we introduce, as at the beginning of the second paragraph in Sect. 5.3.1, two unit vectors $t$ and $s$ on the plane tangent to the boundary, which are orthogonal to each other and fixed to the boundary, i.e., $\boldsymbol{n} \cdot \boldsymbol{t}=0, \boldsymbol{n} \cdot \boldsymbol{s}=0$, and $\boldsymbol{t} \cdot \boldsymbol{s}=0$. Then, $\delta_{i j}-n_{i} n_{j}=t_{i} t_{j}+s_{i} s_{j}$ holds, and $\mathscr{C}_{\mathrm{w} i}$ can be expressed as $\mathscr{C}_{\mathrm{w} i}=\mathscr{C}_{\mathrm{wn}} n_{i}+\mathscr{C}_{\mathrm{wt}} t_{i}+\mathscr{C}_{\mathrm{ws}} s_{i}$, where $\mathscr{C}_{\mathrm{wt}}=\mathscr{C}_{\mathrm{w}} \cdot \boldsymbol{t}$ and $\mathscr{C}_{\mathrm{ws}}=\mathscr{C}_{\mathrm{w}} \cdot \boldsymbol{s}$. With these expressions, the integral in the righthand side of (140) can be calculated as

$$
\begin{aligned}
\int_{\mathscr{C}_{\mathrm{wn}}<0} \mathscr{C}_{\mathrm{wn}}\left(\mathscr{C}_{\mathrm{w} i} \mathscr{C}_{\mathrm{w} j}-\frac{1}{3} \mathscr{C}_{\mathrm{w}}^{2} \delta_{i j}\right) \mathscr{B}^{(0)}\left(\mathscr{C}_{\mathrm{w}}, \hat{T}_{\mathrm{w}}\right) E\left(\mathscr{C}_{\mathrm{w}}\right) d \mathscr{C}_{\mathrm{w}} \\
=n_{i} n_{j} \int_{\mathscr{C}_{\mathrm{wn}}<0} \mathscr{C}_{\mathrm{wn}}^{3} \mathscr{B}^{(0)}\left(\mathscr{C}_{\mathrm{w}}, \hat{T}_{\mathrm{w}}\right) E\left(\mathscr{C}_{\mathrm{w}}\right) d \mathscr{C}_{\mathrm{w}} \\
+\left(t_{i} t_{j}+s_{i} s_{j}\right) \int_{\mathscr{C}_{\mathrm{wn}}<0} \mathscr{C}_{\mathrm{wn}} \mathscr{C}_{\mathrm{wt}}^{2} \mathscr{B}^{(0)}\left(\mathscr{C}_{\mathrm{w}}, \hat{T}_{\mathrm{w}}\right) E\left(\mathscr{C}_{\mathrm{w}}\right) d \mathscr{C}_{\mathrm{w}} \\
\\
\quad-\frac{1}{3} \delta_{i j} \int_{\mathscr{C}_{\mathrm{wn}}<0} \mathscr{C}_{\mathrm{wn}} \mathscr{C}_{\mathrm{w}}^{2} \mathscr{B}^{(0)}\left(\mathscr{C}_{\mathrm{w}}, \hat{T}_{\mathrm{w}}\right) E\left(\mathscr{C}_{\mathrm{w}}\right) d \mathscr{C}_{\mathrm{w}} \\
=-\frac{1}{6 \sqrt{\pi}} \mathscr{I}_{\mathscr{B}}\left[n_{i} n_{j}-\frac{1}{2}\left(\delta_{i j}-n_{i} n_{j}\right)\right],
\end{aligned}
$$

where $\mathscr{I}_{\mathscr{B}}$ is defined in (89b). If use is made of (139) and (140) with (141) in (87b), (88c) follows.

\section{References}

[1] Aoki, K., Inamuro, T., Onishi, Y.: Slightly rarefied gas flow over a body with small accommodation coefficient. J. Phys. Soc. Japan 47, 663-671 (1979)

[2] Aoki, K., Kagaya, R., Kosuge, S., Yoshida, H.: Numerical analysis of the Taylorvortex flow of a slightly rarefied gas. In: Fan, J. (ed.) 29th International Sympo- 
sium on Rarefied Gas Dynamics 2014: AIP Conf. Proc. 1628, pp. 60-67. AIP, Melville (2014)

[3] Aoki, K., Kosuge, S., Fujiwara, T., Goudon, T.: Unsteady motion of a slightly rarefied gas caused by a plate oscillating in its normal direction. Phys. Rev. Fluids 2, 013402 (2017)

[4] Aoki, K., Yoshida, H., Nakanishi, T., Garcia, A.L.: Inverted velocity profile in the cylindrical Couette flow of a rarefied gas. Phys. Rev. E 68, 016302:1-11 (2003).

[5] Aristov, V.V.: Direct Methods for Solving the Boltzmann Equation and Study of Nonequilibrium Flows. Kluwar Academic Publishers, Dordrecht (2001)

[6] Bardos, C., Caflisch, R.E., Nicolaenko, B.: The Milne and Kramers problems for the Boltzmann equation of a hard sphere gas. Commun. Pure Appl. Math. 39, 323-352 (1986)

[7] Bhatnagar, P.L., Gross, E.P., Krook, M.: A model for collision processes in gases. I. Small amplitude processes in charged and neutral one-component systems. Phys. Rev. 94, 511-525 (1954)

[8] Bird, G.A.: Molecular Gas Dynamics. Oxford University Press, Oxford (1976)

[9] Bird, G.A.: Molecular Gas Dynamics and the Direct Simulation of Gas Flows. Oxford University Press, Oxford (1994)

[10] Bobylev, A.V.: Instabilities in the Chapman-Enskog expansion and hyperbolic Burnett equations. J. Stat. Phys. 124, 371-399 (2006).

[11] Bobylev, A.V., Rjasanow, S.: Fast deterministic method of solving the Boltzmann equation for hard spheres. Eur. J. Mech. B/Fluids 18, 869-887 (1999)

[12] Cercignani, C.: Elementary solutions of the linearized gas-dynamics Boltzmann equation and their application to the slip-flow problem. Ann. Phys. (N.Y.) 20, 219-233 (1962)

[13] Cercignani, C.: Half-space problems in the kinetic theory of gases. In: Kröner, E., Kirchgässner, K. (eds.) Trends in Applications of Pure Mathematics to Mechanics, Lecture Notes in Physics 249, pp. 35-50. Springer, Berlin (1986)

[14] Cercignani, C.: The Boltzmann Equation and Its Applications. Springer, New York (1988)

[15] Cercignani, C., Lampis, M.: Kinetic models for gas-surface interactions. Transp. Theory Stat. Phys. 1, 101-114 (1971)

[16] Chapman, S., Cowling, T.G.: The Mathematical Theory of Non-uniform Gases, 3rd edition. Cambridge Univ. Press, Cambridge (1991)

[17] Chen, I-K., Liu, T.-P., Takata, S.: Boundary singularity for thermal transpiration problem of the linearized Boltzmann equation. Arch. Rational Mech. Anal. 212, 575-595 (2014) 
[18] Coron, F.: Derivation of slip boundary conditions for the Navier-Stokes system from the Boltzmann equation. J. Stat. Phys. 54, 829-857 (1989)

[19] Filbet, F., Russo, G.: High order numerical methods for the space nonhomogeneous Boltzmann equation. J. Comput. Phys. 186, 457-480 (2003)

[20] Gamba, I.M., Tharkabhushanam, S.H.: Spectral-Lagrangian methods for collisional models of non-equilibrium statistical states. J. Comput. Phys. 228, 20122036 (2009)

[21] Garcia, R.D.M., Siewert, C.E.: Viscous-slip, thermal-slip, and temperature-jump coefficients based on the linearized Boltzmann equation (and five kinetic models) with the Cercignani-Lampis boundary condition. Eur. J. Mech. B/Fluids 29, 181191 (2010)

[22] Golse, F., Poupaud, F.: Stationary solutions of the linearized Boltzmann equation in a half-space. Math. Methods Appl. Sci. 11, 483-502 (1989)

[23] Grad, H.: Principles of the kinetic theory of gases. In: Flügge, S. (ed.) Handbuch der Physik, Band XII, pp. 205-294. Springer, Berlin (1958)

[24] Grad, H.: Singular and nonuniform limits of solutions of the Boltzmann equation. In: Bellman, R., Birkhoff, G., Abu-Shumays, I. (eds.) Transport Theory, pp. 269308. American Mathematical Society, Providence (1969)

[25] Hattori, M., Takata, S.: Second-order Knudsen-layer analysis for the generalized slip-flow theory I. Bull. Inst. Math. Acad. Sinica (New Series) 10, 423-448 (2015); see also the supplementary note in the Kyoto University Research Information Repository, http://hdl.handle.net/2433/199811

[26] Hattori, M., Takata, S.: Second-order Knudsen-layer analysis for the generalized slip-flow theory II: Curvature effects. J. Stat. Phys. 161, 1010-1036 (2015)

[27] Holway Jr., L.H.: New statistical models for kinetic theory: Methods of construction. Phys. Fluids 9, 1658-1673 (1966)

[28] Kogan, M.N.: Rarefied Gas Dynamics. Plenum, New York (1969)

[29] Li, Q., Lu, J., Sun, W.: Validity and regularization of classical half-space equations. J. Stat. Phys. 166, 398-433 (2017).

[30] Loyalka, S.K.: Temperature jump and thermal creep slip: Rigid sphere gas. Phys. Fluids A 1, 403-408 (1989)

[31] Loyalka, S.K., Ferziger, J.H.: Model dependence of the temperature slip coefficient. Phys. Fluids 11, 1668-1671 (1968)

[32] Loyalka, S.K., Hickey, K.A.: Velocity slip and defect: Hard-sphere gas. Phys. Fluids A 1, 612-614 (1989) 
[33] Loyalka, S.K., Hickey, K.A.: The Kramers problem: Velocity slip and defect for a hard sphere gas with arbitrary accommodation. Z. angew. Math. Phys. 41, 245-253 (1990)

[34] Mieussens, L.: Discrete-velocity models and numerical schemes for the Boltzmann-BGK equation in plane and axisymmetric geometries. J. Comp. Phys. 162, 429-466 (2000)

[35] Ohwada, T., Sone, Y.: Analysis of thermal stress slip flow and negative thermophoresis using the Boltzmann equation for hard-sphere molecules. Eur. J. Mech. B/Fluids 11, 389-414 (1992)

[36] Ohwada, T., Sone, Y., Aoki, K.: Numerical analysis of the shear and thermal creep flows of a rarefied gas over a plane wall on the basis of the linearized Boltzmann equation for hard-sphere molecules. Phys. Fluids A 1, 1588-1599 (1989)

[37] Onishi, Y.: Effect of accommodation coefficient on thermal creep flow of rarefied gas. Trans. Japan Soc. Aero. Space Sci. 15, 117-123 (1972)

[38] Onishi, Y.: Effects of accommodation coefficient on shear flow of rarefied gas. Trans. Japan Soc. Aero. Space Sci. 17, 93-98 (1974)

[39] Onishi, Y.: Effects of accommodation coefficient on temperature and density fields in a slightly rarefied gas. Trans. Japan Soc. Aero. Space Sci. 17, 151-159 (1974)

[40] Pekeris, C.L., Alterman, Z.: Solution of the Boltzmann-Hilbert integral equation II. the coefficients of viscosity and heat conduction. Proc. Natl. Acad. Sci. 43, 998-1007 (1957)

[41] Pieraccini, S., Puppo, G.: Implicit-explicit schemes for BGK kinetic equations. J. Sci. Comput. 32, 1-28 (2007)

[42] Shakhov, E.M.: Generalization of the Krook kinetic relaxation equation. Fluid Dyn. 3, 95-96 (1968)

[43] Sharipov, F.: Application of the Cercignani-Lampis scattering kernel to calculations of rarefied gas flows. II. Slip and jump coefficients. Eur. J. Mech. B/Fluids 22, 133-143 (2003)

[44] Sharipov, F., Seleznev, V.: Data on internal rarefied gas flows. J. Phys. Chem. Ref. Data 27, 657-706 (1998)

[45] Siewert, C.E.: The linearized Boltzmann equation: Concise and accurate solutions to basic flow problems. Z. angew. Math. Phys. 54, 273-303 (2003)

[46] Siewert, C.E.: The linearized Boltzmann equation: a concise and accurate solution of the temperature-jump problem. J. Quant. Spectr. Rad. Transf. 77, 417-432 (2003) 
[47] Siewert, C.E.: Viscous-slip, thermal-slip, and temperature-jump coefficients as defined by the linearized Boltzmann equation and the Cercignani-Lampis boundary condition. Phys. Fluids 15, 1696-1701 (2003)

[48] Siewert, C.E., Sharipov, F.: Model equations in rarefied gas dynamics: Viscousslip and thermal-slip coefficients. Phys. Fluids 14, 4123-4129 (2002)

[49] Siewert, C.E., Thomas Jr., J.R.: Half-space problems in the kinetic theory of gases. Phys. Fluids 16, 1557-1559 (1973)

[50] Sone, Y.: Kinetic theory analysis of linearized Rayleigh problem. J. Phys. Soc. Jpn. 19, 1463-1473 (1964)

[51] Sone, Y.: Effect of sudden change of wall temperature in rarefied gas. J. Phys. Soc. Jpn. 20, 222-229 (1965)

[52] Sone, Y.: Thermal creep in rarefied gas. J. Phys. Soc. Jpn. 21, 1836-1837 (1966)

[53] Sone, Y.: Asymptotic theory of flow of rarefied gas over a smooth boundary I. In: Trilling, L., Wachman, H.Y. (eds.) Rarefied Gas Dynamics, pp. 243-253. Academic, New York (1969)

[54] Sone, Y.: Asymptotic theory of flow of rarefied gas over a smooth boundary II. In: Dini, D. (ed.) Rarefied Gas Dynamics, Vol. II, pp. 737-749. Editrice Tecnico Scientifica, Pisa (1971)

[55] Sone, Y.: Asymptotic theory of a steady flow of a rarefied gas past bodies for small Knudsen numbers. In: Gatignol, R., Soubbaramayer (eds.) Advances in Kinetic Theory and Continuum Mechanics, pp. 19-31. Springer, Berlin (1991)

[56] Sone, Y.: Kinetic Theory and Fluid Dynamics. Birkhäuser, Boston (2002)

[57] Sone, Y.: Molecular Gas Dynamics: Theory, Techniques, and Applications. Birkhäuser, Boston (2007)

[58] Sone, Y., Aoki, K.: Slightly rarefied gas flow over a specularly reflecting body. Phys. Fluids 20, 571-576 (1977)

[59] Sone, Y., Aoki, K.: Steady gas flows past bodies at small Knudsen numbersBoltzmann and hydrodynamic systems. Transp. Theory Stat. Phys. 16, 189-199 (1987)

[60] Sone, Y., Aoki, K., Takata, S., Sugimoto, H., Bobylev, A.V.: Inappropriateness of the heat-conduction equation for description of a temperature field of a stationary gas in the continuum limit: Examination by asymptotic analysis and numerical computation of the Boltzmann equation. Phys. Fluids 8, 628-638 (1996); Erratum, Phys. Fluids 8, 841 (1996)

[61] Sone, Y., Bardos, C., Golse, F., Sugimoto, H.: Asymptotic theory of the Boltzmann system, for a steady flow of a slightly rarefied gas with a finite Mach number: General theory. Eur. J. Mech. B/Fluids 19, 325-360 (2000) 
[62] Sone, Y., Ohwada, T., Aoki, K.: Temperature jump and Knudsen layer in a rarefied gas over a plane wall: Numerical analysis of the linearized Boltzmann equation for hard-sphere molecules. Phys. Fluids A 1, 363-370 (1989)

[63] Sone, Y., Onishi, Y.: Kinetic theory of evaporation and condensation. J. Phys. Soc. Jpn. 35, 1773-1776 (1973)

[64] Sone, Y., Onishi, Y.: Kinetic theory of evaporation and condensationhydrodynamic equation and slip boundary condition. J. Phys. Soc. Jpn. 44, 19811994 (1978)

[65] Takata, S., Hattori, M.: Asymptotic theory for the time-dependent behavior of a slightly rarefied gas over a smooth solid boundary. J. Stat. Phys. 147, 1182-1215 (2012)

[66] Titarev, V.A.: Conservative numerical methods for model kinetic equations. Computers and Fluids 36, 1446-1459 (2007)

[67] Wakabayashi, M., Ohwada, T., Golse, F.: Numerical analysis of the shear and thermal creep flows of a rarefied gas over the plane wall of a Maxwell-type boundary on the basis of the linearized Boltzmann equation for hard-sphere molecules. Eur. J. Mech. B/Fluids 15, 175-201 (1996)

[68] Welander, P.: On the temperature jump in a rarefied gas. Ark. Fys. 7, 507-553 (1954) 